\title{
Design and implementation of a multithreaded softcore processor with tightly coupled hardware real-time operating system
}

Terance Prabhasara Wijesinghe

West Virginia University

Follow this and additional works at: https://researchrepository.wvu.edu/etd

\footnotetext{
Recommended Citation

Wijesinghe, Terance Prabhasara, "Design and implementation of a multithreaded softcore processor with tightly coupled hardware real-time operating system" (2008). Graduate Theses, Dissertations, and Problem Reports. 2682.

https://researchrepository.wvu.edu/etd/2682

This Thesis is protected by copyright and/or related rights. It has been brought to you by the The Research Repository @ WVU with permission from the rights-holder(s). You are free to use this Thesis in any way that is permitted by the copyright and related rights legislation that applies to your use. For other uses you must obtain permission from the rights-holder(s) directly, unless additional rights are indicated by a Creative Commons license in the record and/ or on the work itself. This Thesis has been accepted for inclusion in WVU Graduate Theses, Dissertations, and Problem Reports collection by an authorized administrator of The Research Repository @ WVU. For more information, please contact researchrepository@mail.wvu.edu.
} 
Design and Implementation of a Multithreaded Softcore Processor with Tightly Coupled Hardware Real-Time Operating System

\author{
Terance Prabhasara Wijesinghe
}

Thesis Submitted to the

College of Engineering and Mineral Resources

at West Virginia University

In partial fulfillment of the requirements

for the degree of

\author{
Master of Science \\ in \\ Electrical Engineering \\ Powsiri Klinkhachorn, Ph.D., Chair \\ Roy S. Nutter, Ph.D. \\ Afzel Noore, Ph.D. \\ David W. Graham, Ph.D.
}

Lane Department of Computer Science and Electrical Engineering

Morgantown, West Virginia

2008

Keywords: Hardware RTOSs, RTOSs, Softcore Processors, Multithreaded

Processors, FPGAs

Copyright 2008 Terance P. Wijesinghe 


\section{Abstract \\ Design and Implementation of a Multithreaded Softcore Processor with Tightly Coupled Hardware Real-Time Operating System}

Terance Prabhasara Wijesinghe

The objective of this thesis is to design and implement an FPGA-based softcore processor with hardware Real Time Operating System (RTOS).

RTOSs provide an abstracted environment on top of the hardware with different mechanisms to simplify and coordinate the behavior of the system. Software mechanisms including multithreading, semaphores, timers, interrupts, etc. consume large amounts of CPU time which increases overhead and contributes to the degradation of the performance. In order to offload this overhead, RTOSs with hardware acceleration have been a research topic with considerable interest. Most research works are focused on the design of a hardware RTOS external to the CPU where CPU interacts with the hardware RTOS module as a memory mapped device. This approach provides the flexibility of using the general purpose processor, however, fast context switching is not possible unless a special CPU with a duplicated register file is equipped. To this end, tightly coupling the RTOS functionality to the CPU pipeline is a good candidate for further investigation. Multithreaded processors are a distinct area of research focusing on reducing the pipeline hazards by interleaving instructions from different threads. Most of the multithreading techniques support fast context switching mechanisms in order to support cycle interleaving of instructions. The fast context switching ability inherent to multithreaded processors can be used to implement OS level multithreading and low latency interrupt service threads. This thesis investigates on the possibilities of merging previous research work on hardware RTOSs and multithreaded processors to obtain an overall improvement. Attachment of a hardware RTOS to the pipeline increases the determinism by eliminating external interfaces with nondeterministic bus arbitration schemes and reduces the logic required to implement the external interfaces.

The implemented prototype processor is instruction set compatible with Xilinx Microblaze and has a five stage multithreaded pipeline. The pipeline is extended with a static priority preemptive scheduler, semaphore and timer modules. The instruction set is augmented with several instructions to support thread creation, deletion, synchronization and time management. Results suggest that RTBlaze can completely remove the OS overhead at the cost of doubled processor size. Zero overhead scheduler, context switching, thread synchronization, timers and five-cycle interrupt latency give excellent performance as well as the increased determinism compared with software RTOSs. 


\section{Acknowledgements}

I would first like to thank my advisor Dr. Powsiri Klinkhachorn for the support and guidance he has given me during my two years as a graduate student. I am also grateful to Dr. David W. Graham, Dr. Roy S. Nutter and Dr. Afzel Noore for their support.

I take this opportunity to thank my parents and sister for supporting and guiding me in my decisions. I am also thankful to all those who helped me directly or indirectly in completion of this work.

Prabhasara Wijesinghe 


\section{Contents}

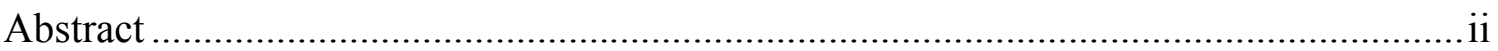

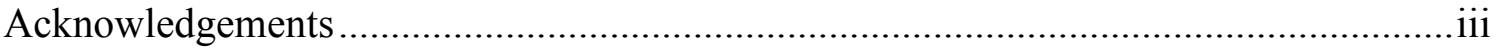

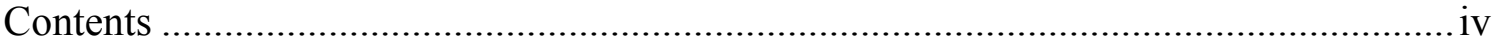

List of Figures and Illustrations .............................................................................. vi

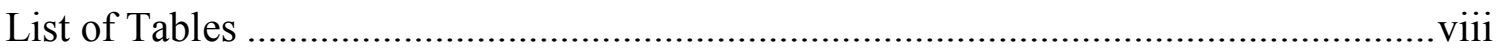

List of Symbols, Abbreviations and Nomenclature .......................................................ix

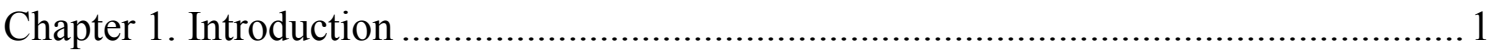

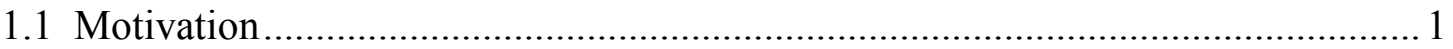

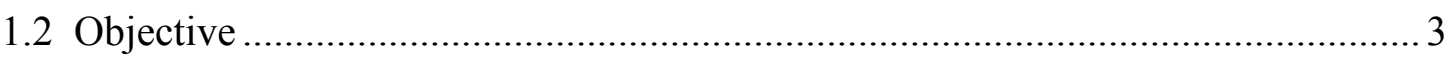

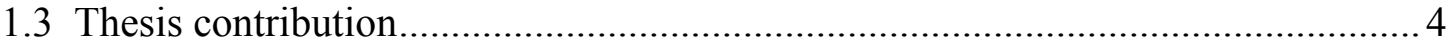

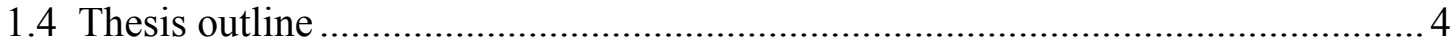

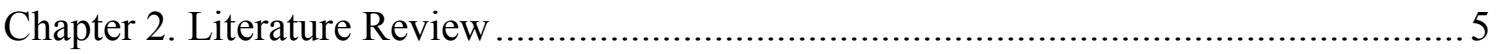

2.1 Field Programmable Gate Arrays ………………....................................... 5

2.2 Platform FPGAs - Vertex II Pro ……………................................................. 8

2.3 Systems on a Programmable Chip (SoPC) Design ............................................... 10

2.4 Configurable Softcore Processors for FPGAs ………………........................... 14

2.5 Real-time Operating Systems for SoPC Based Systems ...................................... 15

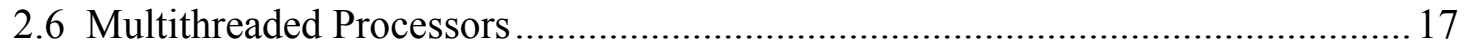

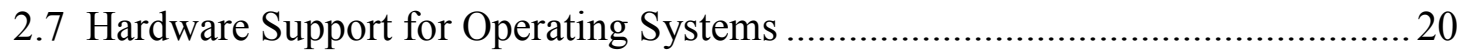

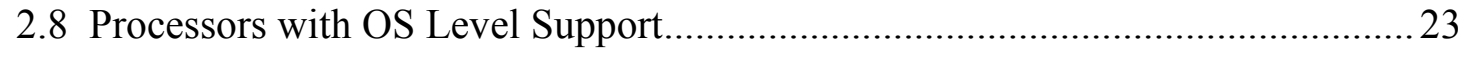


Chapter 3. Design and Implementation of the RTBlaze

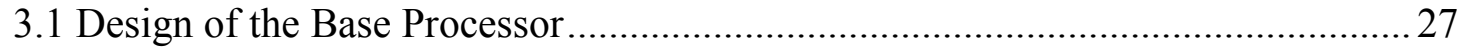

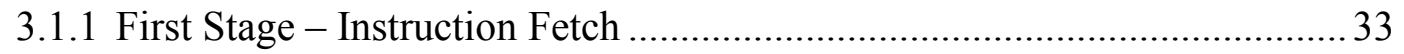

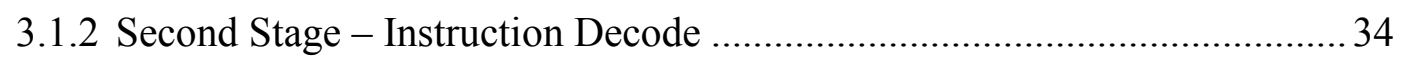

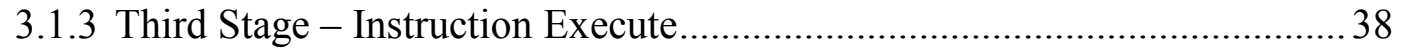

3.1.4 Fourth Stage - Data Memory Access .............................................. 40

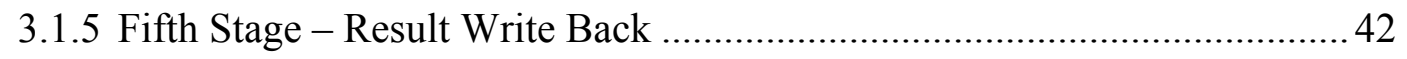

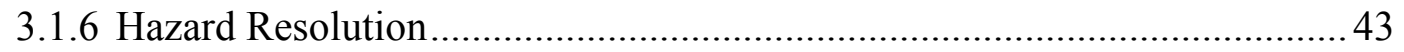

3.2 Architecture of RTOS ............................................................................ 46

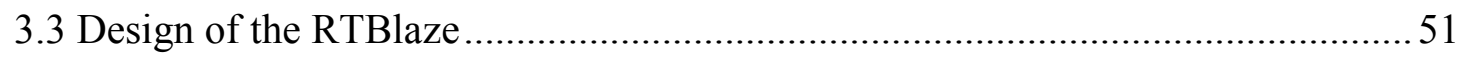

3.3.1 The Fetch Stage with Scheduling Support ..........................................56

3.3.2 Support for Interrupt Service Threads ....................................................59

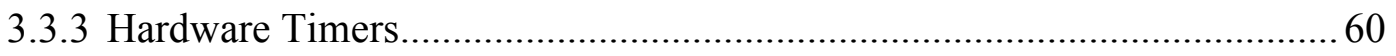

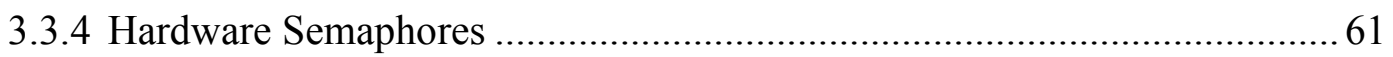

3.4 Implementation and Performance of the RTBlaze........................................... 64

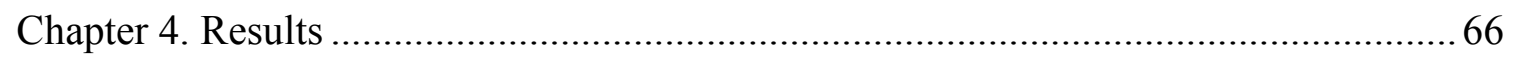

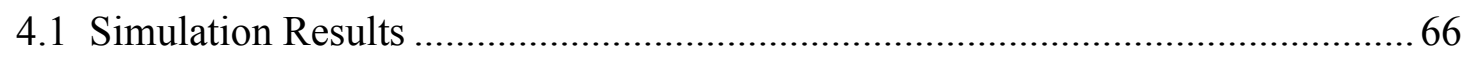

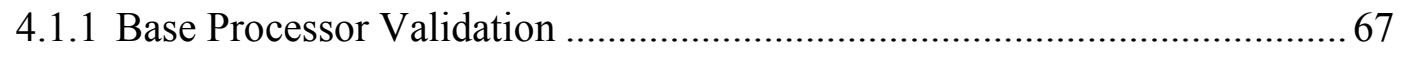

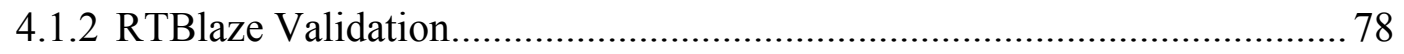

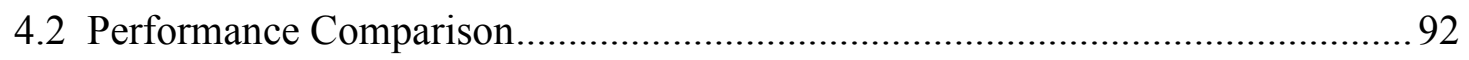

4.2.1 Comparison of the Performance of RTBlaze and Software RTOSs ............93

4.3 Size of the Kernel Footprint ....................................................................... 94

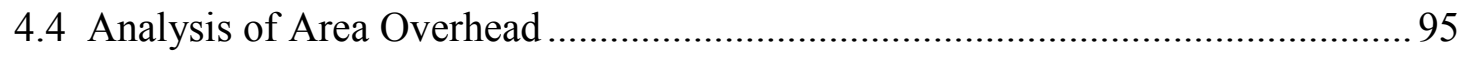

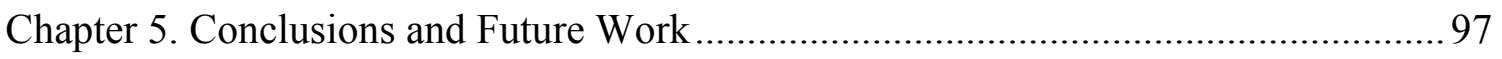

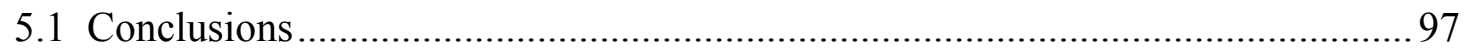

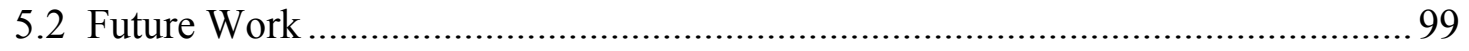

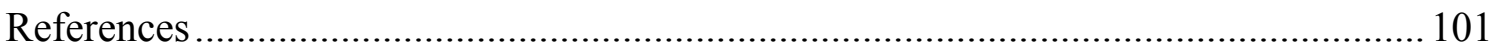




\section{List of Figures and Illustrations}

Figure 2.1: Simplified Internal Architecture of an FPGA ............................................ 7

Figure 2.2: Generic Logic Element ........................................................................ 7

Figure 2.3: Internal Architecture of Vertex II Pro Platform FPGA [16] ......................... 9

Figure 2.4: Local Interconnects of the Slices in a Single CLB [16] ............................... 10

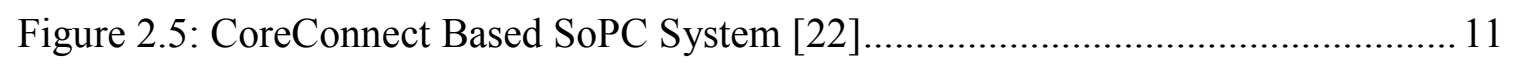

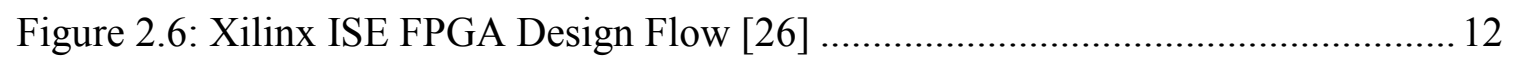

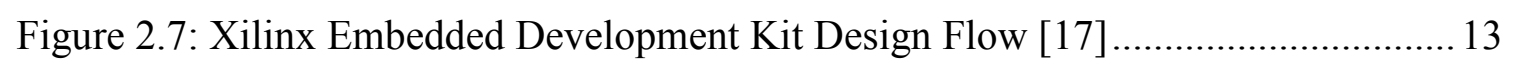

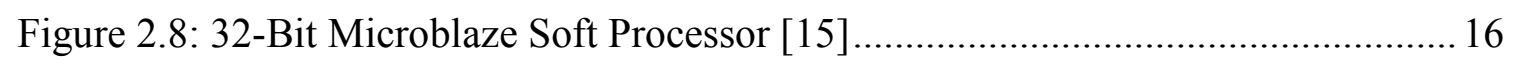

Figure 2.9: Pipeline Hazards Due to Data Dependencies ........................................... 18

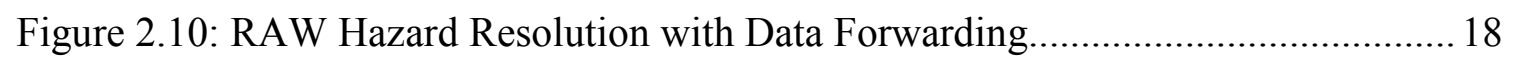

Figure 3.1: EDK Integration of the Base Processor..................................................... 28

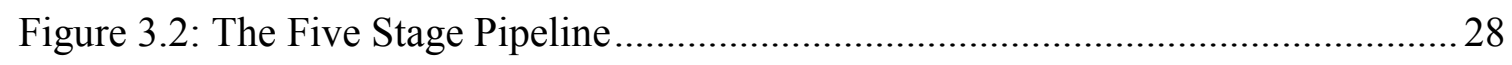

Figure 3.3: Block Diagram of the Proposed Base Processor ...................................... 29

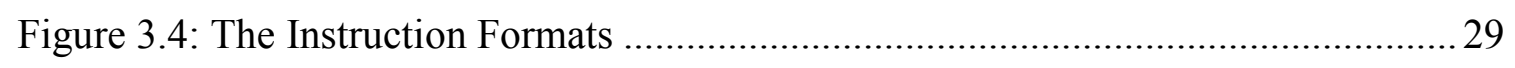

Figure 3.5: Simplified Five Stage Pipeline of the Base Processor.................................. 31

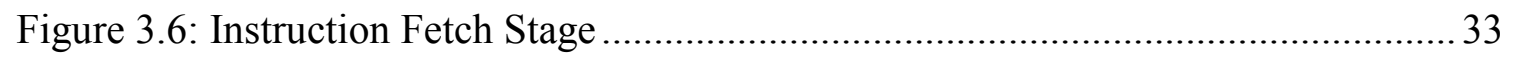

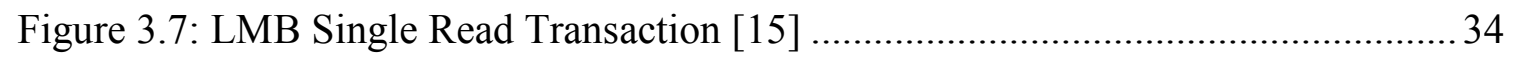

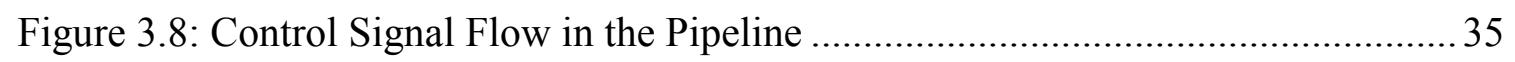

Figure 3.9: Register File with Three Read Ports and One Write Port ............................. 37

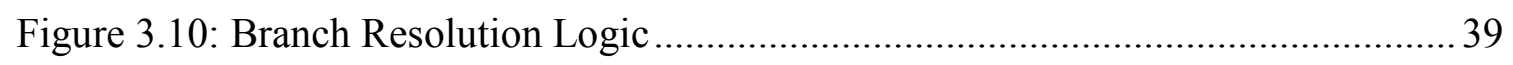

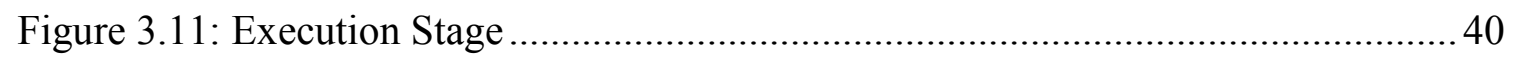

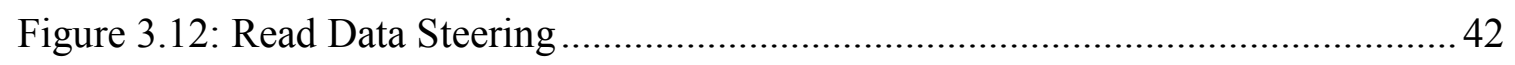

Figure 3.13: Base Processor Pipeline with Hazard Control.......................................... 45

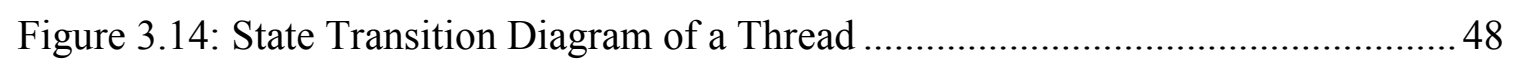

Figure 3.15: Simplified Architecture of a RTOS Kernel Running On a SOPC.............. 49

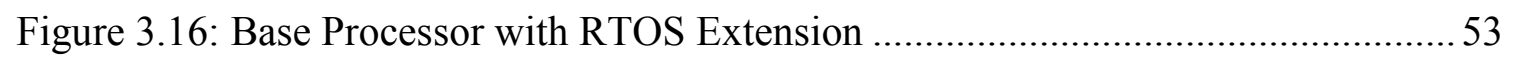

Figure 3.17: State Transition Diagram of an RTBlaze Thread ......................................54

Figure 3.18: Multithread Pipeline with Scheduling Support ...................................... 55

Figure 3.19: Microarchitecture of the Static Priority Based Thread Scheduler ...............57 
Figure 3.20: Modified Prefetch Stage with Scheduling Support ...................................58

Figure 3.21: Extension of the Scheduler to Support Hardware ISTs............................59

Figure 3.22: Direct Mapped Hardware Timers ....................................................... 61

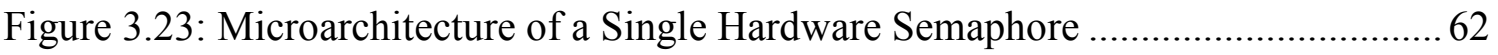

Figure 3.24: Microarchitecture of a Single Priority Queue Element ............................. 64

Figure 4.1: Sample Program- Simulation Results (80ns to 190ns) Part I....................... 68

Figure 4.2: Sample Program - Simulation Results (80ns to 190ns) Part II...................... 69

Figure 4.3: Sample Program - Simulation Results (180ns to 290ns) Part I.................... 70

Figure 4.4: Sample Program - Simulation Results (180ns to 290ns) Part II................... 71

Figure 4.5: Load/Store Instructions - Simulation Results (30ns To 130ns) Part I.......... 75

Figure 4.6: Load/Store Instructions - Simulation Results (30ns to 130ns) Part II........... 76

Figure 4.7: Simulation Results and Pipeline Events - Thread Creation.......................... 79

Figure 4.8: Execution of Sleep Instruction - Thread 2 Sleeps for Five Cycles .............. 81

Figure 4.9: Simulation Results and Pipeline Events - Creation of a New IST ..............85

Figure 4.10: Simulation Results and Pipeline Events - External Interrupt ....................86

Figure 4.11: Simulation Results and Pipeline Events - Interrupt Clear ......................... 87

Figure 4.12: Semaphore Instructions - Simulation Results (580ns to 680ns) Part I....... 89

Figure 4.13: Semaphore Instructions - Simulation Results (680ns to 780ns) Part II...... 90 


\section{List of Tables}

Table 3.1: A Partial List of Microblaze Instruction Set .................................................. 30

Table 3.2: Flow of Control Signals through Each Pipeline Stage ................................... 36

Table 3.3: Operations Supported by the ALU .................................................................... 38

Table 3.4: Conditional Control Transfer Instructions ........................................................ 39

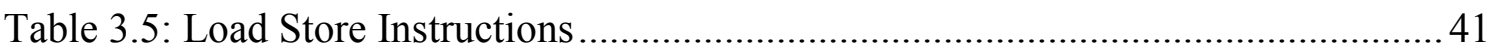

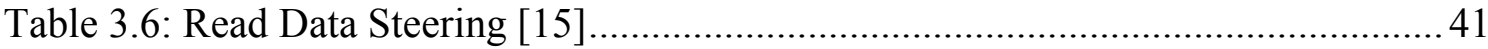

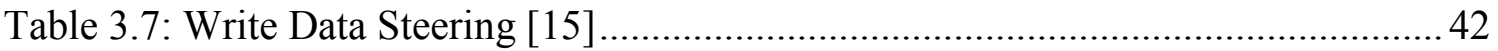

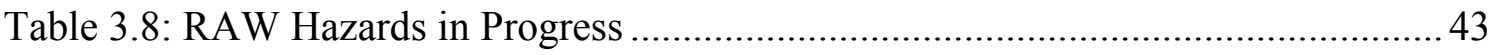

Table 3.9: Hazard Resolution........................................................................................ 44

Table 3.10: Basic System Calls Provided by a RTOS .................................................... 46

Table 3.11: RTBlaze Instructions for RTOS Services ...................................................52

Table 4.1: Assembly Program Used to Validate Arithmetic and Branch Instructions ....67

Table 4.2: Execution of the Program - Pipeline Events during the First Iteration........... 72

Table 4.3: Execution of the Program - Pipeline Events during the Last Iteration .......... 73

Table 4.4: Program to Validate Data Memory Access ..................................................... 74

Table 4.5: Execution of Load $/$ Store Instructions- Pipeline Events ................................ 77

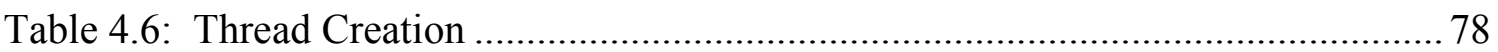

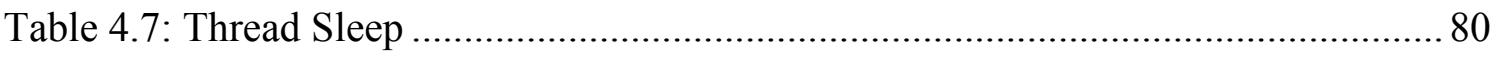

Table 4.8: Execution of Sleep Instruction - Pipeline Events.......................................... 82

Table 4.9: A Program to Validate the IST Mechanism.................................................... 84

Table 4.10: A Program to Validate Semaphore Operations............................................... 88

Table 4.11: Execution of Semaphore Instructions- Pipeline Events ................................ 91

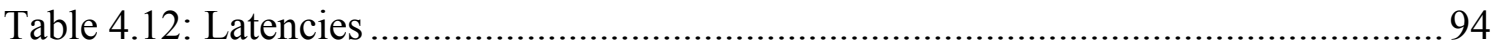

Table 4.13: Kernel Footprint Comparison ............................................................. 94

Table 4.14: Resource Utilization Of RTOS Extensions ................................................. 95

Table 4.15: Breakdown of Area Overhead Due to RTOS Extensions ............................. 96 


\section{List of Symbols, Abbreviations and Nomenclature}

\begin{tabular}{ll} 
FPGA & Field Programmable Gate Array \\
SOPC & System on a Programmable Chip \\
ADL & Architectural Definition Language \\
VHDL & Very high speed Hardware Description Language \\
PLB & Processor Local Bus \\
OPB & On Chip Peripheral Bus \\
IP & Intellectual Property \\
SoC & System on a Chip \\
RTOS & Real-time Operating System \\
OS & Operating System \\
LVTTL & Low Voltage Transistor Transistor Logic \\
LVCMOS & Low Voltage Complementary Metal Oxide \\
CLB & Semiconductor \\
FSL & Configurable Logic Block \\
LUT & Fast Simplex Link \\
JTAG & Look Up Table \\
EDF & Joint Test Action Group \\
LMB & Earliest deadline First \\
TSS & Local Memory Bus \\
IST & Task State Segment \\
GCC & Interrupt Service Thread \\
TCB & GNU Compiler Collection \\
DCR & Task Control Block \\
& Device Control Register \\
\hline
\end{tabular}




\section{Chapter 1}

\section{Introduction}

\subsection{Motivation}

Embedded systems have become omnipresent, and most of them have real-time behavior. Unlike general purpose systems, deadlines are not relaxed and determinism is a very important aspect. These systems are supposed to meet given deadlines and a missed deadline may even be catastrophic.

For the high performance hard real-time systems the overhead of operating system cannot be neglected. The Operating System (OS) scheduler and context switching overhead collectively consume the useful CPU time. Interrupt processing overhead contributes to the interrupt latency and in turn reduces the system response time. In order to offload these overheads, real-time operating systems with hardware acceleration have been a research topic with considerable interest. 
Before the Field Programmable Gate Array (FPGA) based configurable embedded systems enter the mainstream, all of the research work on hardware-based acceleration of OSs are focused on silicon implementations [1-4]. To support OS primitives at the hardware level, several research groups have made efforts to implement OSs on silicon. These are separate chips or Intellectual Property (IP) cores connected to the external CPU bus as memory mapped peripherals enabling the use of general purpose CPUs. Most research groups have focused on only the scheduler component design in hardware, since most real-time systems demand complicated schedulers and most of those algorithms have the property of paralizability where the hardware implementation is the ideal solution [3, 5-7]. The external chip based hardware scheduler performs the timekeeping and the scheduling and raises an interrupt to the CPU in case preemption is required. Upon interruption, the $\mathrm{CPU}$ communicates with the hardware scheduler, obtains the next thread ID, and performs a context switch. This method off-loads the kernel timer tick counting and execution of the scheduler. Not only the scheduler, but also RTOS functionality such as timers and semaphores are excellent candidates for hardware migration [1, 4]. All the previous researchers have proven that the hardware implementations give promising results over the software-based conventional RTOS implementations.

Intellectual Property (IP) or separate chip based approach gives the flexibility of using a general purpose processor, but determinism is again sacrificed due to nondeterministic bus arbitration schemes equipped in Systems on a Programmable Chip (SoPC) architectures. Round robin bus arbitration makes it feasible to have a deterministic way of interacting with IP services, but it degrades the overall system performance. One way to overcome this issue is to attach the IP as a coprocessor with special bus interface [8]; however, these methods are not capable of removing the context switching overhead. In order to remove the overhead due to context switching, a special CPU has to be used with a dedicated interface with the hardware scheduler [9]. One method is to use a register file with a transparent loading mechanism of the context of the thread in the background. To this end, tightly coupling the RTOS functionality to the processor itself is a good candidate for further investigation. 
Multithreaded processors are a distinct area of research focusing on reducing the pipeline hazards by interleaving instructions from different threads [10]. Multithreading comes in three different flavors: cycle interleaved, block interleaved, and hybrid. Cycle interleaved multithreaded processors inherit a special feature of switching the thread contexts in a single cycle. This interesting feature can be used to eliminate the overhead due to context switching in the OS domain. There are some research proposals to utilize this fast context switching ability to implement fast interrupt service threads [11-14].

This thesis investigates on the possibilities of merging these two orthogonally investigated concepts of multithreaded processors and hardwired RTOS implementations to obtain an overall improvement. Multithreaded pipeline provides zero overhead context switching as well as low latency interrupt handling mechanism, while the hardware migrated RTOS functionality provides low overhead thread, timer and semaphore manipulation. Attachment of hardware RTOS to the pipeline increases the determinism by eliminating external interfaces where the arbitration is an issue.

\subsection{Objective}

The objective of this thesis is to design and implement a prototype 32 bit RISC multithreaded processor, RTBlaze, with tightly coupled hardware RTOS attached to its pipeline. The first phase of this thesis designs a base processor instruction set compatible with Xilinx MicroBlaze [15].

The second phase of this thesis extends the base processor with hardware RTOS support. The base processor pipeline is extended to support cycle and block interleaved multithreading. A special thread scheduler in the fetch stage decides the next ready thread based on a preemptive, static priority based scheduling algorithm realized in hardware. The main goal behind making the processor multithreading ready is to accommodate a fast context switching method so the thread scheduler can simply schedule any thread in any cycle provided that thread is in the ready state. This technique enables fast interrupts service threads where the interrupt latency is only the depth of the pipeline. The processor also implements timer queues and semaphore queues tightly coupled to the pipeline. These help to achieve zero overhead OS level 
functionality and excellent determinism. The instruction set of the base processor is extended with new instructions in order to access above mentioned RTOS services.

The proposed processor is implemented using synthesizable Very high speed Hardware Definition Language (VHDL) code targeting Vertex II architecture [16]. Structural level modeling of VHDL is used in order to align the multithreaded architecture with the logic resources of the Vertex II whenever necessary. The processor can be integrated with the Xilinx tool chain [17], and the GNU compiler can be used to assemble C program code.

\subsection{Thesis contribution}

Following contributions are made by this thesis,

1. Design of a softcore processor compatible with Xilinx Microblaze optimized for Vertex II architecture.

2. Design of a FPGA optimized multithreaded processor with a tightly coupled hardware RTOS.

\subsection{Thesis outline}

Chapter 2 provides a brief background on the tools and subject material of this research by introducing the concepts of modern embedded systems design based on SoPCs, operating systems, and multithreaded processors. This chapter also provides a brief survey of previous research efforts on hardware implementations of RTOSs. Chapter 3 describes the design and implementation of the RTBlaze processor. The chapter starts with a detailed discussion of the MicroBlaze compatible 32-bit base processor design. The chapter extends the discussion into the architectural modifications made to the base processor in order to augment the instruction set with hardware RTOS support. Chapter 4 presents the validation and the simulation results of the base processor and the RTBlaze processor and compares the performance of the RTBlaze with some off-the-shelf RTOSs. The chapter continues by investigating the area overhead that occurs due to the RTOS extensions of the Instruction Set Architecture (ISA). The research is summarized along with a postulation of future work in Chapter 5. 


\section{Chapter 2}

\section{Literature Review}

The first part of this chapter summarizes the technical background related to the state of the art embedded systems design based on FPGAs. The second part discusses the foundation of the thesis which involves the current research work related to the hardware implemented operating systems and processors with OS level support.

\subsection{Field Programmable Gate Arrays}

Traditionally, FPGA technology was targeted for glue logic replacement of complex digital systems and replacement of complex state machines. As the technology evolved over the time, current FPGA technology has become advanced enough to implement complex Multiprocessor System On a Chip (MPSoC) [18] solutions targeting high end embedded applications [19]. Minimum time to market and the 
possibility of making design changes at later stages of the design flow have made FPGA based SoCs a natural selection for low to medium volume embedded system products.

FPGAs represent a family of fine grained logic architectures and contain hundreds of prebuilt logic elements immersed on a programmable routing resource. Apart from the logic elements and routing resources, modern FPGAs contain various specialized silicon immersed blocks ranging from memories to hard processor cores. These resources can be configured to implement any custom hardware design using the FPGA vendor's design tool chain. These tool chains use state of the art placing and routing algorithms to map a given hardware design into the targeted FPGA architecture. Synchronous Hardware Description Languages (HDLs) such as VHDL and Verilog HDL are used as the main method of design entry. Given an HDL design, the tool chain can generate a bit stream file that can be used to configure the targeted FPGA. FPGA vendors further extend their tool chains from logic design to the embedded space by providing various tool chains.

Figure 2.1 illustrates a simplified view of the internal architecture of a generic FPGA where a sea of logic elements is immersed in a configurable routing resource. Logic elements are the basic building blocks of any FPGA architecture. The basic logic element as shown in Figure 2.2 consists of a Look Up Table (LUT), a flip-flop and a multiplexer. Based on this generic architecture, different FPGA vendors enhance logic elements by adding more configurable features. Those improvements enhance performance and the synthesizability of a given synchronous circuit with minimum resource usage. 


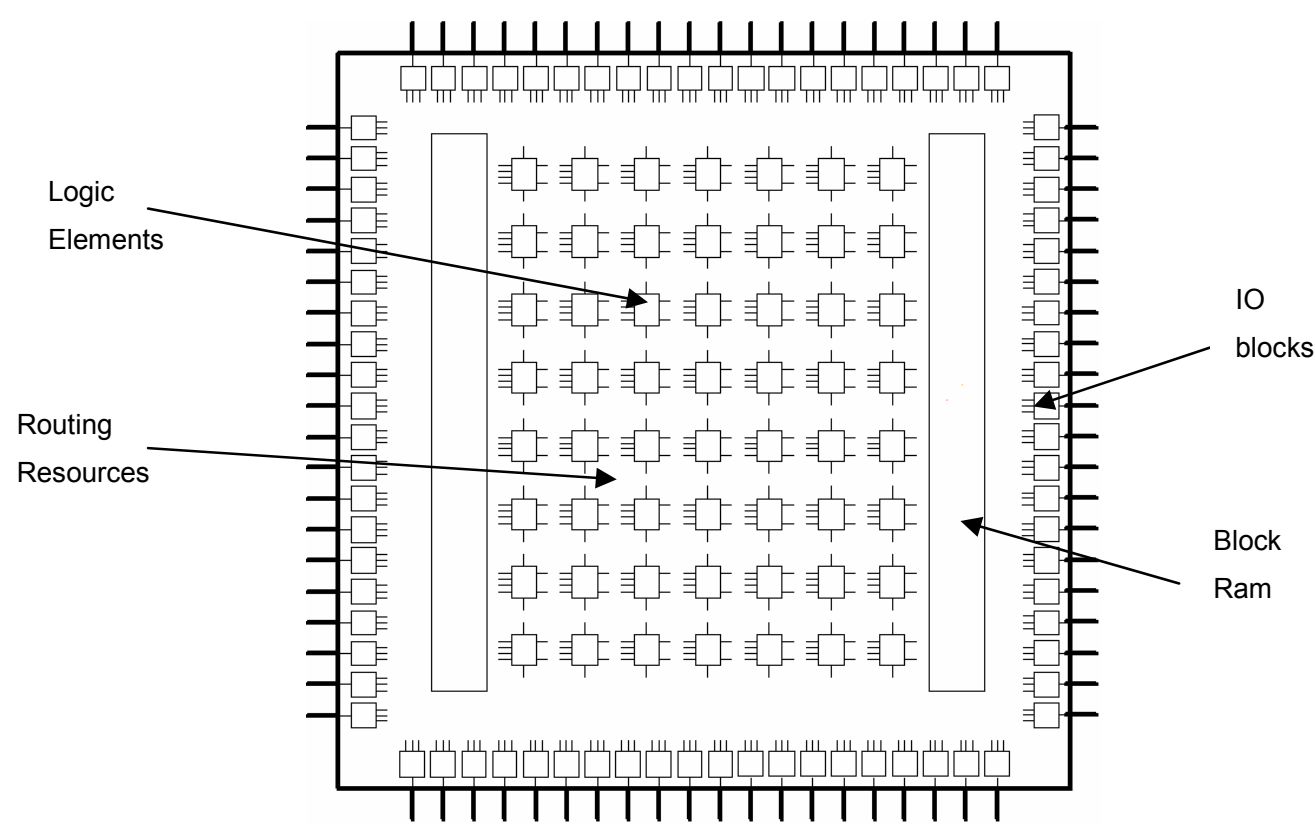

Figure 2.1: Simplified Internal Architecture of an FPGA

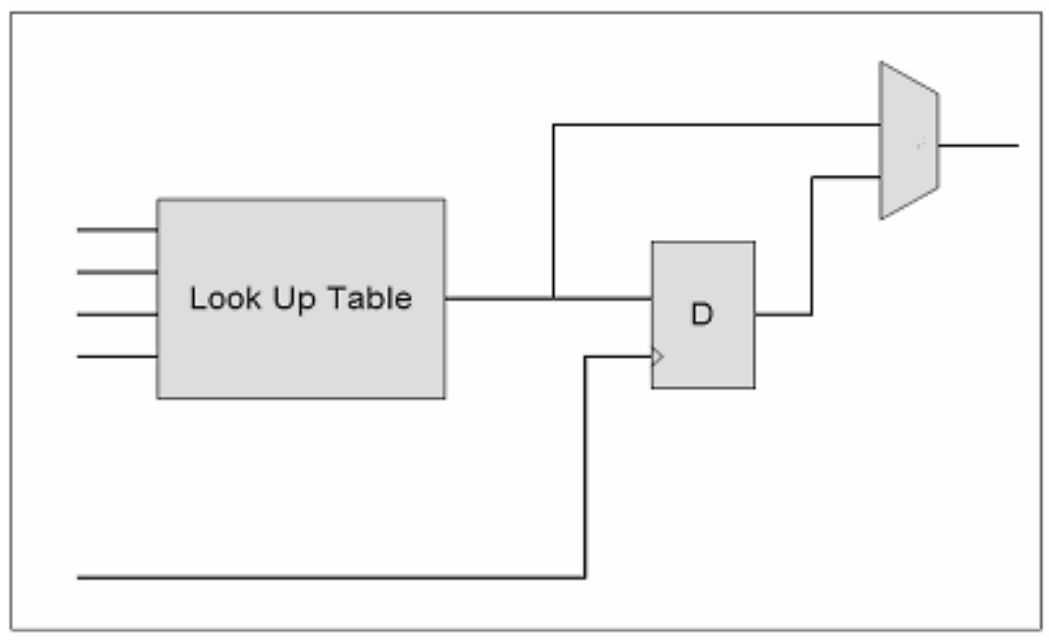

Figure 2.2: Generic Logic Element

The internal routing architecture enables each logic element to be interconnected. These configurable routing resources are usually partitioned into global and local routing networks in order to optimize the interconnections and to reduce the interconnect delays. The output pins of the FPGAs are connected to the routing resources through the configurable IO blocks. These blocks support different IO standards such as LVTTL and LVCMOS in order to support various board level interfaces. 
Routing lines are implemented using MOS transmission gates with SRAM bits controlling the gates. The configurability of the logic elements as well as IO blocks are implemented in a similar fashion using SRAM bits. These SRAM bits are called the configuration memory of the FPGA. In order to implement a custom circuit, the configuration memory of the FPGA needs to be programmed. Most of the FPGAs are

SRAM based and come with JTAG interface or a proprietary interface for the configuration. The design tool chains provided by the vendor have support for FPGA configuration using a special interface cable.

\subsection{Platform FPGAs - Vertex II Pro}

As the density of given technology grows, FPGA vendors tend to integrate dedicated functional blocks into the logic fabric. For example, Xilinx Vertex [3] family has hardcore PowerPC processors directly immersed in the logic fabric capable of running at very high clock speeds. Apart from this hardcore processor trend, most FPGA vendors integrate specialized IO processing blocks around the logic fabric including physical MAC layers, high speed transceivers, etc. These types of FPGAs with specialized silicon immersed IPs are known as platform FPGAs.

Vertex II Pro [16] is a platform FPGA that comes with two PowerPC processor blocks, an array of memory blocks and multipliers immersed in the configurable logic fabric. Vertex family FPGAs target high performance embedded systems and the architecture is tailored specifically for the embedded domain. Figure 2.3 illustrates the internal resources of the FPGA. 


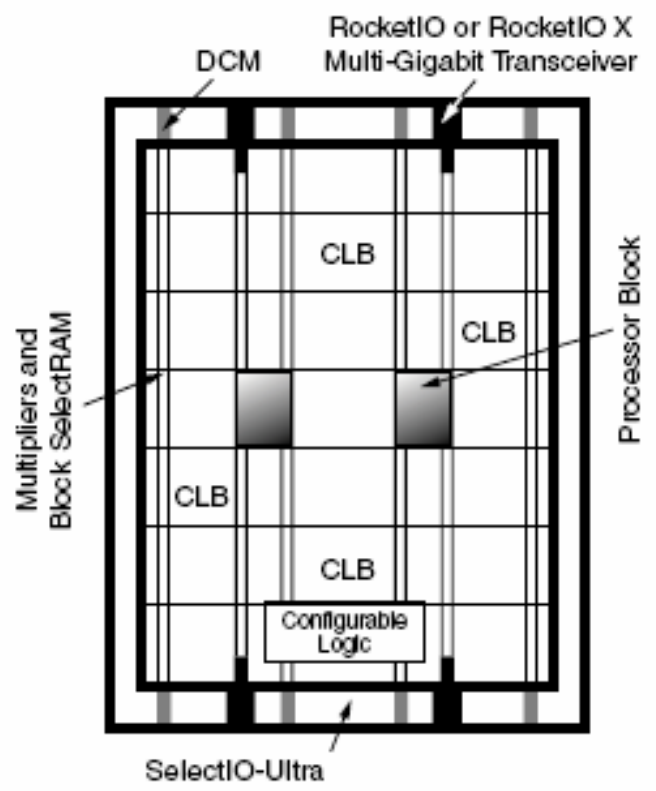

Figure 2.3: Internal Architecture of Vertex II Pro Platform FPGA [16]

Vertex architecture contains a large array of Configurable Logic Blocks (CLBs). Each CLB contains four logic slices. The logic slice is based on the generic logic element explained in Section 2.1 with several enhancements. Two function generators ( $F$ \& G), two storage elements, arithmetic logic gates, large multiplexers, wide function capability, fast carry look-ahead chain and horizontal cascade chain (OR gate) are some noticeable enhancements over the generic logic element. The function generator is based on SRAM and can be configured as a 4-input LUT, or as a 16-bit shift register, or as a 16-bit distributed SelectRAM+ [16] memory. The outputs of these function generators can be optionally routed through two storage elements which are configurable as edgetriggered D-type flip flops or level sensitive latches. Four of those slices are connected together to make a single CLB as shown in Figure 2.4. The slices in the CLB have fast internal interconnects as well as carry and shift chains. Each slice has a dedicated connection to the switching matrix as shown in Figure 2.4 in order to access the global and local interconnect resources. 


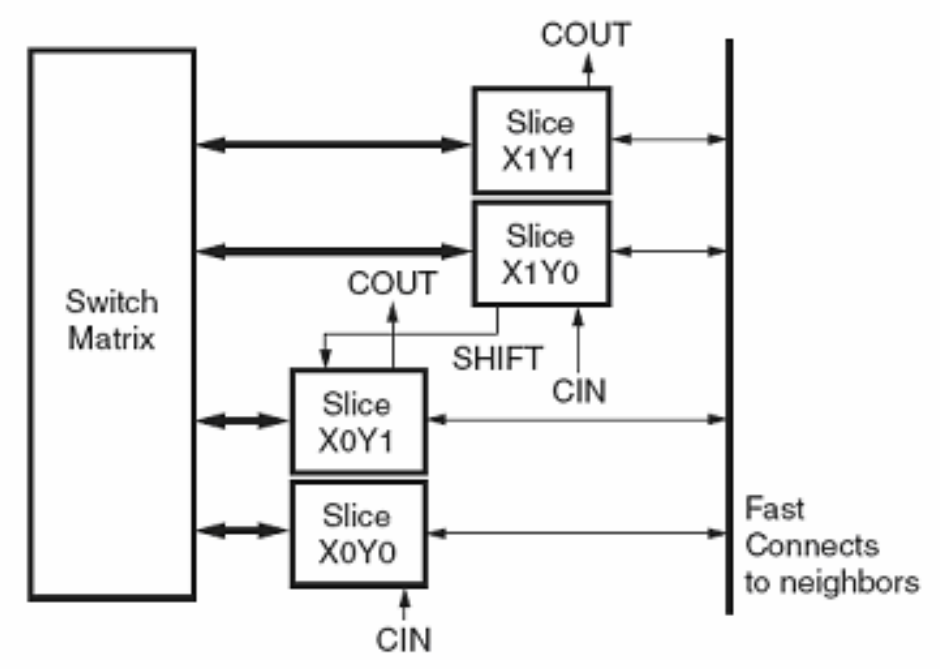

Figure 2.4: Local Interconnects of the Slices in a Single CLB [16]

\subsection{Systems on a Programmable Chip (SoPC) Design}

Traditionally, embedded system designs consist of many different phases. These systems are collections of peripherals connected to a processor through a system level external bus at the PCB level. As the silicon integration evolved over the years, the board level embedded systems design became obsolete, and single chip solutions or SoC have been introduced. These SoC solutions have soon become omnipresent in most embedded systems. The tight coupling of the CPU with its surrounding peripherals at silicon level addresses most signal integrity issues prevalent in board level designs. For example, Intel XScale [20] SoC consists of lots of peripherals with an ARM processor core on a single die. It is optimized for mobile and embedded applications. Internally it uses the AMBA [21] bus standard to glue the peripherals and memory controllers to the ARM core.

SoCs can be fabricated by several technologies such as full custom, standard cell, and using FPGAs. Using the full custom methodology, the fastest operation can be achieved at the cost of increased design time. In full custom design, the entire $\mathrm{SoC}$ is designed at very low level, i.e. using transistors and wires with the assistance of VLSI design tool chains. FPGA based realization is the most flexible method of SoC design which is called SoPC systems. Because of this flexibility inherent to the FPGAs, design 
errors can be easily corrected during the verification process. When it comes to standard cell or full custom methods (ASIC based), correction of design faults has very high prices, because the system is implemented directly on the silicon, which cannot be altered.

When it comes to the SoPC systems, the internal bus architecture is one of the most important aspects. There are several industry standard bus architectures including, AMBA [21], IBM CoreConnect [22] and Wishbone [23]. Xilinx uses IBM's CoreConnect bus standard as system level interconnect architecture. Figure 2.5 illustrates a SoPC design based on CoreConnect bus architecture.

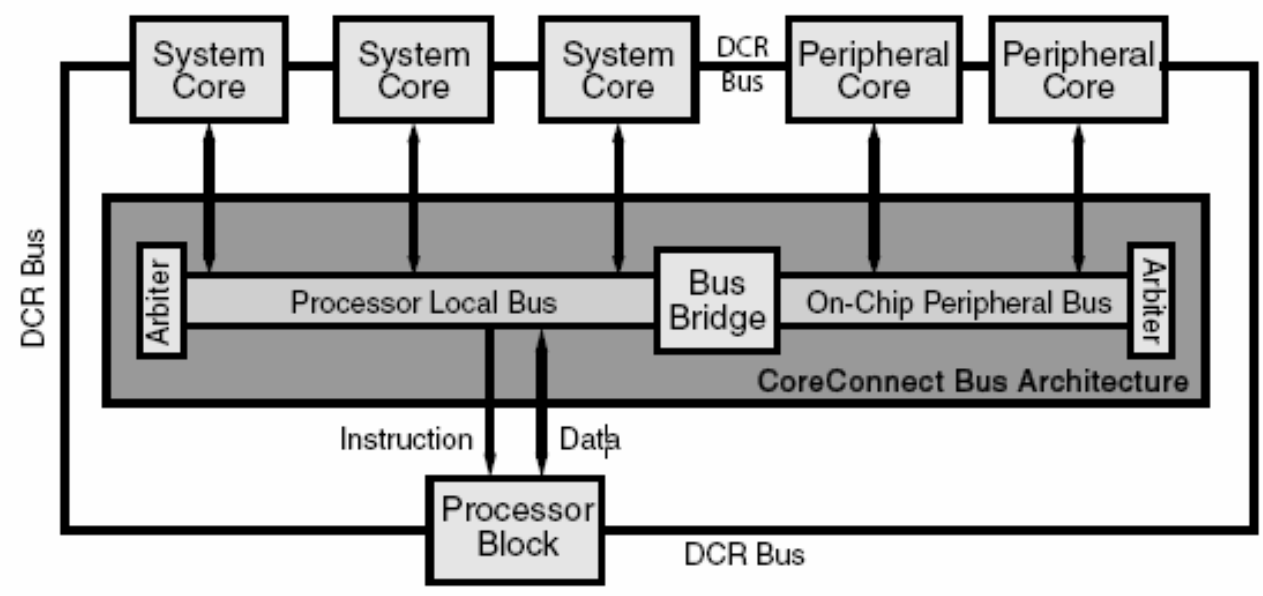

Figure 2.5: CoreConnect Based SoPC System [22]

CoreConnect specification specifies three bus standards for IP interconnect. Processor Local Bus (PLB) [24] is a high performance bus used for the processor's instruction and data side interfaces. PLB is a high performance, synchronous, centrally arbitrated, spilt transaction bus which comes with different widths, 32-, 64-, and 128-bit. The On-chip Peripheral Bus (OPB) [25] is a medium performance bus used to connect IP cores to the processor. It is also a synchronous and centrally arbitrated bus system. Device Configuration Register (DCR) bus is used to offload the device configuration traffic from the main systems buses. DCR is a low performance bus.

Xilinx uses these standards to define IP level interconnect on the FPGA fabric. The user IP cores need to comply with these interface standards, and the design tool 
chain comes with various IP cores for standard interfaces. These IPs include OPB DRAM controllers, OPB RS232 IPs, OPB SPI IPs, etc.

The tool chain provided by the FPGA vendors greatly simplifies the FPGA design. Xilinx Embedded Development Kit (EDK) [17] provides a graphical interface for high level design of the embedded system while Xilinx Integrated Software Environment (ISE) [26] provides the logic design and simulation environment.

The ISE is the synthesis engine which ultimately maps a given VHDL design to a configuration bit stream for a targeted FPGA. Timing analysis is used at all possible levels of design flow [27] in order to isolate the timing issues at the earliest possible stage as shown in Figure 2.6.

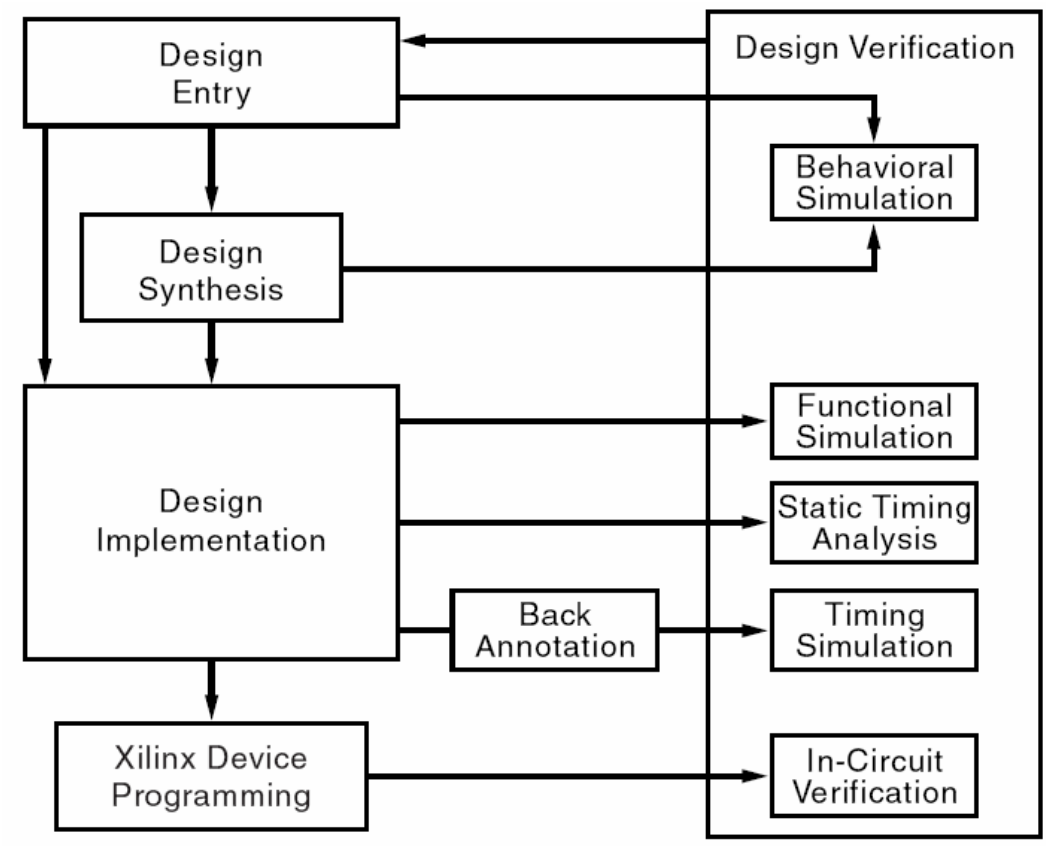

Figure 2.6: Xilinx ISE FPGA Design Flow [26]

Ever increasing design complexity of embedded systems has resulted in a new design philosophy; the codesign [28] of software and hardware. This allows one to reduce the time to market window and accelerate the design process by concurrently designing both hardware and software for the embedded system. Xilinx EDK is an embedded system codesign environment that works on top of ISE. EDK is basically IP interconnect and configuration utility which greatly simplifies the SoPC design. EDK 
integrates the GNU compiler tool chain for cross compilation and various IP cores from Xilinx including a JTAG based debug module [29]. The design flow is illustrated in Figure 2.7.

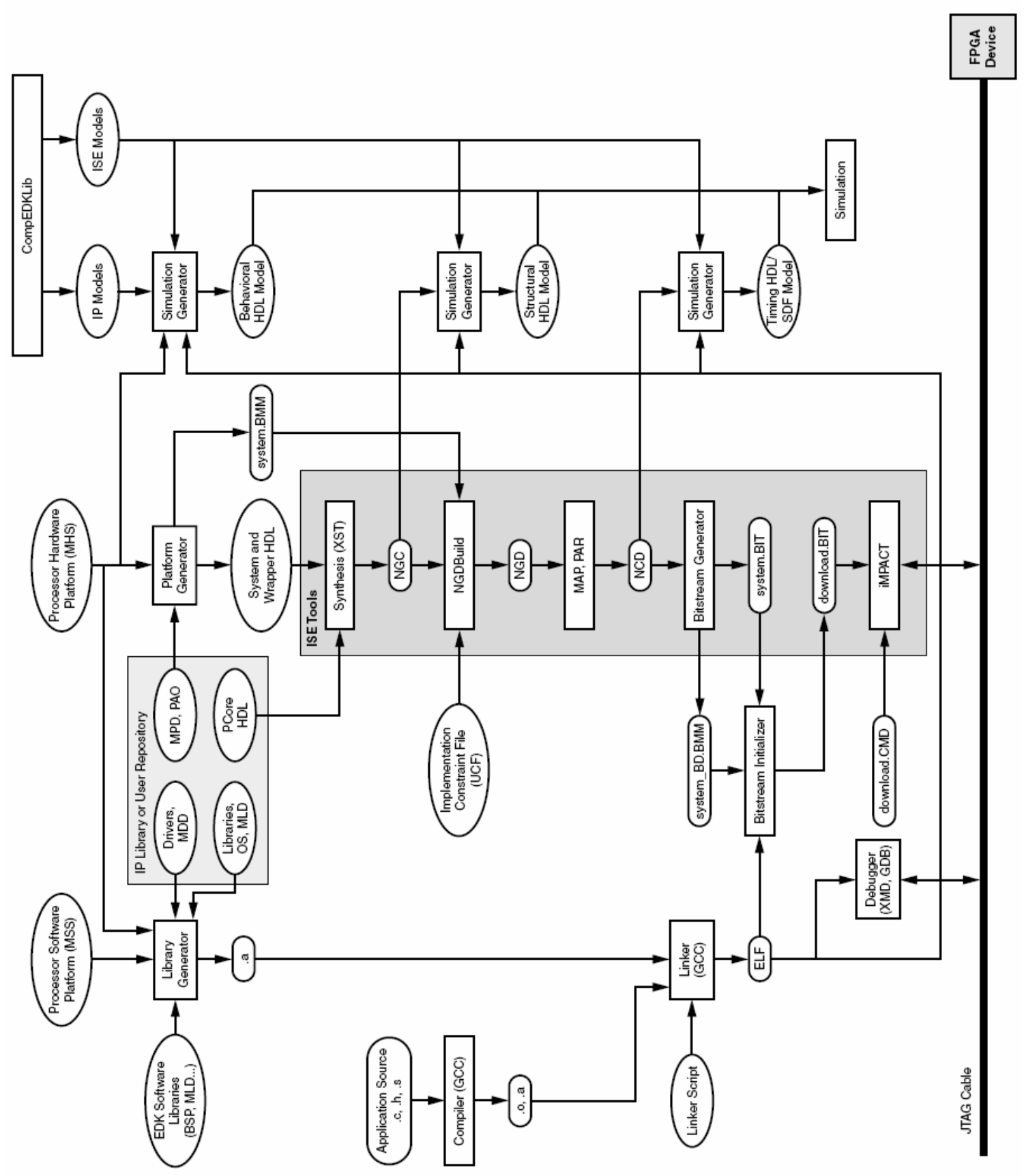

Figure 2.7: Xilinx Embedded Development Kit Design Flow [17] 


\subsection{Configurable Softcore Processors for FPGAs}

Most of the time SoPCs are built using softcore processors. A softcore processor is an IP core fully described using an HDL which can be synthesized in an FPGA. Configurability of a FPGA based SoPC is the most noticeable attractiveness over silicon implemented SoCs. The configurability comes in two types [30, 31]. The dynamic reconfigurablity allows one to reconfigure the full or a portion of the FPGA on the fly. This concept is further extended to provide virtual hardware closely resembling the concept of virtual memory systems used in modern CPUs. Static configurability is the feature which allows the configuration of components at design time. For example, the features of a soft core processor such as number of pipeline stages, use of hardware multipliers, size of the cache memory, etc. can be configured at design time in order to maximally align the architecture to the targeted application domain.

FPGA vendors also offer configurable softcore processor solutions which can be tailored for the specific need of a particular embedded system. These softcore processors are mainly targeted for their own FPGA architectures and optimized to utilize the resources wisely while providing clock frequencies as high as $100 \mathrm{MHz}$.

Xilinx Microblaze [15] is one of widely used 32 bit softcore processors optimized for Xilinx FPGAs in the SoPC based embedded systems domain. Figure 2.8 shows the microarchitecture of Microblaze processor comes with the Xilinx tool chain. The processor is based on RISC load-store architecture and uses five or three stage pipeline depth depending on the area optimization settings. When targeting a Vertex II Pro FPGA, the Microblaze core can be clocked up to a maximum of $100 \mathrm{MHz}$. The processor can be configured to have a barrel shifter, hardware division and multiplication unit, an FPU, a memory management unit and a configurable directed mapped separate instruction and data caches. The gray colored blocks can be configured in and out depending on the requirements. The GNU compiler tools are automatically configured to the reconfigured architecture. For example, if the hardware multiplier is configured in,

the compiler replaces the appropriate high level multiplication codes with hardware multiply instructions. 
External interfaces of the processor are highly configurable within the EDK, and the processor supports several external bus interfaces including standard shared buses and point to point links. The Microblaze comes with separate data side and instruction side OPB or PLB interfaces with configurability. It also supports Local Memory Bus [32] which can be used to connect on chip Block RAM controller [33] to connect to a Block RAM Block [34]. This offers a fast and deterministic way of accessing a small amount of on-chip memory. The simplicity of the LMB protocol makes it possible to access the block RAM with single cycle latency. LMB is a single master bus with no need for an arbitrator. The Xilinx Cache Link (XCL) helps to offload the main OPB or PLB bus from memory accesses through the direct connection with the memory controllers. Fast Simplex Link [35] is a point to point FIFO based configurable interface with special instructions for the communication. This link is also used to attach custom peripherals.

\subsection{Real-time Operating Systems for SoPC Based Systems}

Almost all of SoPC based embedded systems use operating systems to coordinate the system behavior. Operating systems [36] provide the abstraction layer between the hardware and application software. The OS kernel provides a set of services that simplifies the application software design by hiding detailed underlying hardware architecture. Multiple task handling is one of core services provided by any OS kernel. This provides the illusion of concurrency by interleaving the execution of active tasks and sharing a single execution environment (the CPU and other resource) in a time multiplexed manner. The OS kernel also provides mechanisms for task synchronization, time management and message passing, and the higher layers of OS services such as GUI, networking, etc. are built on top of core OS services. 


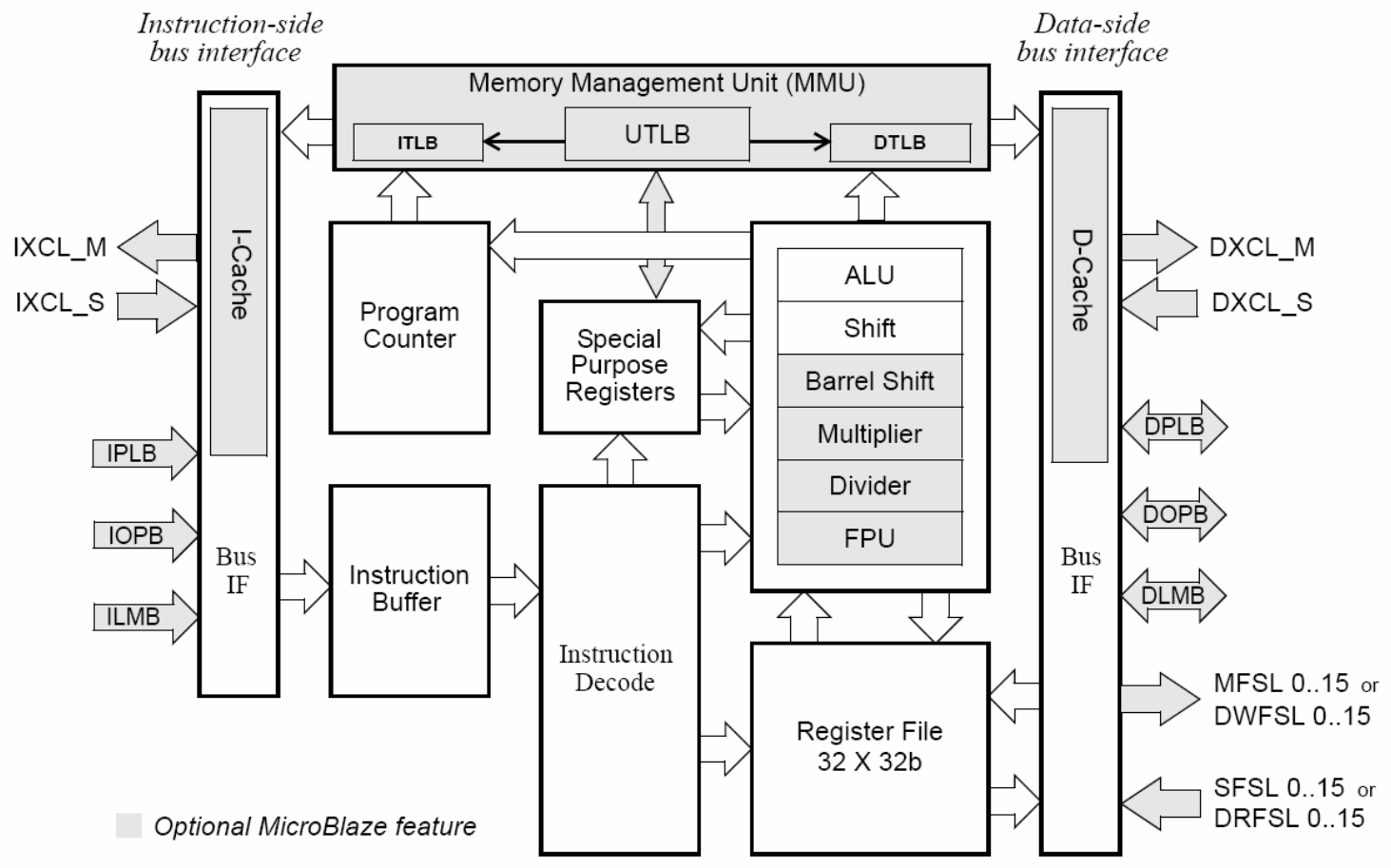

Figure 2.8: 32-Bit Microblaze Soft Processor [15]

Operating systems are usually categorized as either general purpose or real-time. The key differences between these two categorizations are the requirement of meeting the given deadlines of tasks and the determinism of the behavior of the system. Realtime systems are further categorized into soft real-time systems and hard real-time systems. Hard real-time systems have strict deadlines and although a missed deadline may even be catastrophic, this requirement is relaxed for the soft real-time systems. The determinism is one of main design goals of any real-time operating system. Simply put, given systems call needs to be serviced by the kernel within given time bounds. The general purpose OSs do not stress on such requirements resulting in very nondeterministic behavior, which is not an issue for the targeted application domains. For the guaranteed meeting of deadlines and increased determinism, the real-time operating systems are equipped with specialized scheduling algorithms [37]. The scheduling algorithm needs to be selected depending on the application context of the embedded system. For example, for the uniprocessor based hard real-time systems, the Earliest Deadline First (EDF) algorithm is capable of finding the optimal feasible schedule [38]. 
Xilkernal [39] is an open source small footprint robust software RTOS that comes with the Xilinx EDK tool chain. Integration with EDK helps to create SoPC systems running Xilkernal with little effort. Xilkernal provides a POSIX like interface with the ability to handle semaphores, interrupts, mutexes and message queues. Modularity of the kernel allows configurability of these modules. As a result, the kernel can be tailored for the specific needs of the given application. Xilkernal supports both priority-based preemptive scheduling and round robin scheduling. The policy is global and needs to be initialized at configuration time of the kernel.

\subsection{Multithreaded Processors}

Almost all modern processors use the concept of pipelining [40] in order to maximize the throughput of the processor. The pipelined execution divides each instruction into subparts. This leads to the overlapped execution of consecutive instructions. If two data dependant instructions are being executed in the pipeline, the current instruction at the execution stage cannot obtain the latest value from the register file since the earlier instruction is still at the later stages of the pipeline where the write back of results occurs. This leads to a problem called data hazards, and this particular type is known as Read After Write (RAW) hazards.

Figure 2.9 illustrates the RAW hazards progress in a pipeline. For example, the "AND" instruction requires the value in register R1 during the $3^{\text {rd }}$ cycle, but the value is written to the register file only after $5^{\text {th }}$ stage by the "ADD" instruction. The "OR" and "SUB" instructions also use the value in R1 before the register file is updated with its latest value. 


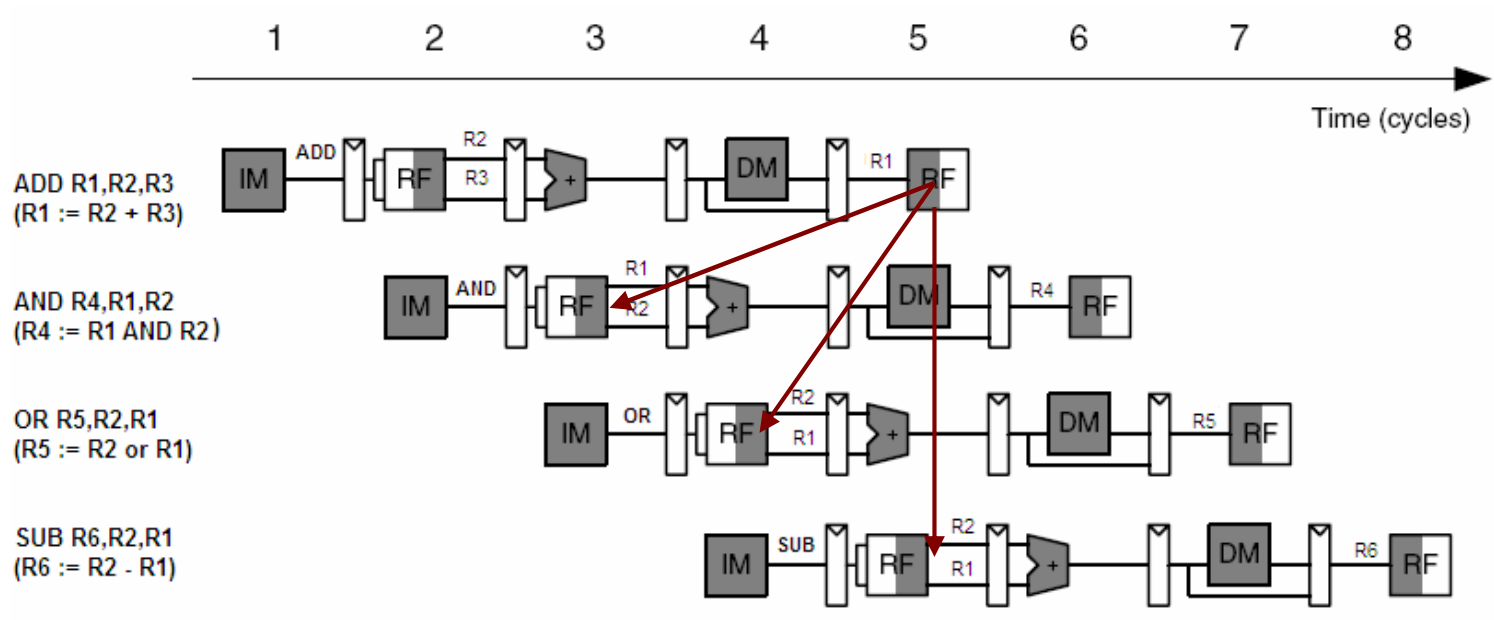

Figure 2.9: Pipeline Hazards Due to Data Dependencies

Most of the RAW hazards can be eliminated using a technique called data forwarding. Figure 2.10 illustrates the possibility of RAW hazard elimination using the data forwarding. Since the results of "ADD" operation are available at the beginning of the $4^{\text {th }}$ cycle, the results can be forwarded to the input of the ALU during the same cycle. Therefore, the "AND" instruction can use the correct value of R1 directly from the result generated by "ADD" instruction. The hazard situation of the "OR" instruction can also be eliminated by forwarding.

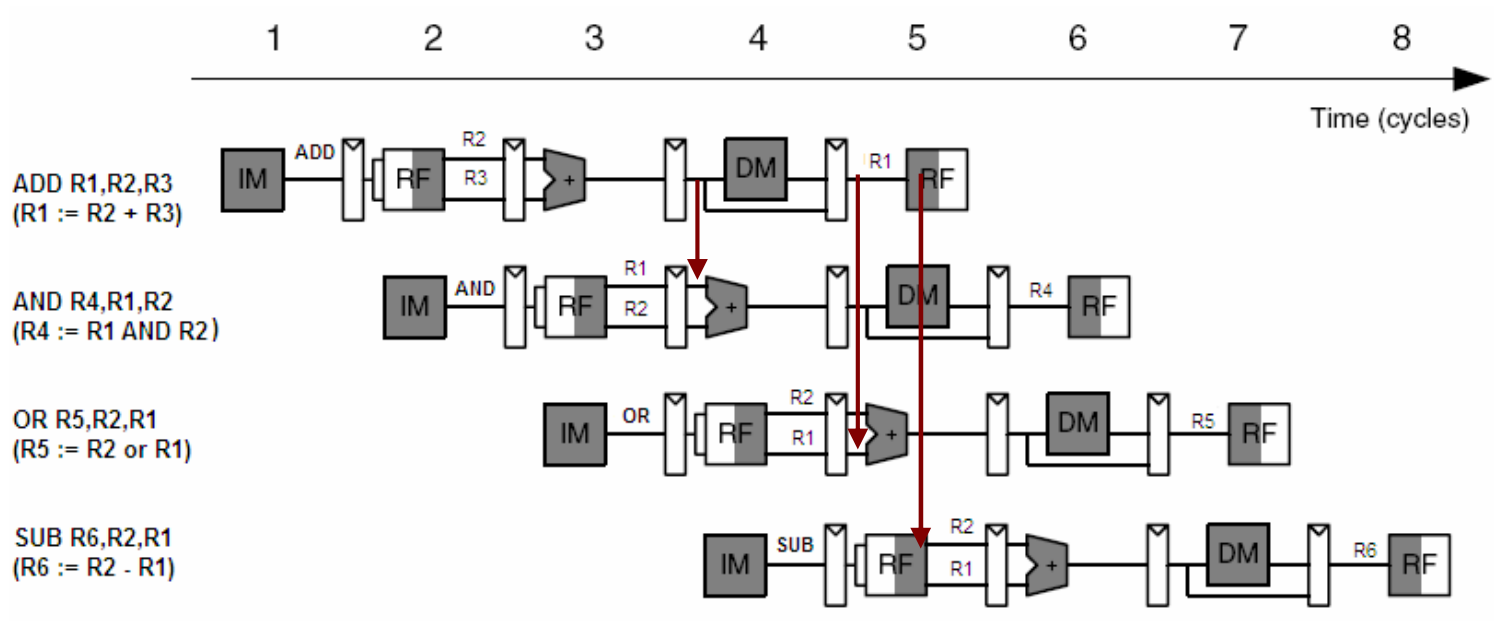

Figure 2.10: RAW Hazard Resolution with Data Forwarding 
Data forwarding is not always capable of resolving RAW hazards. For example, if the "ADD" instruction was changed into a "LOAD" instruction to load R1 from the data memory, the up-to-date value of R1 would be available only after the $4^{\text {th }}$ cycle. However, the "AND" instruction needs the value of R1 at the beginning of the $4^{\text {th }}$ cycle. Pipeline stall is required to overcome this hazard. This is done by freezing the first and second stages during the $4^{\text {th }}$ cycle and inserting a bubble into the $3^{\text {rd }}$ stage.

Since the instructions are overlapped, the execution of branch instructions causes another kind of hazards called control hazards. At the time a branch is resolved, depending on the design of the pipeline, several instructions may be in progress. If the branch is taken, all those instructions need to be flushed and pipeline has to be restarted from the branch target address. Occasionally, branch delay slots are used to insert independent instructions where those instructions always get executed irrespective of the outcome of the branch instruction. The technique of branch prediction is used to overcome the penalty due to control hazards by predicting the outcome of the branch and to prefetch the target address in advance.

Multithreading [10] is another technique used to remove these penalties by interleaving instructions from different threads without adding much complexity to the processor pipeline. This technique does not improve the Cycles Per Instruction (CPI) of the processor, but exploits the parallelism among threads. Multithreading comes in cycle interleaved and block interleaved forms. Cycle interleaved multithreading [10] eliminates both branch penalty and pipeline hazards by interleaving instructions from different threads in every cycle. Multithreading is an attractive option to reduce the pipeline hazards, but it does not increase the performance of a single threaded execution.

Block multithreading [10] is the coarse grained version of the cycle interleaved multithreading where each thread is scheduled to run several cycles before switching to another thread. Threads are switched on events like cache misses and taken branches, so the processor can exploit the parallelism among the threads and can do useful work while the cache line is being fetched. This technique can be used to hide the memory latency in real-time systems [41]. Unlike the cycle interleaved case, all the forwarding logic and hazard detection need to be implemented, since each thread needs to have the possibility of running exclusively in the pipeline without being interrupted. 
Multithreading is implemented by providing architectural extensions to the pipeline to handle multiple threads at the hardware level. Conversion from a single threaded processor to a multithreaded processor is fairly simple. The instruction fetch stage should contain multiple program counters and a mechanism for switching the threads. The decode stage needs to have a duplicated register file, where a separate register is set for each thread. This duplication demands a larger register file. However, the requirement of duplicated register file can be relaxed at the cost of increased context switching time by using external tightly coupled context memory. Apart from the duplicated register file, the multithreaded processors pipeline needs to carry thread IDs to distinguish how the instructions belong to different threads. Since multithreaded processor can have instructions from different threads in its pipeline, the thread IDs needs to be propagated down the pipeline in order to associate each instruction with the relevant thread.

\subsection{Hardware Support for Operating Systems}

Several independent research groups have focused on the hardware implementation of the RTOS to take advantage of parallelism inherent to the hardware. The first OS implementations in hardware date back to 1995. This section investigates the previous research which mostly address on the separate, chip based migration of software RTOSs into hardware.

The Real Time Unit (RTU) [1] implements complete RTOS functionality on an Application Specific Integrated Circuit (ASIC). This chip works in parallel with the CPU and sits on the processor bus and communicates through a set of memory mapped registers. The main interrupt line of the processor is driven by this chip whenever a task switch is required. Upon interruption, the CPU reads the NEXT TASK ID register of the RTU and performs a context switch to run the newly selected process. The context switching is done in software by saving and loading the registers in the task control blocks. In this manner the CPU is offloaded from the kernel timer tick handling and the process scheduling. Therefore, the processor cycles consumed on these can be effectively used. This RTU uses static priority preemptive scheduling and supports up to 128 tasks with 8 priority levels. This RTU handles interrupts, periodic start of tasks, 
delay tasks and activation and deactivation of tasks by itself. FASTCHAT [9] project mates the above mentioned RTU with special CPU where it can perform the context switching in one cycle. This is a very simple single cycle non-pipelined CPU with two copies of the register file and other system and status registers. One set is used by the CPU while the other set is used by the RTU. Once the RTU comes to the decision of switching the task, it propagates its register file with the saved values in the Task Control Block (TCB) corresponding to the new task to be run and interrupts the CPU. Once interrupted, the CPU can immediately start executing the new task by switching to the register set field by RTU. RTU94 [42] further extends the concept into the multiprocessor domain by adding support to schedule processes in a multiprocessor system. The real-time unit still sits on the shared bus, and each CPU has access to a dedicated task register resides in the RTU. Unlike a single processor system, the policy selection for the shared bus arbitration has a direct impact on the predictability of the RTU calls. The only option is to use the round robin bus arbitration in order to be able to calculate a maximum access time for the RTU. The nonpredictability of the RTU calls increases further for the systems which employ bus locking mechanisms to provide atomic transactions. When it comes to advanced bus based systems, where the dynamic priority based arbitration is the natural selection, it is not possible to find a maximum bound in order to characterize the timing model of the RTU, resulting in nondeterminism. The RTU has been evolved further into a commercial product in the form of an intellectual property named Sierra [43] by RealFast AB. This IP comes as an IP addition [44] for the EDK.

The silicon TRON project [2] implements an RTOS in hardware by offloading primitive OS functions from the CPU. Even though this one is conceptually equivalent with the RTU, it has a thin software layer to meet the specifications of the ITRON project.

The Spring Scheduling Co-Processor (SSCoP) [3] is a noticeable deviation of hardware based realization of real-time kernels. This coprocessor accelerates the scheduling algorithm used by the Spring distributed real-time computing system [45]. In the context of single processor systems with preemptable tasks, the EDF and Least Laxity First (LLF) algorithms are optimal dynamic scheduling algorithms. That is, if 
those algorithms can't find a feasible schedule no other algorithms can find a schedule. But when it comes to the multiprocessor systems no optimal algorithms exist. In order to find a full feasible scheduling in a multiprocessor system a heuristics based approach needs to be used. SSCoP realizes a dynamic planning based scheduling algorithm with a heuristic scheduling approach. It also supports static scheduling and different policies including EDF. The schedule is made online as the tasks arrive, and if feasible scheduling is found the newly arrived task is accepted. The SSCoP is implemented in VLSI and has an asynchronous PROM interface in order to simplify the system interface. Communication is done through polling the memory interface making the interfacing inefficient and nondeterministic.

Similar to the SSCoP, Real Time Manager (RTM) [4] provides hardware support for real-time operating systems which lies in the middle of pure hardware based solutions and the pure software based solutions. The RTM provides a hardware based solution for task management, time management and event management. RTM provides a generic interface to the software and it can be easily integrated with existing RTOSs. RTM is also a memory mapped peripheral and can be accessed to get the secluded task to be run.

The configurable hardware scheduler [8] is also a memory mapped IP based solution proposed to offload the kernel overhead from the CPU. The main exception is, the IP is targeted for the configurable space, and it can be configured to use the following three scheduling algorithms: priority, earliest deadline first and rate monotonic algorithms. The IP core comes with a configuration utility that allows the number of tasks, number of external interrupts and timer resolution to be configured. The scheduler is implemented using two priority queues. The base architecture remains the same for each scheduling policy configured, but the sorting of the elements is done according to the queue element fields depending on the scheduling policy being used.

Some research groups [5,6] have focused on migrating only the scheduler component of the kernel into hardware. Their main focus is to implement efficient and complex scheduling algorithms in hardware, which otherwise is very inefficient with a pure software based approach. Saez, et al. [6], proposes a hardware implementation to address the issue of impractical software implementation of the slack stealing algorithm 
which is efficient for jointly scheduling both hard deadline periodic tasks and soft aperiodic tasks.

Partitioning between implementation in hardware and implementation in software of the real-time kernels has also been investigated by several research groups in order to analyze the possible speedup while using minimal hardware resources. The SoC/RTOS codesign framework [46] allows the designer to selectively migrate Atalanta [47] kernel components into hardware. This fine grained partitioning helps to achieve significant speedup with minimum hardware resources. A hardware/software kernel for SoC design [48] proposes an efficient way to partition the EDF scheduler into software and hardware implementations. This research further evaluates various options for software/hardware partitioning.

Previous research work proves that the hardware migration of partial or full kernel improves the overall performance of the RTOS. Apart from the commercialization efforts such as RealFast [43], which is fairly successful, current commercial RTOS are fully based on software implementations. Several factors contribute to this unattractiveness of the hardware based solution, since most research work is focused on silicon implementations. Historically all these implementations are based on dedicated silicon, residing external to the CPU and number of tasks supported by the hardware RTOS is fixed.

\subsection{Processors with OS Level Support}

The research work described earlier focused on the hardware migration of RTOS to remove the OS overhead and mainly focused on scheduler migration. The context switching overhead also contributes to a significant amount of OS overhead. Every time the OS performs a context switch, the CPU's architectural registers need to be saved and the new states related to the context being restored have to be loaded. In order to remove this overhead, the special processor has to be used. This section investigates processors with OS level support, including efficient context switching and interrupt handling.

When it comes to the domain of general purpose processors, some of those processors have special support for the efficient context switching including IA 32 [49] based processors. The Task State Segments (TSS) stores all the state information related 
to each task, and the processor performs the restoration transparently and efficiently. Apart from these types of supports, most general purpose processors do not provide considerable OS level support for efficient context switching.

The Transputer [50] has the ability to handle multiple concurrent tasks. It is the first known processor implementation with OS level support in a very limited fashion. Transputer implements a round robin scheduler for task scheduling, timer queues and some special instructions to make the thread sleep for a specified time. It uses the technique of windowing registers to implement fast context switches. Software Defined Silicon [51] is a new multiprocessor architecture stemmed from the Transputer architecture.

Several research works have been done to exploit excellent context switching characteristics of multithreading processors in the domain of real time systems. The first method is to use the fast context switching characteristic to hide the memory access latency in real time systems [41]. The second method is to use fast context switching ability to implement responsive interrupt service threads, which are directly executed upon the event of an external interrupt. Komodo [11] is a 4-way multithreaded processor with a hardware supported event handling mechanism based on hardware interrupt service threads. This processor lies between cycle interleaved multithreading and block interleaved multithreading. The processor supports four hardware threads with duplicated register files which can be used as Interrupt Service Threads (IST). The instruction fetch stage of Komodo is feasible for implementing several scheduling schemes. When it comes to the handling of multiple hard real-time events simultaneously, the guaranteed percentage scheduling scheme outperforms the optimal EDF algorithm by guaranteeing not only that the deadline is met but also the sustained data rates by interleaving the active threads. The zero context switching overhead helps achieve this by implementing a very fine grained implementation of the guaranteed percentage scheduling algorithm. The Komodo project has been further extended to implement a Java based microcontroller [12-14] targeting real-time applications. Three hardware threads are used for real-time threads, and the remaining one is shared among non real-time threads. 
Jackknife [52] is an AVR compatible multithreaded processor supporting 8 hardware threads. This processor also implements the ISTs with hardware support. The instruction fetch stage implements a round robin scheduler to interleave the active threads and interrupts are serviced in next cycle. Jackknife supports a synchronization mechanism using an external memory mapped peripheral.

The PRESTOR-1 [53] implements a virtual number of hardware contexts using a special context swapping architecture. Based on the virtual context architecture of the PRESTOR, a RTOS kernel [54] based on ITRON is proposed. This processor uses cache memory with priority based partitioning and provides support for fast interrupt processing using the multiple context support.

Java language itself has support for multithreading. The JVM maps those software threads to the OS threads depending on the JVM implementation. Significant research has been done in the area of hardware implementation of JVM. Java Optimized Processor (JOP) [55] is a processor that executes JAVA byte code at the hardware level. Several commercialized architectures such as ARM, provides hardware level JVM implementations in order to accelerate java byte code execution. However, hardware implementation of Java virtual machine causes considerable overhead and is not suitable for hard real-time systems. 


\section{Chapter 3}

\section{Design and Implementation of the RTBlaze}

This thesis combines the earlier research work on both hardware realizations of RTOSs and multithreaded processors to design a new processor, RTBlaze, with tightly coupled hardware RTOS. RTBlaze is a multithreaded processor that integrates a static priority based preemptive scheduler, timer and semaphore handling queues to the pipeline.

The first part of this chapter describes the design of the base processor compatible with MicroBlaze. The second part of this chapter discusses the architectural modifications made to the base processor in order to augment the instruction set with RTOS level support. 


\subsection{Design of the Base Processor}

Due to the availability of the EDA tool chain for SoPC design and the GNU compiler tool chain from Xilinx, the base processor is designed to be an instruction set compatible with basic MicroBlaze. This compatibility enables seamless integration of the base processor with the Xilinx tool chain and reusability of library IP cores with the existing device drivers. To be compatible, the base processor needs to do the following.

- Implement the bus standards supported by MicroBlaze

- Be compatible MicroBlaze Instruction set

- Be cycle accurate with MicroBlaze

Since the base processor implementation is targeted for the demonstration of the hardware RTOS concept, a minimalist implementation of the base processor is carried out with following components.

- A 32 × 32 bit register file

- A special purpose registers

- An ALU

- An instruction side LMB memory interface

- A data side LMB memory interface

Thus, exception handling, PLB interface, Fast Simplex Links, and the configurable parts of the MicroBlaze are not implemented.

The processor features a 32 bit address bus with memory mapped IO. Even though the processor uses Harvard architecture with split instruction and data memory interfaces, the EDK overlaps both address space by mapping both interfaces to the same memory block through LMB to Block RAM controller IP as shown in Figure 3.1. Apart from the memory controllers, other custom peripherals can be connected to the LMB bus within the EDK. 


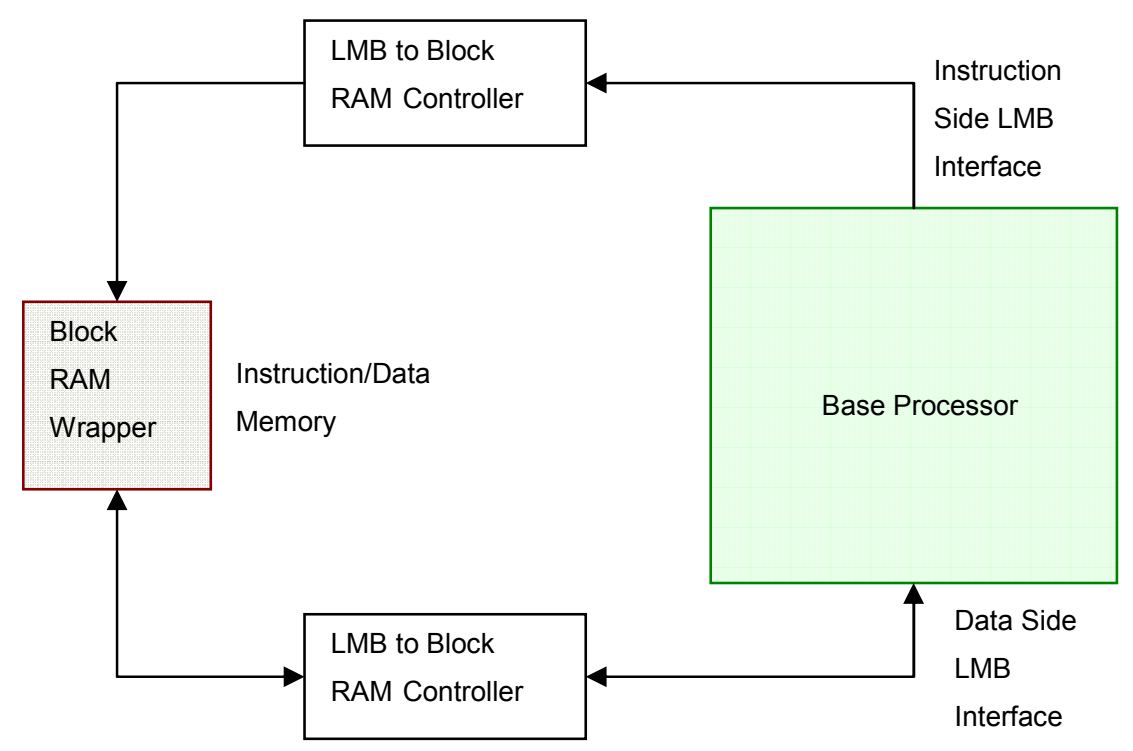

Figure 3.1: EDK Integration of the Base Processor

\begin{tabular}{|c|c|c|c|c|}
\hline Fetch & Decode & Execute & Mem. Access & Write back \\
\cline { 3 - 5 } & Fetch & Decode & Execute & Mem. Access \\
\cline { 3 - 5 } & Fetch & Decode & Execute \\
\cline { 3 - 4 } & & Fetch & Decode \\
\cline { 3 - 4 } & & & Fetch \\
& &
\end{tabular}

Figure 3.2: The Five Stage Pipeline

The base processor implements minimalist MicroBlaze instruction set using a five stage pipeline as shown in Figure 3.2. Figure 3.3 shows the simplified pipeline schematic of the base processor. The fetch stage retrieves the 32-bit fixed size, word aligned instruction from instruction side LMB. The Program Counter (PC) is also updated to point to the next instruction during this stage. The decoding stage performs the instruction decoding and control signal generation. The register file is also addressed with corresponding register address fields of the instruction. Execution stage performs the operation specified in the opcode of the instruction using the ALU. Memory stage performs data read and write operations with the support for different data widths through the data side LMB bus connected to on chip block RAM. The write back stage writes the result into the register file. The pipeline carries the data path signals as well as control signals to each stage. The hazard detection and control unit performs the freezing and flushing of the pipeline depending on the hazard situation in progress. Based on the 
proposed pipeline model, the compatibility with the Microblaze instruction set can be achieved by carefully adding specific support for each instruction. Decisions related to the internal architecture can be relaxed provided that the base processor can execute the same instructions with exactly the same results and latencies.

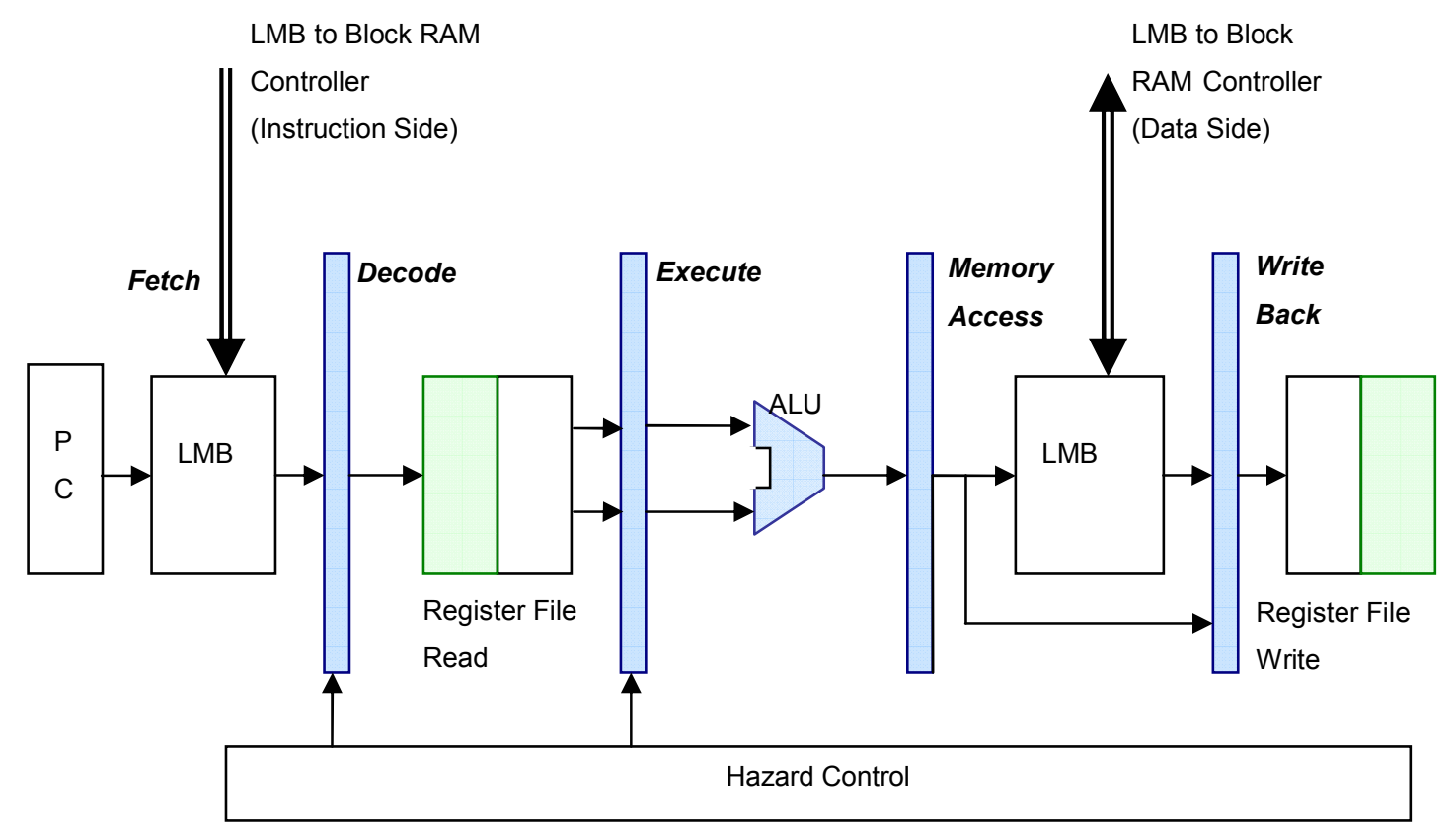

Figure 3.3: Block Diagram of the Proposed Base Processor

\begin{tabular}{|l|l|l|l|l|l|l|l|l|l|l|l|l|l|l|}
\hline Opcode & Destination Reg & Source Reg A & Source Reg B & 0 & 0 & 0 & 0 & 0 & 0 & 0 & 0 & 0 & 0 & 0 \\
\hline 0
\end{tabular}

\begin{tabular}{|l|l|l|lc|}
\hline Opcode & Destination Reg & Source Reg A & & Immediate Value \\
\hline 0 & 6 & 1 & 1 & 3 \\
& 6 & 1 & 6 & 1
\end{tabular}

Source Reg A - Ra

Source Reg B - Rb

Destination Reg - Rd

Figure 3.4: The Instruction Formats 
Table 3.1: A Partial List of Microblaze Instruction Set

\begin{tabular}{|c|c|c|}
\hline Group & Instruction & Semantics \\
\hline \multirow{4}{*}{$\begin{array}{c}\text { Arithmetic, Logical and } \\
\text { Shifting instructions }\end{array}$} & ADD Rd,Ra, Rb & $\mathrm{Rd}:=\mathrm{Rb}+\mathrm{Ra}$ \\
\hline & ADDI Rd,Ra,Imm & $\mathrm{Rd}:=\mathrm{s}(\mathrm{Imm})+\mathrm{Ra}$ \\
\hline & RSUB Rd,Ra, Rb & $\mathrm{Rd}:=\mathrm{Rb}+\mathrm{Ra}+1$ \\
\hline & OR Rd,Ra,Rb & $\mathrm{Rd}:=\mathrm{Ra}$ or $\mathrm{Rb}$ \\
\hline \multirow[t]{2}{*}{ Load/Store instructions } & LW Rd,Ra,Rb & $\begin{array}{l}\text { Addr }:=\mathrm{Ra}+\mathrm{Rb} \\
\mathrm{Rd}:=* \text { Addr }\end{array}$ \\
\hline & $\mathrm{SW} R d, \mathrm{Ra}, \mathrm{Rb}$ & $\begin{array}{l}\text { Addr }:=\mathrm{Ra}+\mathrm{Rb} \\
\text { *Addr }:=\mathrm{Rd}\end{array}$ \\
\hline \multirow{6}{*}{$\begin{array}{c}\text { Control transfer } \\
\text { instructions }\end{array}$} & BRLD Rd,Rb & $\begin{array}{l}\mathrm{PC}:=\mathrm{PC}+\mathrm{Rb} \\
\mathrm{Rd}:=\mathrm{PC}\end{array}$ \\
\hline & BRA Rb & $\mathrm{PC}:=\mathrm{Rb}$ \\
\hline & BEQ Ra,Rb & $\mathrm{PC}:=\mathrm{PC}+\mathrm{Rb}$ if $\mathrm{Ra}=0$ \\
\hline & RTSD Ra,Imm & $\mathrm{PC}:=\mathrm{Ra}+\mathrm{s}(\mathrm{Imm})$ \\
\hline & BRAI Imm & $\mathrm{PC}:=\mathrm{s}(\mathrm{Imm})$ \\
\hline & BEQI Ra,Imm & $\mathrm{PC}:=\mathrm{PC}+\mathrm{s}(\mathrm{Imm})$ if $\mathrm{Ra}=0$ \\
\hline
\end{tabular}

The MicroBlaze instructions have two main formats as shown in Figure 3.4. All MicroBlaze instructions are defined as either Type A or Type B [15]. Type A instructions have up to two source register operands and one destination register operand. Type B instructions have one source register and a 16-bit immediate operand. Sign extension is used to extend the 16 bit immediate operand of the instruction into a 32 bit word by preserving the sign of the immediate value. Type $B$ instructions have a single destination register operand. Based on the execution patterns in the pipeline, the instruction set of Microblaze can be categorized into following groups.

1. Arithmetic, Logical and Shifting instructions

2. Load/Store instructions

3. Control transfer instructions

The Microblaze instruction set contains more than 100 instructions. A partial list of instructions in each group is shown in Table 3.1. In order to support the execution of those instructions, the data transfer paths in the pipeline need to be multiplexed. For example, one input of the ALU needs to be multiplexed between the sign extended 
immediate and register "Rb" in order to support both "ADD" and "ADDI" instructions. By analyzing the execution and data transfer patterns of each instruction, the data path of the base processor pipeline is synthesized as shown in Figure 3.5. For the second group, the data path is formed by the multiplexers " $\mathrm{A}$ ", "B", "D" and "E" while the first group bypasses the data memory through the multiplexer " $\mathrm{D}$ ". The third group uses the "A", "B" and " $\mathrm{C}$ " multiplexers to deliver the branch target address to the PC.

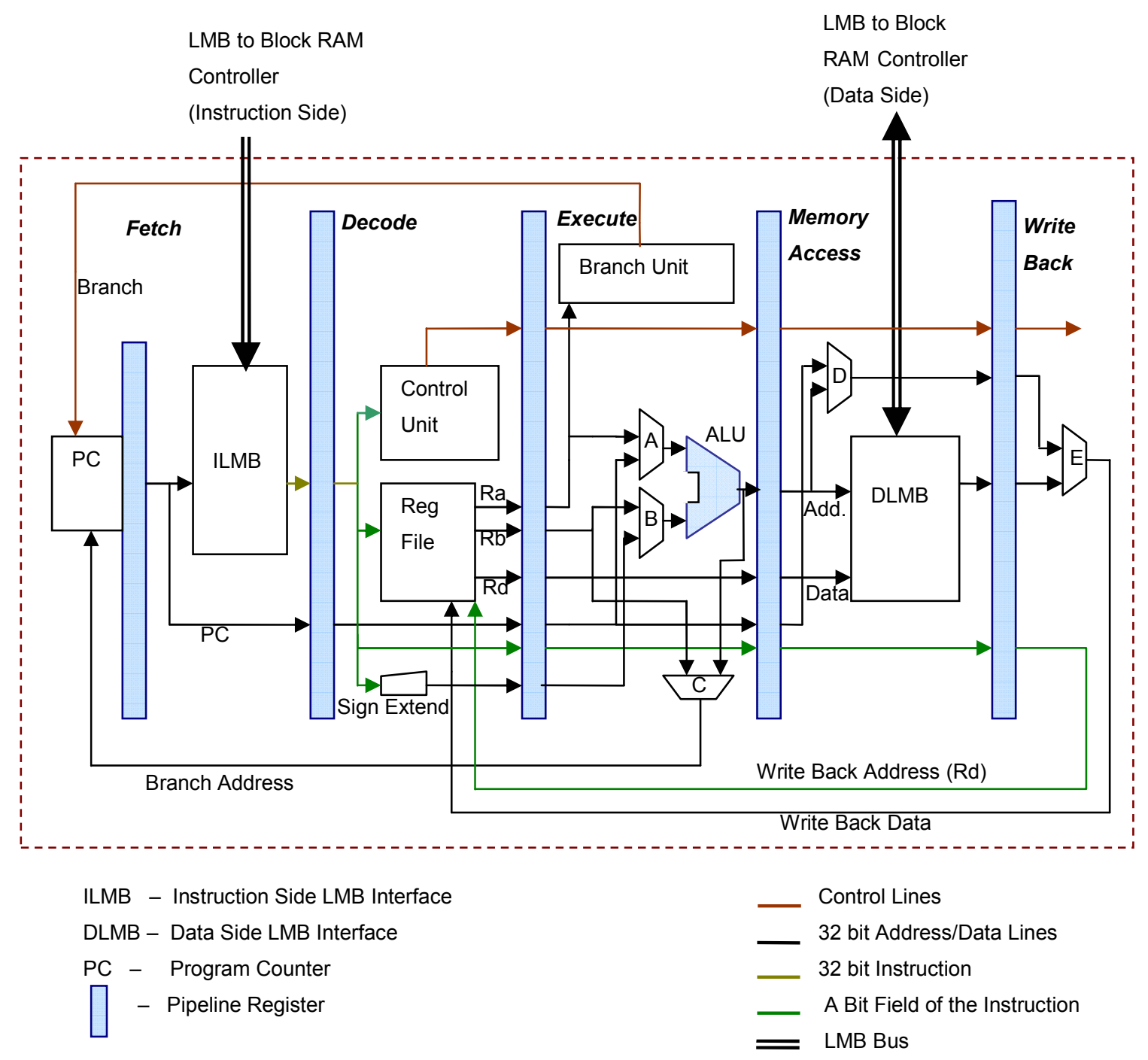

Figure 3.5: Simplified Five Stage Pipeline of the Base Processor

The first group of instructions, such as "ADD $\mathrm{Rd}, \mathrm{Ra}, \mathrm{Rb}$ ", have almost identical patterns of execution in the pipeline. The decoding stage uses the register address fields 
of the instruction to address the register file. The register values are forwarded to the execution stage in next cycle. The sign-extended value of the immediate field of the instruction is also forwarded to the execution stage. During the execution stage, the ALU is provided with relevant input values, and the operation is selected using the control signals present at the execution stage. In the memory access stage, the result is directly forwarded to the writeback stage, since this type of instruction does not perform any data memory access. The writeback stage accesses the register file to write the value to the destination register.

The load/store instructions access data memory using register indirect addressing. These instructions have three arguments, two source register fields and one source/destination register field. Both source operands are used to calculate the address of the memory location being accessed using the ALU. For the store instructions, the value in the destination register is written to memory during the memory access stage. The destination register is used to store the value read from the memory for the load instructions.

Control transfer instructions modify the program counter depending on a given condition. During the decoding stage, the register file is accessed to retrieve the values related to the condition evaluation. The condition is evaluated during the execution stage using special comparators. The ALU calculates the address of the branch target. If the condition is resolved to true, the program counter in the fetch stage is loaded with the newly calculated address.

The following sections provide a detailed architectural description of the base processor design. 


\subsubsection{First Stage - Instruction Fetch}

The microarchitecture of the fetch stage is shown in Figure 3.6. The fetch stage contains a presetable 32 bit Program Counter (PC). The value in the PC is forwarded to the LMB interface. The LMB interface performs the appropriate signal generation for the external read-only LMB [32] interface to get the current instruction from the memory. Once the instruction fetch is completed, the PC is incremented to point to the next instruction. The PC gets loaded for the branch instructions with the address calculated at the execution stage if a taken branch is in progress.
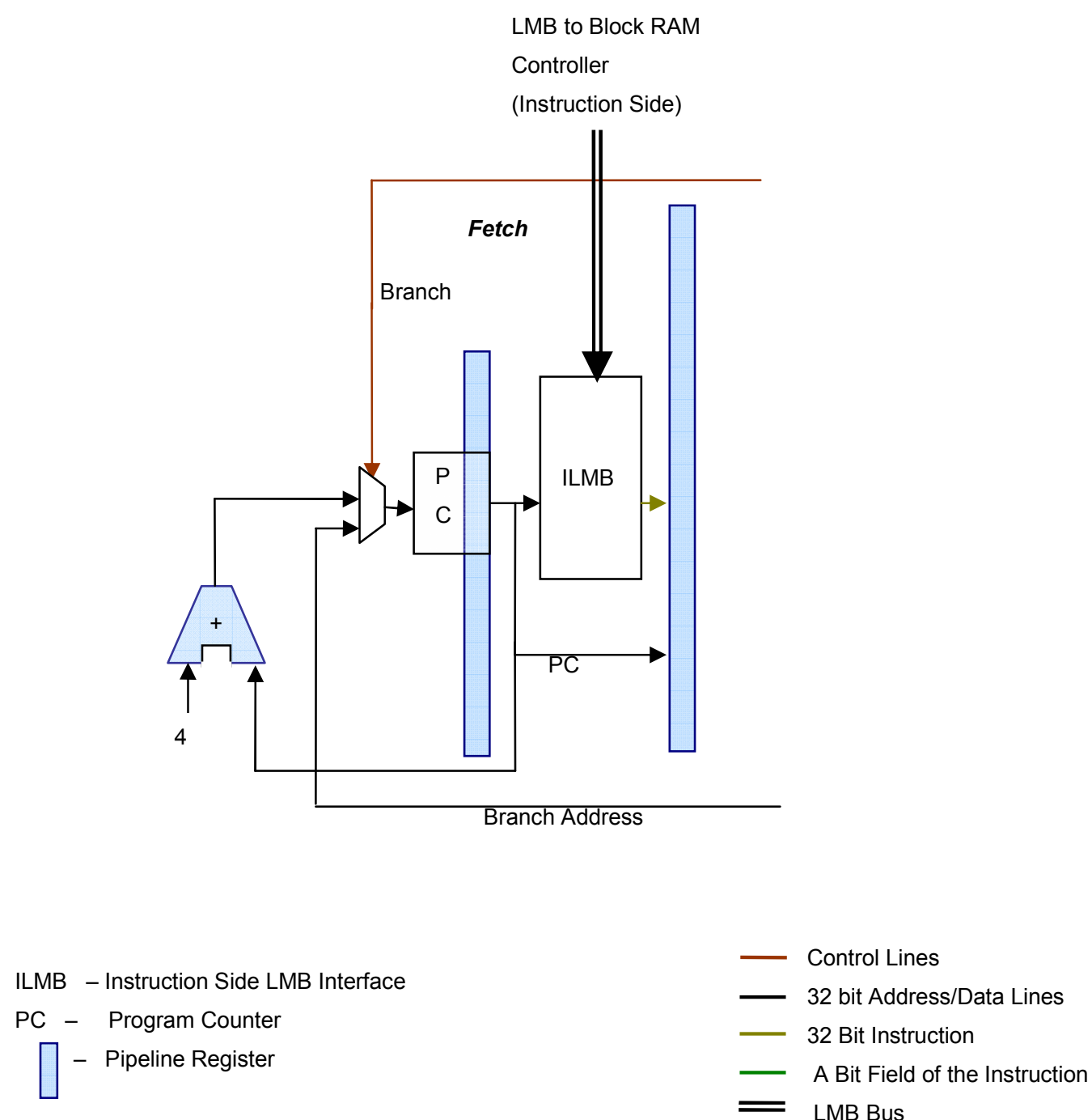

Figure 3.6: Instruction Fetch Stage 
Figure 3.7 shows the bus timing for a single read transaction of the LMB. Since the instruction side interface uses a read-only memory, only the read strobe is used. The byte enables are always fixed at logic high. LMB runs at full processor speed and is capable of pumping an instruction in every cycle.

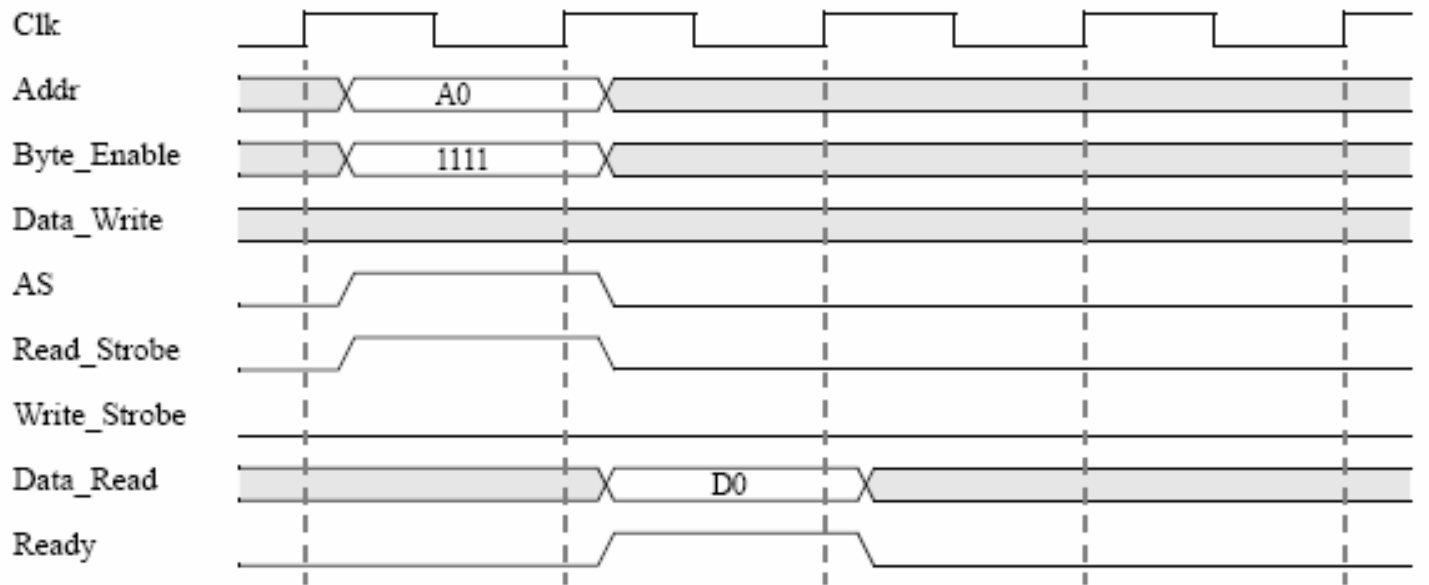

Figure 3.7: LMB Single Read Transaction [15]

\subsubsection{Second Stage - Instruction Decode}

This stage decodes the incoming instruction and generates appropriate control signals to coordinate the tasks performed in the execution, memory access and write back stages. Control signals are generated by a combinatorial logic circuit using the opcode filed of the instruction. Figure 3.8 shows the flow of control signals through each pipeline stage along with the instruction. This flow of control signal guarantees that each pipeline stage of the instruction processing receives the correct control signals. 


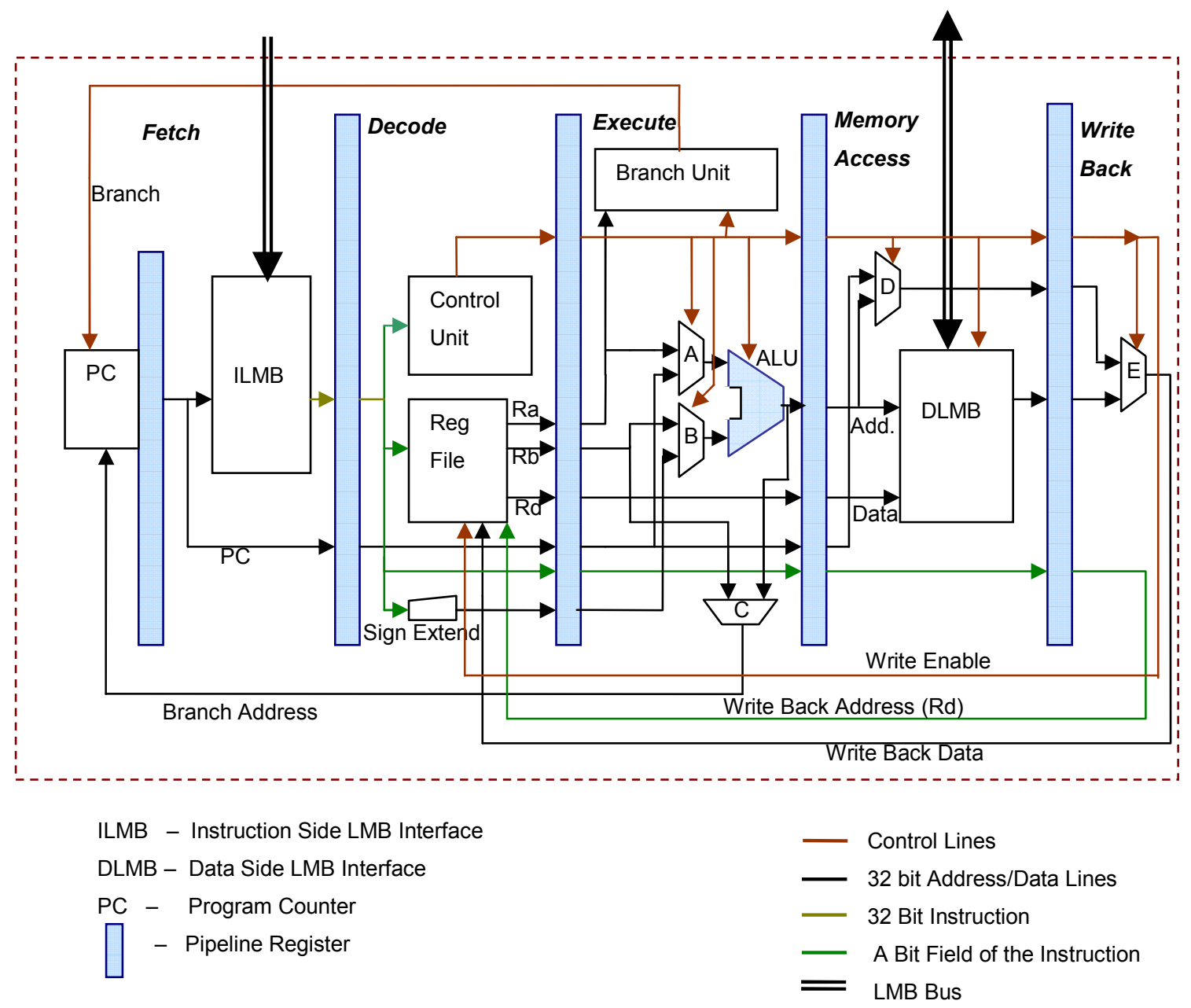

Figure 3.8: Control Signal Flow in the Pipeline

Table 3.2 shows the flow of control signals during the execution of two instructions. The highlighted signals are used as control signals for the corresponding stage, and the other signals are forwarded to the next pipeline stage. For example, when the ADD instruction enters the decoding stage of the pipeline, the control unit generates the signals to control the "A", "B", "D", "E" multiplexers, ALU and "write enable" of register file. During the next cycle, those control signals are propagated to the execution stage along with the register file outputs. The control signals relevant to the " $\mathrm{A}$ " and "B" multiplexer are used to route the "Ra" and " $\mathrm{Rb}$ " outputs of the register file to the ALU inputs. The ALU control signal is used to select the ALU function. The next cycle causes the result of the addition, control signals related to "D", "E" multiplexers and "write 
enable" to be propagated to the memory access stage. The " $\mathrm{D}$ " multiplexer directly forwards the result to the next pipeline stage. During the last cycle, the "E" multiplexer forwards the result of the addition to the write port of the register file, and the "write enable" signal causes the register file to be updated.

Table 3.2: Flow of Control Signals through Each Pipeline Stage

\begin{tabular}{|c|c|c|c|c|c|}
\hline & Fetch & Decode & Execute & $\begin{array}{l}\text { Mem. } \\
\text { Access }\end{array}$ & Writeback \\
\hline 1 & ADD Rd,Ra,Rb & & & & \\
\hline 2 & LW Rd,Ra,Rb & $\begin{array}{l}\text { ADD Rd,Ra,Rb } \\
M u x \_A=R a \\
M u x \_B=R b \\
A L U \_O P=a d d \\
M u x \_D=A L U \_o u t \\
\text { Mux_E= } \\
\text { mux_D_out } \\
\text { Regfile_WE=true }\end{array}$ & & & \\
\hline 3 & & $\begin{array}{l}\text { LW Rd,Ra,Rb } \\
M u x \_A=R a \\
M u x \_B=R b \\
A L U \_O P=a d d \\
\text { Mux_E=Mem_out } \\
\text { Regfile_WE=true } \\
\text { Mem_RE =true }\end{array}$ & $\begin{array}{l}\text { ADD Rd,Ra,Rb } \\
\boldsymbol{M u x} \boldsymbol{A}=\boldsymbol{R} \boldsymbol{a} \\
\boldsymbol{M u x} \boldsymbol{B}=\boldsymbol{R} \boldsymbol{b} \\
\boldsymbol{A} \boldsymbol{L} \boldsymbol{U} \_\boldsymbol{O P}=\boldsymbol{a d d} \\
\text { Mux_D=ALU_out } \\
\text { Mux_E= } \\
\text { mux_D_out } \\
\text { Regfile_WE =true }\end{array}$ & & \\
\hline 4 & & & 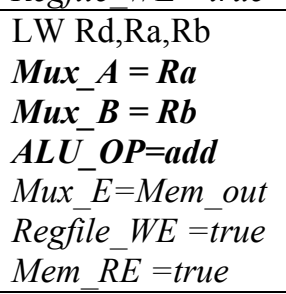 & $\begin{array}{l}\text { ADD Rd,Ra,Rb } \\
\text { Mux_D } \\
=\boldsymbol{A L} \boldsymbol{L} \boldsymbol{U}_{-} \text {out } \\
\text { Mux_E= } \\
\text { mux_D_out } \\
\text { Regfile_WE=true }\end{array}$ & \\
\hline 5 & & & & $\begin{array}{l}\text { LW Rd,Ra,Rb } \\
\text { Mux_E=Mem_out } \\
\text { Regfile_WE =true } \\
\text { Mem_RE }=\text { true }\end{array}$ & $\begin{array}{l}\mathrm{ADD} \mathrm{Rd}, \mathrm{Ra}, \mathrm{Rb} \\
\boldsymbol{M u x} \boldsymbol{E}=\boldsymbol{E} \boldsymbol{x} \_\boldsymbol{D} \_\boldsymbol{o u t} \\
\text { Regfile_WE}=\boldsymbol{t} \boldsymbol{t} \boldsymbol{e} \boldsymbol{e}\end{array}$ \\
\hline & & & & & $\begin{array}{l}\mathrm{LW} \mathrm{Rd}, \mathrm{Ra}, \mathrm{Rb} \\
\text { Mux_E=Mem_out } \\
\text { Regfile_WE=true }\end{array}$ \\
\hline
\end{tabular}

In addition to the instruction decoding, the register file is accessed during this stage. Three read ports provide the operand values to the next stage on the next negative clock edge. The corresponding bit fields of the instruction are used to address the threeport register file. Analysis of the Microblaze instruction set reveals that there are some 
instructions accessing three operands from the register file simultaneously. For example, the store instruction reads 3 registers from the register file in order to execute. To support three simultaneous reads during the decoding stage, the register file has to provide three read ports. Figure 3.9 illustrates the block diagram of the multiported register file. The register file with three read ports is realized using three copies of dual ported memory blocks and forwarding writes to all three memory blocks.

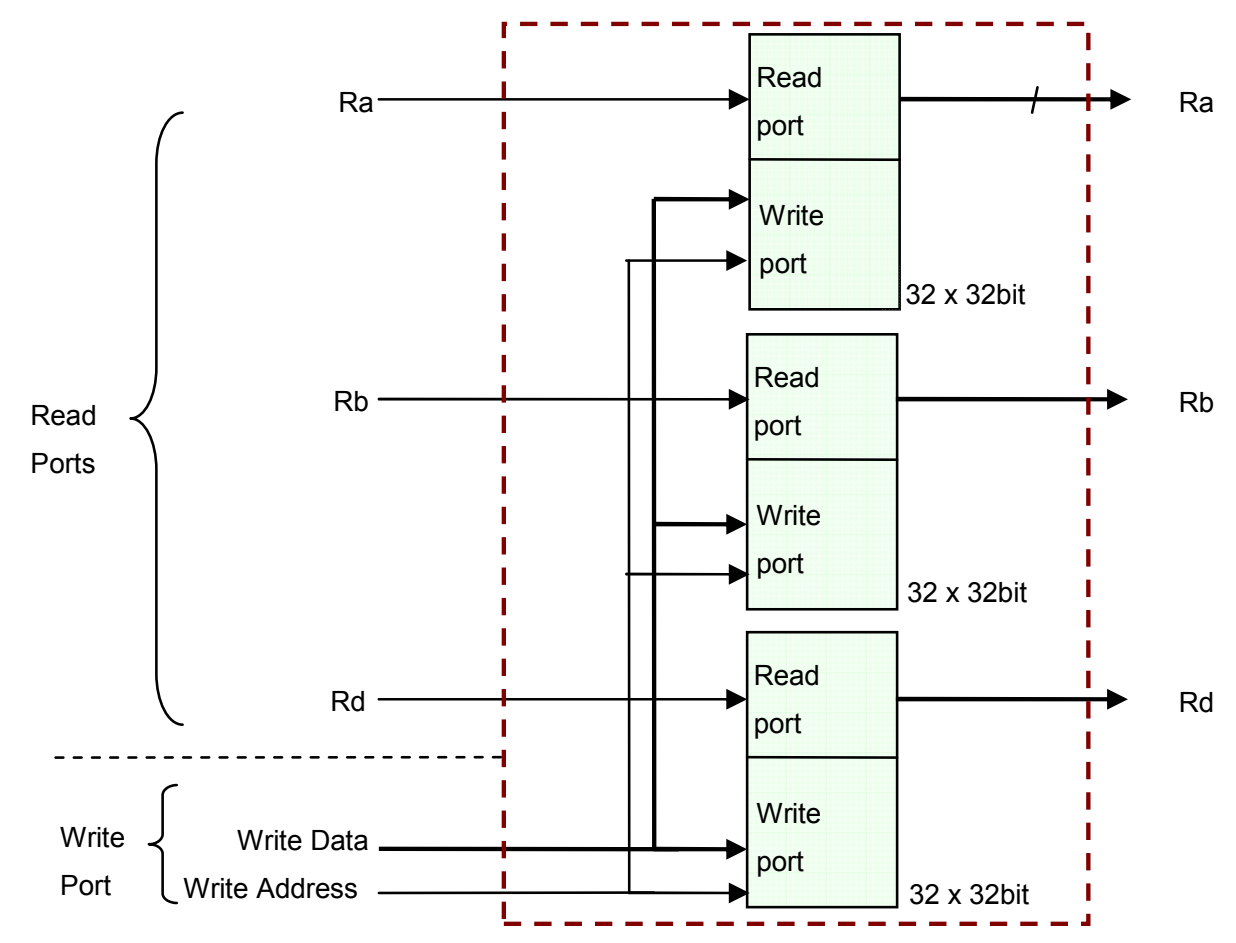

Figure 3.9: Register File with Three Read Ports and One Write Port

Signed extended immediate value is also generated in this stage by extending the 16 bit immediate field to a 32 bit field by preserving the sign of the original immediate filed. The result of the sign extension is directly forwarded along with the register file output to the execution stage, and multiplexer "B" switches between these two depending on the type of the instruction being executed. 


\subsubsection{Third Stage - Instruction Execute}

This stage performs the execution of the instruction using an arithmetic and logic unit. The logic unit supports four logical instructions, “OR”, “AND”, “XOR” and " $A N D$ NOT". The arithmetic unit supports signed/unsigned addition and subtraction. The operation of the ALU is summarized in the Table 3.3 .

Table 3.3: Operations Supported by the ALU

\begin{tabular}{|c|c|}
\hline ALU OP code & Operation \\
\hline 000 & $\mathrm{Ra}+\mathrm{Rb}$ \\
\hline 001 & $\mathrm{Ra}-\mathrm{Rb}$ \\
\hline 100 & $\mathrm{Ra}$ and $\mathrm{Rb}$ \\
\hline 101 & $\mathrm{Ra}$ or $\mathrm{Rb}$ \\
\hline 110 & $\mathrm{Ra}$ xor $\mathrm{Rb}$ \\
\hline 111 & $\mathrm{Ra}$ and not $\mathrm{Rb}$ \\
\hline
\end{tabular}

The status register is implemented in this stage and the ALU uses and updates the arithmetic carry bit of the status register based on the instruction type. This architecture does not use the statues bits for the condition evaluation of branch instructions. Instead, the instruction set uses dedicated comparators for the branch condition evaluation.

The execution stage also performs the branch resolution, branch and load/store memory address calculations. Apart from the unconditional branches, the instruction set includes conditional branches as listed in Table 3.4. Three dedicated comparators as shown in Figure 3.10 are used to resolve all six conditions listed in Table 3.4 by combining the three outputs. Once the branch condition is resolved, the branch target address is calculated by the ALU. Since the PC represents an address, the instruction memory addresses need to always be considered as unsigned values when performing branch address calculations. In order to accommodate arithmetic on memory addresses, unsigned values are also supported for one input of the arithmetic unit. Depending on the 
branch type, the calculated branch target value flows through multiplexer " $\mathrm{C}$ " to the fetch stage as shown in Figure 3.11.

Table 3.4: Conditional Control Transfer Instructions

\begin{tabular}{|c|c|}
\hline Instruction & Semantics \\
\hline BEQ $\mathrm{Ra}, \mathrm{Rb}$ & $\mathrm{PC}:=\mathrm{PC}+\mathrm{Rb}$ if $\mathrm{Ra}=0$ \\
\hline $\mathrm{BNE} \mathrm{Ra,Rb}$ & $\mathrm{PC}:=\mathrm{PC}+\mathrm{Rb}$ if $\mathrm{Ra} !=0$ \\
\hline $\mathrm{BLT} \mathrm{Ra}, \mathrm{Rb}$ & $\mathrm{PC}:=\mathrm{PC}+\mathrm{Rb}$ if $\mathrm{Ra}<0$ \\
\hline $\mathrm{BLE} \mathrm{Ra,Rb}$ & $\mathrm{PC}:=\mathrm{PC}+\mathrm{Rb}$ if $\mathrm{Ra}<=0$ \\
\hline BGT $\mathrm{Ra}, \mathrm{Rb}$ & $\mathrm{PC}:=\mathrm{PC}+\mathrm{Rb}$ if $\mathrm{Ra}>0$ \\
\hline BGE $\mathrm{Ra}, \mathrm{Rb}$ & $\mathrm{PC}:=\mathrm{PC}+\mathrm{Rb}$ if $\mathrm{Ra}>=0$ \\
\hline
\end{tabular}

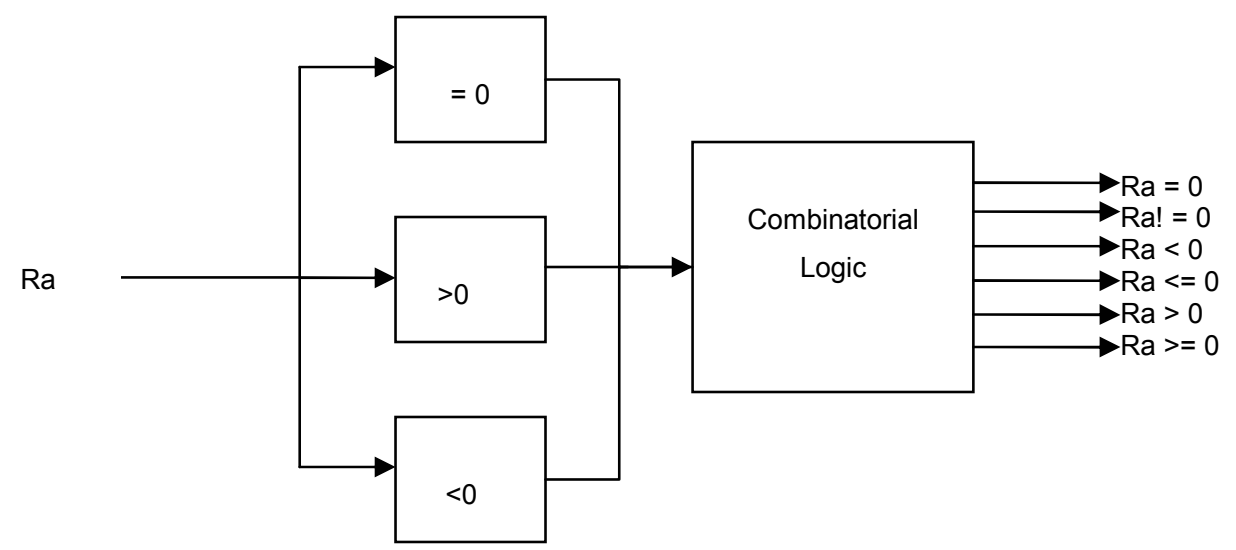

Figure 3.10: Branch Resolution Logic 


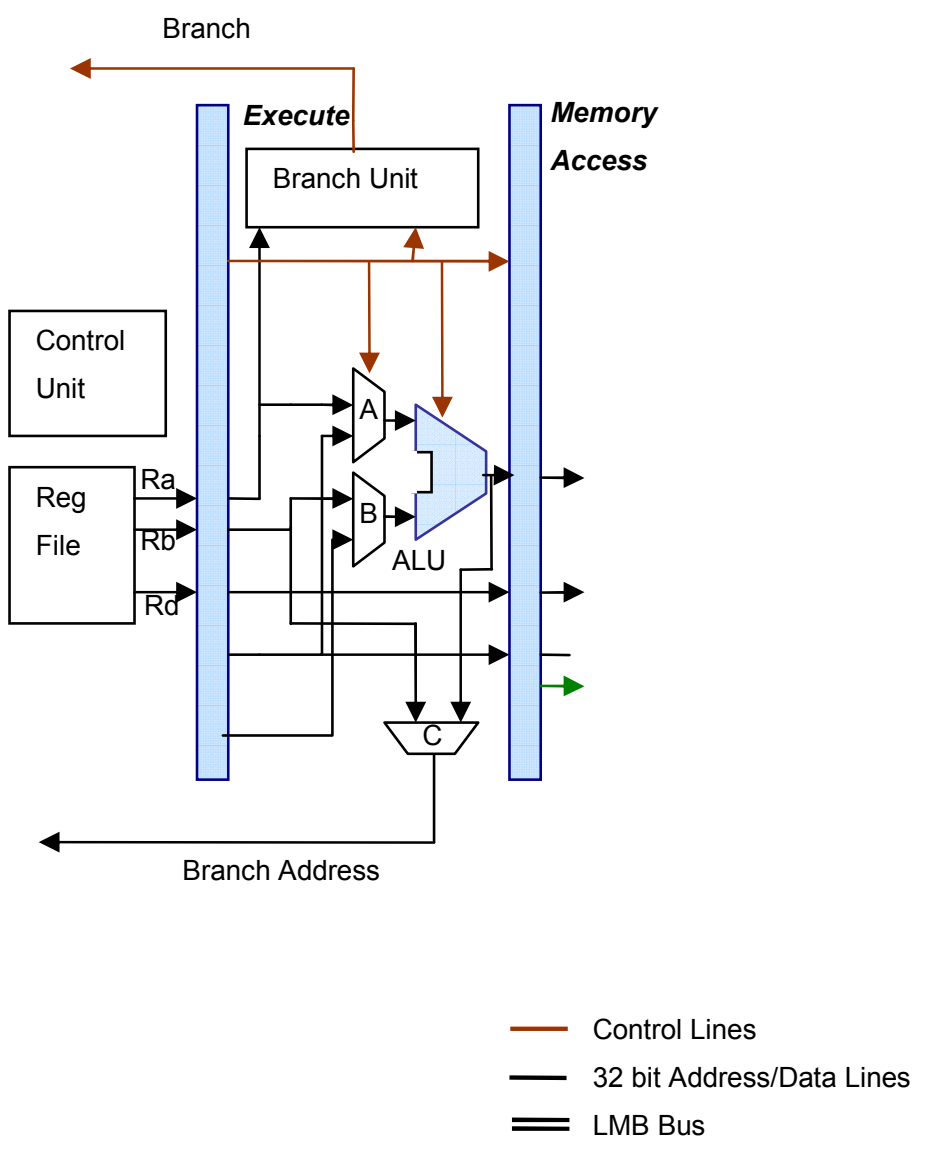

Figure 3.11: Execution Stage

\subsubsection{Fourth Stage - Data Memory Access}

This stage performs data memory access for the "Load" and "Store" instructions using the data side LMB interface. The memory address calculated during the execution stage is directly used to address the data memory. Microblaze has separate instructions for byte, half word, and word reads/writes as listed in Table 3.5. 
Table 3.5: Load Store Instructions

\begin{tabular}{|c|c|c|}
\hline Instruction & Semantics & Description \\
\hline LBU Rd,Ra, Rb & $\begin{array}{l}\text { Addr }:=\mathrm{Ra}+\mathrm{Rb} \\
\operatorname{Rd}[0: 23]:=0 \\
\operatorname{Rd}[24: 31]:=* \text { Addr[0:7] }\end{array}$ & Load byte unsigned \\
\hline LHU Rd,Ra, Rb & $\begin{array}{l}\text { Addr }:=\mathrm{Ra}+\mathrm{Rb} \\
\operatorname{Rd}[0: 15]:=0 \\
\operatorname{Rd}[16: 31]:=* \operatorname{Addr}[0: 15]\end{array}$ & $\begin{array}{l}\text { Load half word } \\
\text { unsigned }\end{array}$ \\
\hline LW Rd,Ra,Rb & $\begin{array}{l}\text { Addr }:=\mathrm{Ra}+\mathrm{Rb} \\
\mathrm{Rd}:=* \text { Addr }\end{array}$ & Load word \\
\hline $\mathrm{SB} \mathrm{Rd}, \mathrm{Ra}, \mathrm{Rb}$ & $\begin{array}{l}\text { Addr }:=\mathrm{Ra}+\mathrm{Rb} \\
* \text { Addr[0:8] }:=\mathrm{Rd}[24: 31]\end{array}$ & Store byte unsigned \\
\hline SH Rd,Ra,Rb & $\begin{array}{l}\text { Addr }:=\mathrm{Ra}+\mathrm{Rb} \\
* \operatorname{Addr}[0: 16]:=\mathrm{Rd}[16: 31]\end{array}$ & $\begin{array}{l}\text { Store half word } \\
\text { unsigned }\end{array}$ \\
\hline $\mathrm{SW} \mathrm{Rd}, \mathrm{Ra}, \mathrm{Rb}$ & $\begin{array}{l}\text { Addr }:=\mathrm{Ra}+\mathrm{Rb} \\
* \text { Addr }:=\mathrm{Rd}\end{array}$ & Store word \\
\hline
\end{tabular}

This stage also performs the data steering in order to perform byte and half word read and writes. When it comes to the byte reads/writes and half word reads/writes, the proper data byte needs to be steered to the proper byte location of the destination. Table 3.6 summarizes the read data steering mechanism, and Table 3.7 summarizes the data write steering mechanism. Figure 3.12 shows the architecture of the read data steering. The data steering is implemented using an array of multiplexers to route the correct byte to the correct location. The multiplexers are controlled depending on the data size of the operation and the address alignment (last 2 bits of the address). The write steering is implemented in a similar fashion.

Table 3.6: Read Data Steering [15]

\begin{tabular}{|c|c|c|c|c|c|c|}
\hline & & & \multicolumn{3}{|c|}{ Register rD Data } \\
\hline $\begin{array}{c}\text { Address } \\
\text { [30:31] }\end{array}$ & $\begin{array}{c}\text { Byte_Enable } \\
{[\mathbf{0}: 3]}\end{array}$ & $\begin{array}{c}\text { Transfer } \\
\text { Size }\end{array}$ & rD[0:7] & rD[8:15] & rD[16:23] & rD[24:31] \\
\hline 11 & 0001 & byte & & & & Byte3 \\
\hline 10 & 0010 & byte & & & & Byte2 \\
\hline 01 & 0100 & byte & & & & Byte1 \\
\hline 00 & 1000 & byte & & & & Byte0 \\
\hline 10 & 0011 & halfword & & & Byte2 & Byte3 \\
\hline 00 & 1100 & halfword & & & Byte0 & Byte1 \\
\hline 00 & 1111 & word & Byte0 & Byte1 & Byte2 & Byte3 \\
\hline
\end{tabular}


Table 3.7: Write Data Steering [15]

\begin{tabular}{|c|c|c|c|c|c|c|}
\hline & & & \multicolumn{4}{|c|}{ Write Data Bus Bytes } \\
\hline $\begin{array}{c}\text { Address } \\
{[30: 31]}\end{array}$ & $\begin{array}{c}\text { Byte_Enable } \\
{[0: 3]}\end{array}$ & $\begin{array}{c}\text { Transfer } \\
\text { Size }\end{array}$ & Byte0 & Byte1 & Byte2 & Byte3 \\
\hline 11 & 0001 & byte & & & & $\mathrm{rD}[24: 31]$ \\
\hline 10 & 0010 & byte & & & $\mathrm{rD}[24: 31]$ & \\
\hline 01 & 0100 & byte & & $\mathrm{rD}[24: 31]$ & & \\
\hline 00 & 1000 & byte & $\mathrm{rD}[24: 31]$ & & & \\
\hline 10 & 0011 & halfword & & & $\mathrm{rD}[16: 23]$ & $\mathrm{rD}[24: 31]$ \\
\hline 00 & 1100 & halfword & $\mathrm{rD}[16: 23]$ & $\mathrm{rD}[24: 31]$ & & \\
\hline 00 & 1111 & word & $\mathrm{rD}[0: 7]$ & $\mathrm{rD}[8: 15]$ & $\mathrm{rD}[16: 23]$ & $\mathrm{rD}[24: 31]$ \\
\hline
\end{tabular}

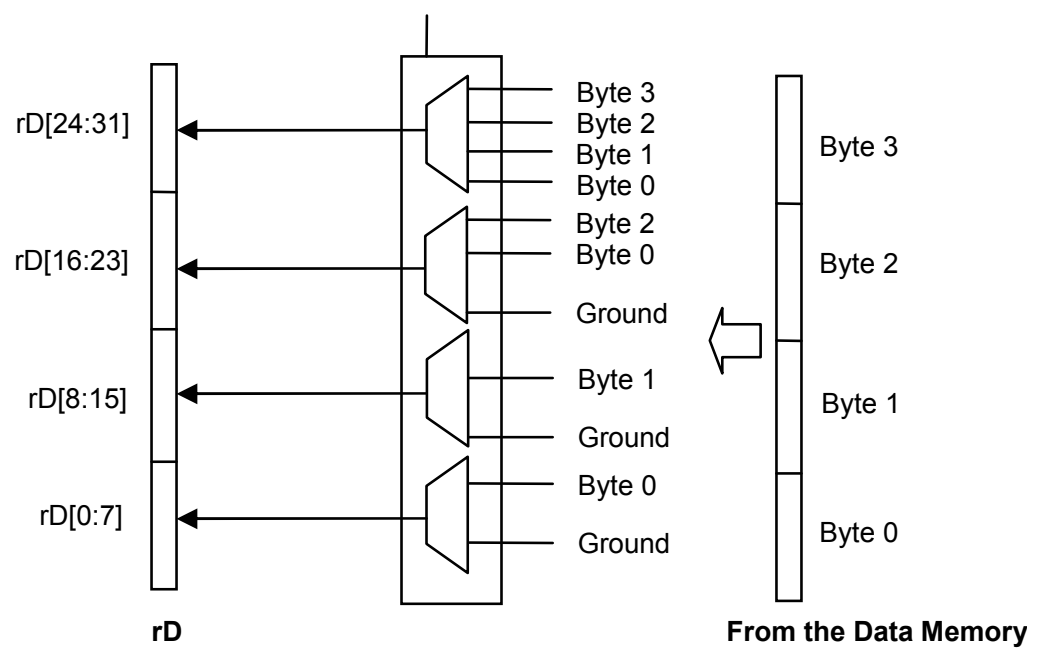

Figure 3.12: Read Data Steering

\subsubsection{Fifth Stage - Result Write Back}

Once the value of the destination operand is obtained, the write back stage updates the corresponding register in the register file as shown in Figure 3.5. The write port of the register file is clocked at the positive clock edge, so the current instruction in the decoding stage will read the up-to-date value if it tries to read the same register being updated by the write back stage. This resolves one of potential RAW hazards as explained in the following section. 


\subsubsection{Hazard Resolution}

In order to overcome RAW data hazards (see section 2.6) due to the overlapped execution of instructions, the base processor uses different mechanisms such as data forwarding and pipeline stalling whenever possible.

RAW hazards occur whenever consecutive dependent (the destination register of an instruction is used as one of source registers of a following instruction) instructions are in progress as shown in

Table 3.8. For example, the "OR" instruction needs R2, calculated by "ADD" instruction during the execution stage. However, the results of the "ADD" instruction are still in Memory access stage. In order to overcome this hazard, data is forwarded from the memory access stage to the execution stage.

Table 3.8: RAW Hazards in Progress

\begin{tabular}{|l|c|c|c|c|c|}
\hline & Fetch & Decode & Execute & Mem. Acess & Write Back \\
\hline Instruction & BEQ R5,R2 & ADD R6,R2,R8 & OR R5,R2,R3 & ADD R2,R3,R4 & \\
\hline
\end{tabular}

The techniques used by the base processor to overcome these RAW hazards whenever the dependent instructions are in the execution stage and in the memory access stage are listed in Table 3.9 for generalized cases. The RAW hazards can also occur when the dependant instructions are at the write back stage and at the execution stage. This type of hazard is handled by forwarding the data from the write back stage to the execution stage. During the situations where two dependant instructions are at the decoding stage and at the write back stage, the RAW hazard still exists. This situation is resolved by clocking the write port of the register file at the positive edge. Since the pipeline is negative edge triggered, the dependent instruction at the decoding stage always gets the up-to-date value from the register file, when progresses to the execution stage. The data forwarding and stalling the appropriate stages are done by the hazard control unit. The inputs of the ALU are multiplexed with the feedback operands from the memory access and write back stages, so the hazard control unit can select the correct operand to overcome the pipeline hazards. 
Table 3.9: Hazard Resolution

\begin{tabular}{|c|l|l|}
\hline $\begin{array}{c}\text { Instruction type } \\
\text { stage mem. access }\end{array}$ & $\begin{array}{l}\text { Instruction } \\
\text { type at exe. } \\
\text { stage }\end{array}$ & Hazard resolution techniques used \\
\hline ALU operation & $\begin{array}{c}\text { ALU or branch } \\
\text { operation }\end{array}$ & Data forwarded from the memory access stage to the execution stage. \\
\hline $\begin{array}{c}\text { Memory } \\
\text { operation }\end{array}$ & $\begin{array}{c}\text { Memory } \\
\text { operation }\end{array}$ & $\begin{array}{l}\text { Execution stage stalled. } \\
\text { Data forwarded from the memory access stage to the execution stage. }\end{array}$ \\
\hline $\begin{array}{c}\text { Memory } \\
\text { operation }\end{array}$ & $\begin{array}{c}\text { ALU operation } \\
\text { Memory }\end{array}$ & $\begin{array}{l}\text { Execution stage stalled. } \\
\text { Data forwarded from the memory access stage to the execution stage. }\end{array}$ \\
\hline operation & operation & $\begin{array}{l}\text { Execution stage stalled. } \\
\text { Data forwarded from the memory access stage to the execution stage. }\end{array}$ \\
\hline
\end{tabular}

The hazard control unit continuously monitors the destination and source operands in each stage. Depending on the instruction type in each stage, the hazard control unit generates appropriate control signals for stalling and forwarding. Figure 3.13 shows the hazard control unit and the forwarding paths connected through the multiplexers to the inputs of the ALU. Overlapped execution of instructions due to pipelining also introduces control hazards. When a branch is resolved at the execution stage, and if it is taken, the instruction in the decoding stage continues to execute. This type of hazard is known as a control hazard, and two simple methods can be used to resolve this hazard. The first method is to flush the decoding stage, and the second method is to allow the instruction to complete. The latter method is known as "delay slots", where the instruction following the branch is always executed in the pipeline irrespective of the outcome of the branch. MicroBlaze architecture addresses this issue using both methods. Microblaze branch instructions have two variations where one format uses a delay slot and the other uses pipeline flushes. The base processor uses the same mechanism to overcome control hazards due to branch instructions in order to maintain the cycle accuracy with Microblaze. For the branch instruction without a delay slot, the decoding stage is flushed once the branch is resolved during the execution stage. For the branch instructions with a delay slot, the instruction in the decoding stage is allowed to complete. 


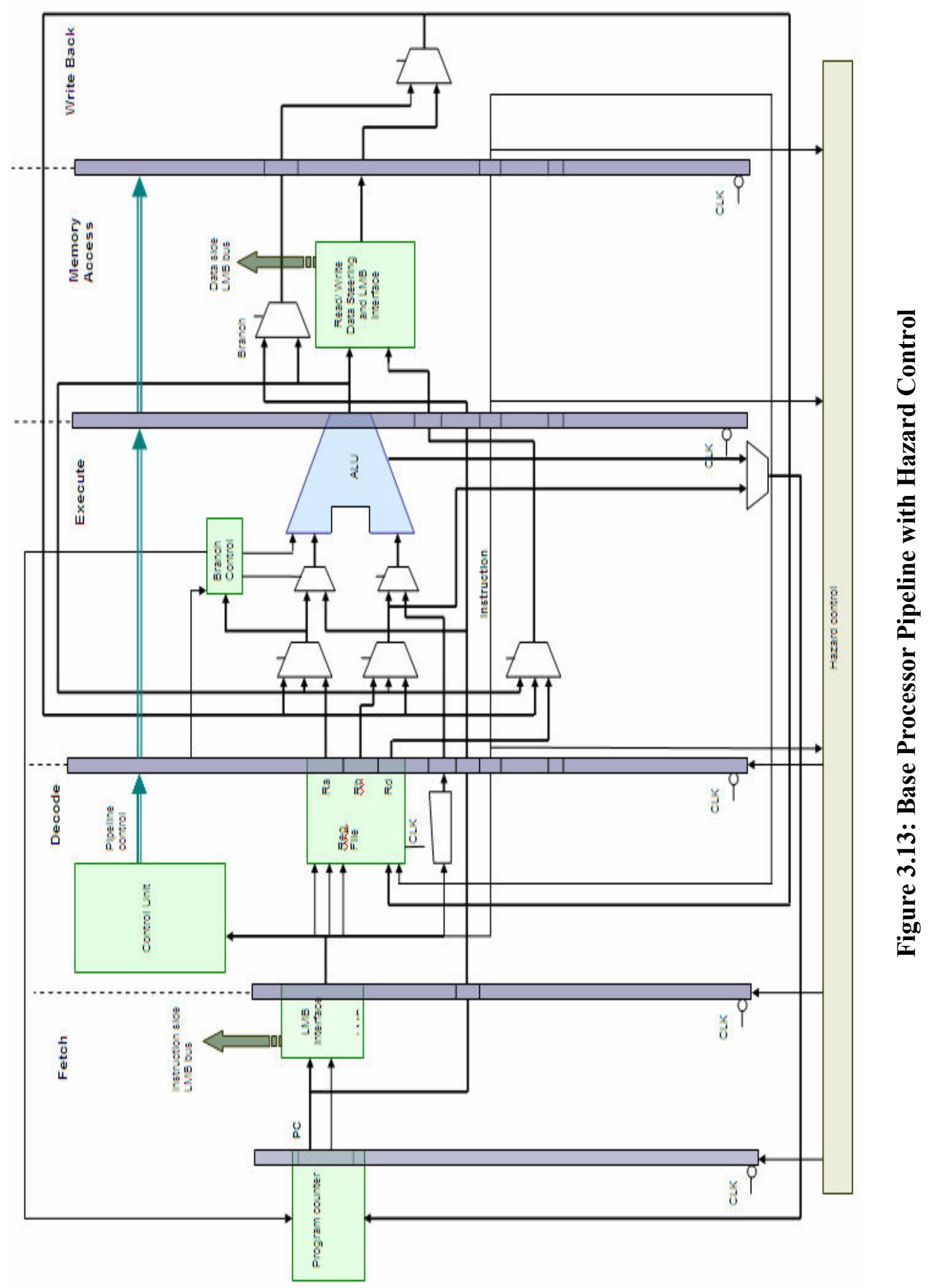




\subsection{Architecture of RTOS}

The second phase of this thesis extends the base processor with hardware RTOS support. This section examines the internal architecture of the software RTOSs, and the next section presents the concept and design of RTBlaze, a multithreaded processor with integrated RTOS functions in hardware.

RTOSs provide various services for the applications that run on top of the OS. The most important service provided by the RTOS is multithreading. The application programs can use multiple threads to perform various tasks concurrently. OSs use time multiplexing of each thread to achieve the concurrent behavior. Conventional RTOSs implement multithreading support in software on top of the basic instruction set architecture and interrupt handling mechanism of the processor.

Multithreading introduces different issues such as synchronization among threads. OSs provides a rich set of services to alleviate those issues due to multithreading. The application software can access these services through a special mechanism called system calls. Table 3.10 summarizes some basic system calls available in the Xilkernal [56].

Table 3.10: Basic System Calls Provided by a RTOS

\begin{tabular}{|l|l|}
\hline \multicolumn{1}{|c|}{ System call } & \multicolumn{1}{c|}{ Description } \\
\hline pthread_create & Creates a new thread \\
\hline pthread_exit & Kills the thread pointed by TID in Ra. \\
\hline register_int_handler & Creates an interrupt thread \\
\hline acknowledge_interrupt & Clears the interrupt. \\
\hline disable_interrupt & Kills the interrupt thread. \\
\hline sleep & Put the thread into sleep state for specific amount of time \\
\hline sem_init & Initialize a semaphore \\
\hline sem_wait & Wait operation on a semaphore \\
\hline sem_post & Post operation on a semaphore \\
\hline
\end{tabular}

The concept of multithreading is layered on top of a thread switching mechanism called context switching. Switching between the threads is used to emulate the 
concurrent execution of threads by time multiplexing. Whenever switching of a thread occurs, the states of all the architectural registers of the processor are saved in a block called the Task Control Block (TCB) in the memory. Execution of a new thread is resumed by loading all the architectural registers from the corresponding TCB.

In order to enable multithreading, threads need to be switched based on a particular policy. The scheduler is a software component which decides which thread to activate from a pool to execute. The selection is made using a particular preemptive scheduling policy implemented by the scheduler. Priority based preemptive scheduling is a commonly used policy, where each thread is assigned with a priority value. The scheduler gives the priority to the threads depending on this value. The system timer interrupt or a system call such as "sleep" or "create_thread" made by an application thread activates the scheduler and if required, a new thread in the ready pool is activated.

A thread has well defined states and the transition from one state to another occurs depending on several events. Figure 3.14 shows the state transition diagram of a thread. Once a thread is created, it is in the ready state. The scheduler selects the most suitable thread to run from the threads in the ready state. The thread currently selected by the scheduler uses the processor exclusively. This thread is in the running state. Only one thread can be in this state at any given time. If the currently running thread is suspended due to the activation of another thread, the current thread moves to the ready state. If the thread calls a system call "sleep", the state of the thread becomes waiting. This thread is waiting for a particular software timer to expire. When the timer expires, the state of the thread becomes ready. During the time the thread is in the waiting state, the scheduler schedules another thread making its state "running". If a thread tries to acquire a shared resource locked by another thread, the state of the thread becomes blocked. The state of the thread will transit to the ready state once the locked shared resource is released by some other thread. 


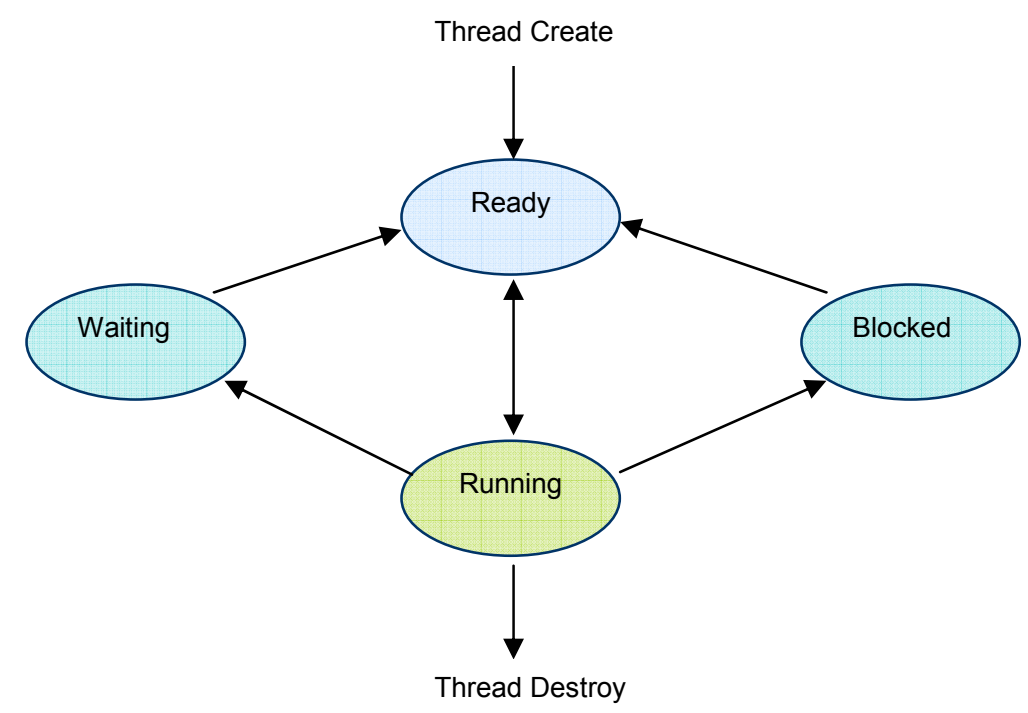

Figure 3.14: State Transition Diagram of a Thread

At the architectural level, each thread is identified by a unique identification number (TID), and each state of a thread is represented by a queue of TIDs. For example, all the threads in the ready state are enqueued in the ready queue. Figure 3.15 diagrammatically illustrates the context switching, scheduling, and the interrupt handling mechanisms of a RTOS.

Whenever application software creates a new thread, the thread ID is enqueued in the ready queue. If the RTOS implements a priority based scheduling algorithm, the thread is enqueued in the ready queue corresponding to the priority of the thread. If the state of the thread moves to another state, the thread is enqueued in the corresponding thread.

The hardware system contains a system timer which interrupts the processor in the pre-specified time intervals, mostly 10 milliseconds. This invokes a timer tick counter of the kernel and tracks the timing for the system. Once a thread makes a system call to put itself into the sleep state, the thread is enqueued in a timer queue and dequeued from the ready queue. Each timer tick interrupt scans the timer queue and decrements the timer counters of each thread. If it expires, the thread is re-enqueued in the ready queue and performs a rescheduling operation. 


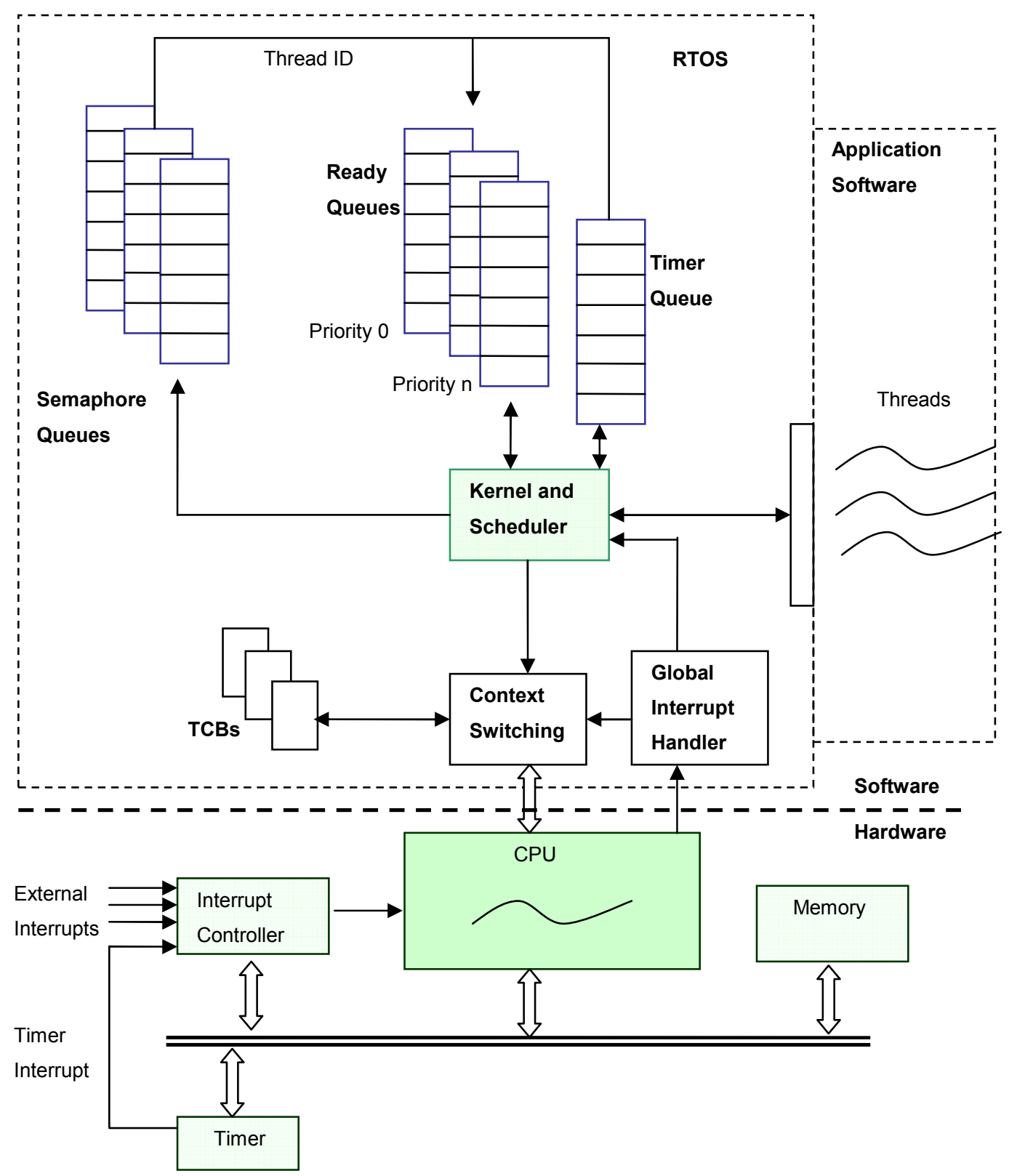

\section{Figure 3.15: Simplified Architecture of a RTOS Kernel Running On a SOPC}

In order to synchronize multiple threads, semaphores are provided by most OSs. A semaphore consists of a counter and a queue. Whenever a thread creates a new semaphore, the semaphore counter gets loaded with the requested semaphore value. A thread can wait for a given semaphore by performing a wait operation on the semaphore 
which results in decrementing its semaphore value. If the decremented value is less than zero, the thread which performed the wait operation is dequeued from the ready queue. Next, the thread is enqueued in the semaphore queue causing the thread to move to the blocked state from the running state. Any further wait operations on the semaphore cause the corresponding thread to be enqueued in the semaphore queue. When a thread performs a post operation on the semaphore, its value is incremented. If the result is equal to zero or greater, the highest priority thread in the semaphore wait queue is dequeued and enqueued in the ready queue.

Software RTOSs use layered interrupt handling mechanism. General purpose processors have single hardware interrupt and external interrupt controller [57] which are used to support multiple interrupt request lines (IRQs). OSs install a global handler for the main interrupt to resolve the correct ID of the external interrupt by accessing the external interrupt controller. Once resolved, the global handler calls the corresponding interrupt service routing in the application space. Some RTOSs use a mechanism to delegate the task of interrupt handling to application level threads called interrupt service threads (ISTs). In this case, the global interrupt handler enqueues the IST waiting for the corresponding IRQ in the ready queue, enabling the system to respond to further IRQs about to occur.

In the context of real-time systems, the software implementations of multithreading incur considerable overhead and contribute to increased nondeterministic behavior of the system as well as slower response. The layered approach to resolve the interrupt IDs introduces substantial interrupt latency. Most software mechanisms have the feature of the paralizability and are good candidates for direct hardware level implementation. For example, the priority based scheduler can be implemented by a priority decoder. The next section describes the design of RTBlaze, which implements RTOS mechanisms in hardware. 


\subsection{Design of the RTBlaze}

The main goal of the second phase of the thesis is to migrate software mechanisms of software RTOS mentioned in the previous section into hardware in order to eliminate the overhead associated with software implementations. To accomplish this, the instruction set of the base processor is augmented with support for the following OS level operations.

- Multithreading (thread creation/deletion)

- Interrupt service threads

- Timers

- Semaphores

Table 3.11 summarizes the RTBlaze instructions related to the RTOS functions. The current RTBlaze prototype implements a lightweight version of basic system calls of a RTOS. The "FORK" and "KILL" instructions are used for thread creation and deletion. The interrupt mechanism is handled using the "IFORK", "IKILL" and "ICLEAR" instructions while semaphore functions are accessed using the "SEMWAIT", "SEMINIT" and "SEMPOST" instructions.

Instruction set extension for RTOS services is integrated to the base processor pipeline by modifying and extending the pipeline with support for following entities.

- Context switching

- Thread scheduling

- Timers

- Semaphores

- Interrupts 


\section{Table 3.11: RTBlaze Instructions for RTOS Services}

\begin{tabular}{|c|c|}
\hline Instruction & Description \\
\hline FORK Rd, Ra, Rb & $\begin{array}{l}\text { A new thread is created and placed in the ready queue. The value } \\
\text { of Ra is used as the thread ID. Thread ID itself represent the } \\
\text { priority of the thread being created. Rb points to the address of } \\
\text { the first instruction to be executed when the thread is in run mode. }\end{array}$ \\
\hline KILL Rd, Ra, & Kills the thread pointed by TID in Ra. \\
\hline SLEEP Rd,Ra & $\begin{array}{l}\text { Current thread is enqueued in the timer queue and is awakened } \\
\text { after the cycles specified by Ra. }\end{array}$ \\
\hline IFORK Rd, Ra, Rb & $\begin{array}{l}\text { A new thread is created and corresponding interrupt line is } \\
\text { enabled. The value of Ra is used as the interrupt ID. Interrupt ID } \\
\text { itself represent the priority of the thread being created. Rb points } \\
\text { to the address of the first instruction to be executed when the } \\
\text { interrupt is occurred. }\end{array}$ \\
\hline ICLEAR Rd,Ra, Rb & $\begin{array}{l}\text { Clears the interrupt. The value of Ra is used as the interrupt ID. } \\
\text { interrupt ID itself represents the priority of the thread being } \\
\text { created. Rb points to the address of the first instruction to be } \\
\text { executed when the interrupt occurs. }\end{array}$ \\
\hline IKILL Rd,Ra & Kills the interrupt thread pointed by TID in Ra. \\
\hline SEMINIT Rd,Ra,Rb & $\begin{array}{l}\text { Initialize the count value of the semaphore identified by Ra. The } \\
\text { initial value of the semaphore is set to Rb. }\end{array}$ \\
\hline SEMWAIT Rd,Ra & $\begin{array}{l}\text { Increments the count value of the semaphore specified in Ra. If } \\
\text { the result is positive or zero the highest priority thread dequeued } \\
\text { from the semaphore wait queue and placed in the ready queue. }\end{array}$ \\
\hline SEMPOST Rd,Ra & $\begin{array}{l}\text { Decrement the count value of the semaphore by } 1 . \text { If the result is } \\
\text { smaller than zero, the current thread is dequeued from the ready } \\
\text { queue and placed in the corresponding semaphore wait queue. } \\
\text { Semaphore ID is specified in Ra. }\end{array}$ \\
\hline
\end{tabular}

Figure 3.16 shows the base processor with architectural extensions for RTOS support. In comparison with the software RTOSs, the operation is conceptually the same, but realized in hardware and operates in a parallel fashion. Hardware based mechanism for context switching is realized using the concept of multithreading. The base pipeline is extended with support for multithreading by replicating all the architectural registers for each thread and propagating a unique thread ID to identify the correct register space (or the context) with each instruction. The scheduler module implements a static priority 
based preemptive scheduler in hardware. Depending on the priority, the scheduler decides the next ready thread to run and propagates the selected thread ID to the multithreaded pipeline in order to switch to the context of the newly selected thread. Timers are implemented as independent hardware counters, thus, processor intervention for scanning the timer queues and counting down is not required. The semaphore module also performs all the operations in hardware in a parallel fashion. RTBlaze implements fast interrupt handling mechanism by directly mapping the ISTs to the hardware threads. This introduces another possible state for the RTBlaze threads as shown in Figure 3.17. Once created with the "IFORK" instruction the IST is in the waiting-for-IRQ state. Upon the occurrence of an external interrupt, the corresponding IST is enqueued in the ready queue, and the scheduler will select the IST depending on the priority.

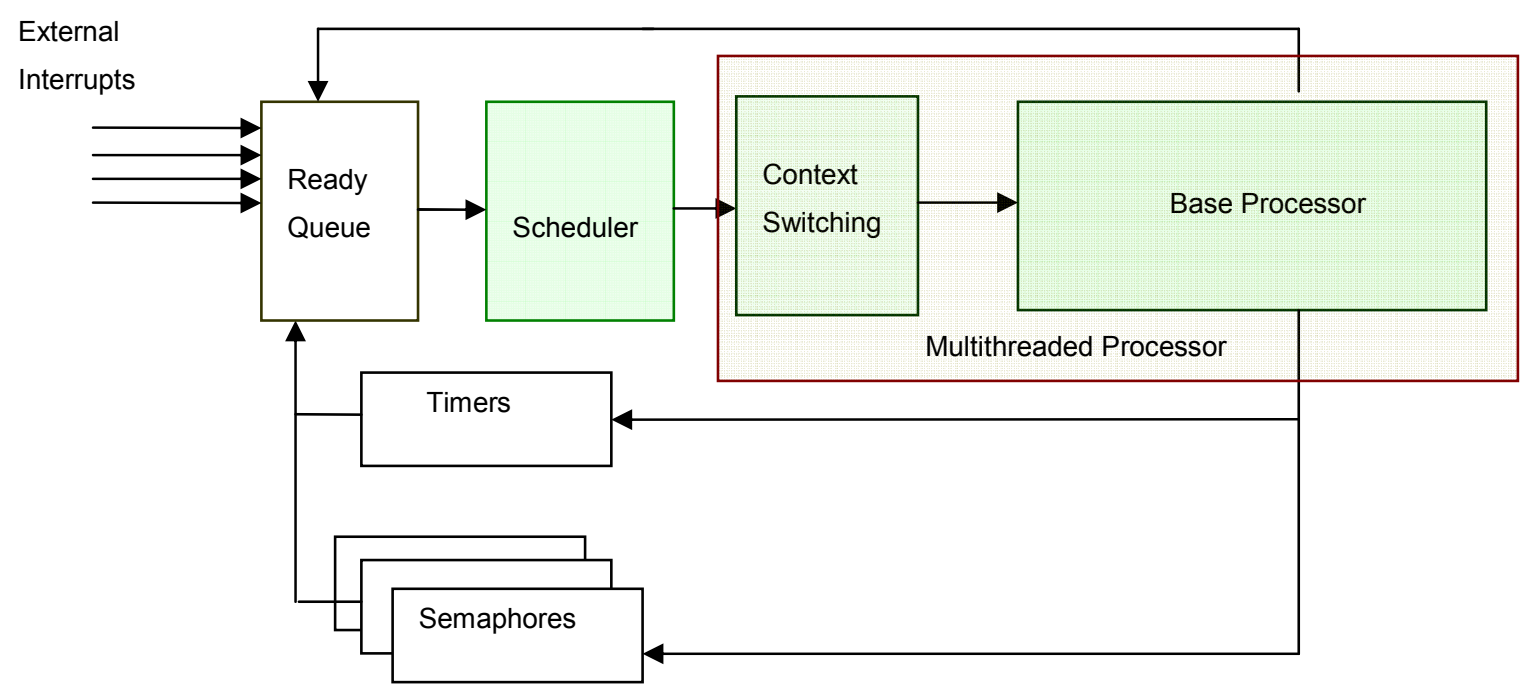

Figure 3.16: Base Processor with RTOS Extension 


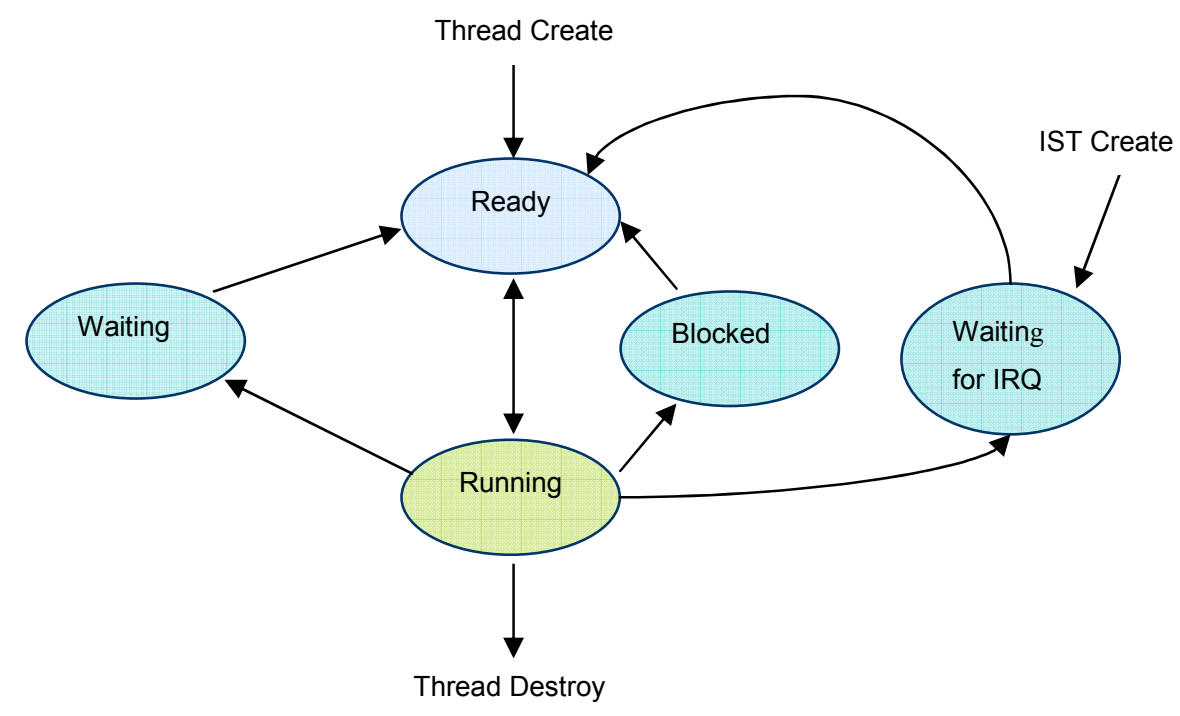

Figure 3.17: State Transition Diagram of an RTBlaze Thread

The modified pipeline of the base processor is shown in Figure 3.18. Multithreading support is added to the base processor pipeline by propagating the thread ID down the pipeline and replicating the register file for each thread. The selected thread ID is propagated through each pipeline stage with the instruction. This always guarantees that during the register file access, the correct register set is addressed. This is accomplished by appending the thread ID to each register address field of the instruction. The register write address is also qualified with the thread ID propagated to the writeback stage alone with the instruction. The writeback stage always writes to the correct register set even if the register file is being accessed by an instruction from another thread. Since the pipeline supports block interleaved multithreading, the hazard detection logic is always qualified with the thread IDs.

In order to enable multithreading, a thread scheduler with a program counter bank is introduced to the fetch stage. The scheduler decides the next thread to be executed, and the program counter bank stores all the distinct program counters associated with each thread. The scheduler supports a maximum of 16 threads with unique priority values ranging from 0 to 15 . The thread ID is also used as the priority of the thread. This concept simplifies the hardware, but the application programs have to track the currently-used thread IDs. TID zero is the lowest priority, and the thread associated with 


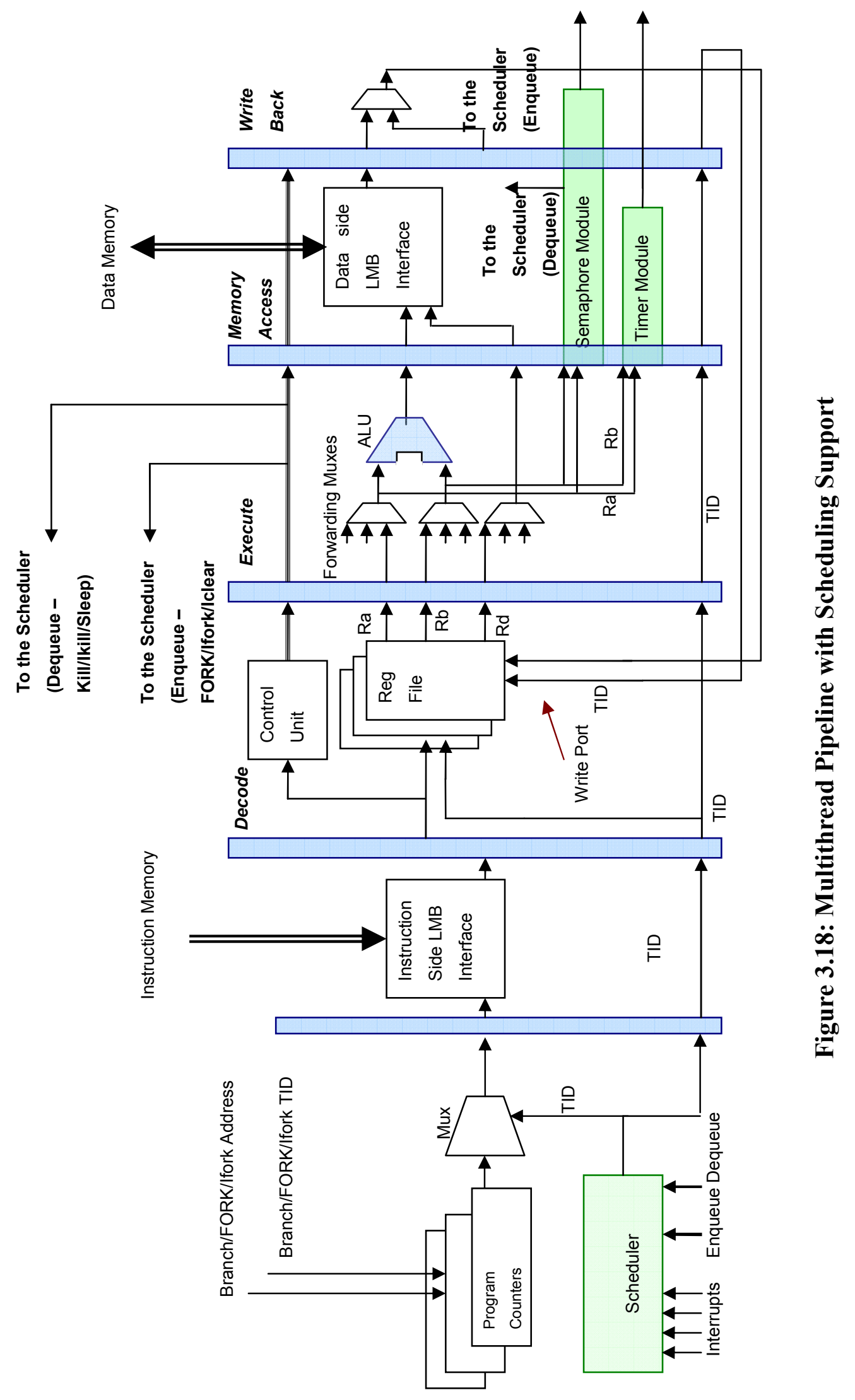


TID zero is automatically created upon reset and starts executing from address 0x00000000.

The timer module is aligned with the memory access stage of the pipeline. The semaphore module itself is pipelined and aligned with both the memory access and write back stage of the pipeline. Both timer and semaphore related instructions use register operands $(\mathrm{Ra}$ and $\mathrm{Rb}$ ) as their source values. These alignments help reuse existing forwarding paths to eliminate RAW hazards. The control unit is also modified to support decoding of those new instructions, and it generates the appropriate signals to manage the semaphore and timer modules. For example, whenever the decoding stage decodes a sleep instruction, it generates the control signals to dequeue the current thread from the ready queue. Appropriate control signals to load the timers with the corresponding value are also generated by the control unit.

Execution of this OS level instruction may cause preemption of the current thread and rescheduling of another thread. For example, if a "FORK" instruction is in progress in the pipeline, the execution stage resolves the operands of the "FORK" and enqueues the TID in the ready queue. If the newly created thread has the highest priority among other ready threads, the current thread gets preempted by this new thread. In this case, the instructions in the previous pipeline stages need to be flushed or defined as delay slots. RTBlaze uses later approach, since the simplicity inherent to the use of delay slots make the processor design straightforward.

The following sections present an elaborated discussion on the architectural extensions made to the base multithreaded processor in order to augment the instruction set with RTOS support.

\subsubsection{The Fetch Stage with Scheduling Support}

The scheduler contains a priority encoder attached to the ready queue. The ready queue is implemented in a parallel fashion. Therefore multiple elements can be updated simultaneously. The program counter bank is implemented using a dual port block RAM memory block. 


\subsubsection{Thread Scheduler}

Figure 3.19 shows the microarchitecture of the scheduler which implements the static priority based preemptive scheduling policy. It determines the TID of the next thread to be run. The scheduler contains a priority encoder with the ready queue flipflops. These flip-flops work as pipeline registers for the fetch stage. Each flip-flop represents each thread, and if the thread is ready, the flip-flop is set. Whenever the processor executes a "FORK" instruction, the ready bit related to that thread ID is set. The priority encoder selects the highest priority TID among all the active ready bits.

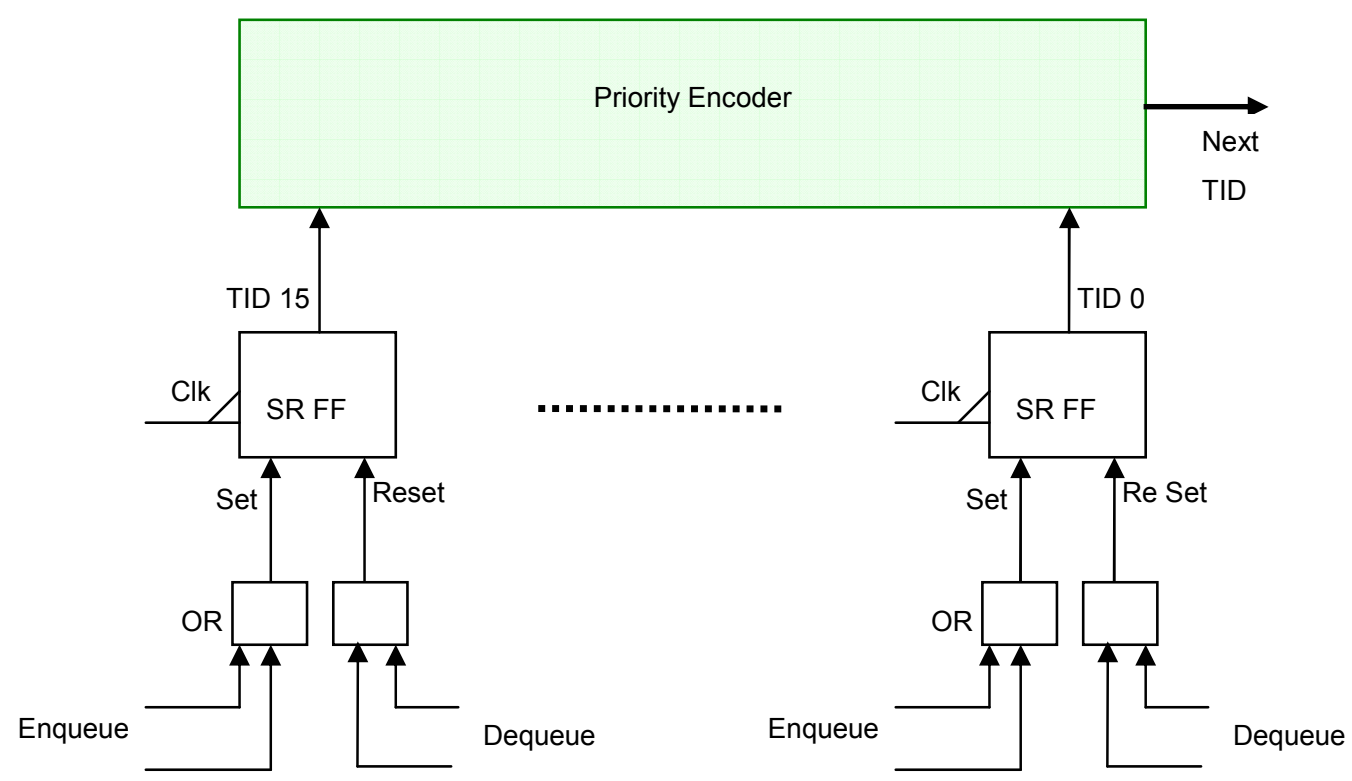

Figure 3.19: Microarchitecture of the Static Priority Based Thread Scheduler

\subsubsection{Program Counter Bank}

The fetch stage needs to handle all 16 program counters. The corresponding PC needs to be updated upon thread creation, taken branch, and normal sequential fetch conditions. Again, the PC of the currently scheduled threads needs to be read during each cycle. The modified fetch stage with scheduling support is shown in Figure 3.20. In order to simplify timing, all writes are performed on the negative edge of the clock and reads are performed asynchronously. This asynchronous read out allows the fetch to 
send the corresponding PC to the instruction side LMB in one cycle. To realize this PC bank, the distributed block RAMs with dual ported configuration with an asynchronous read port is used [58].

The write port of the PC RAM is multiplexed between the fetch stage and the execution stage, since the PCs in the block RAM need to be updated by either the fetch stage itself or the execution stage. When a FORK or branch instruction is in progress, the branch or FORK address is routed to the write port of the PC RAM through the multiplexer. For the sequential instruction fetch, the write port is used to update the PC of the current thread to point to the next instruction by the fetch stage itself. The multiplexer is controlled by the control unit depending on the whether the scheduler needs to execute the next sequential instruction or a branch/fork instruction.

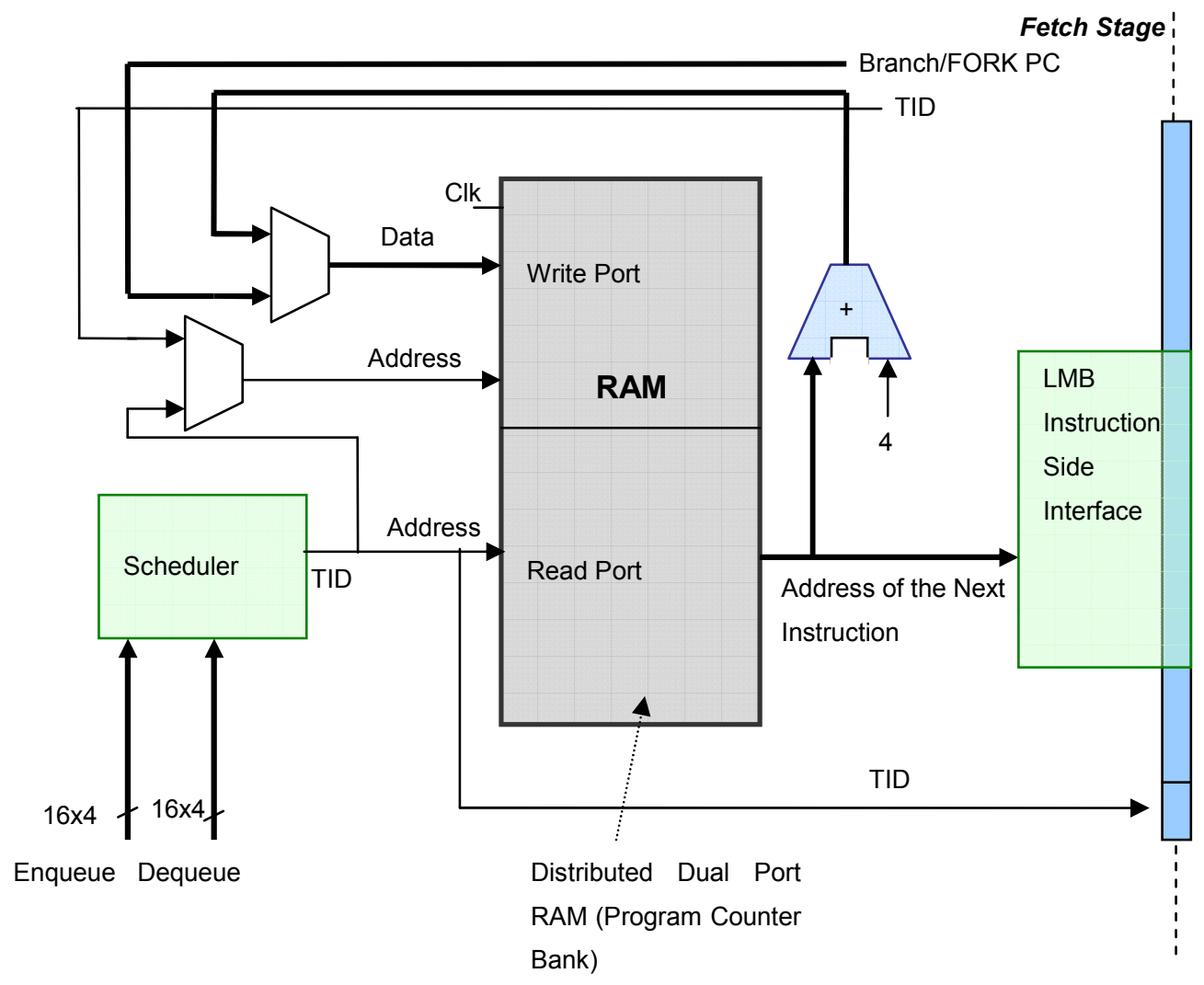

Figure 3.20: Modified Prefetch Stage with Scheduling Support 


\subsubsection{Support for Interrupt Service Threads}

This interrupt service mechanism is implemented using a simple extension to the scheduler microarchitecture as shown in Figure 3.21. Current RTBlaze configuration supports 4 hardware ISTs. These four threads are the same as the highest priority threads managed by the scheduler starting from TID 12 to 15, except that these ISTs are activated by external events. However, these four threads can be used as normal threads if not used as external interrupt threads.

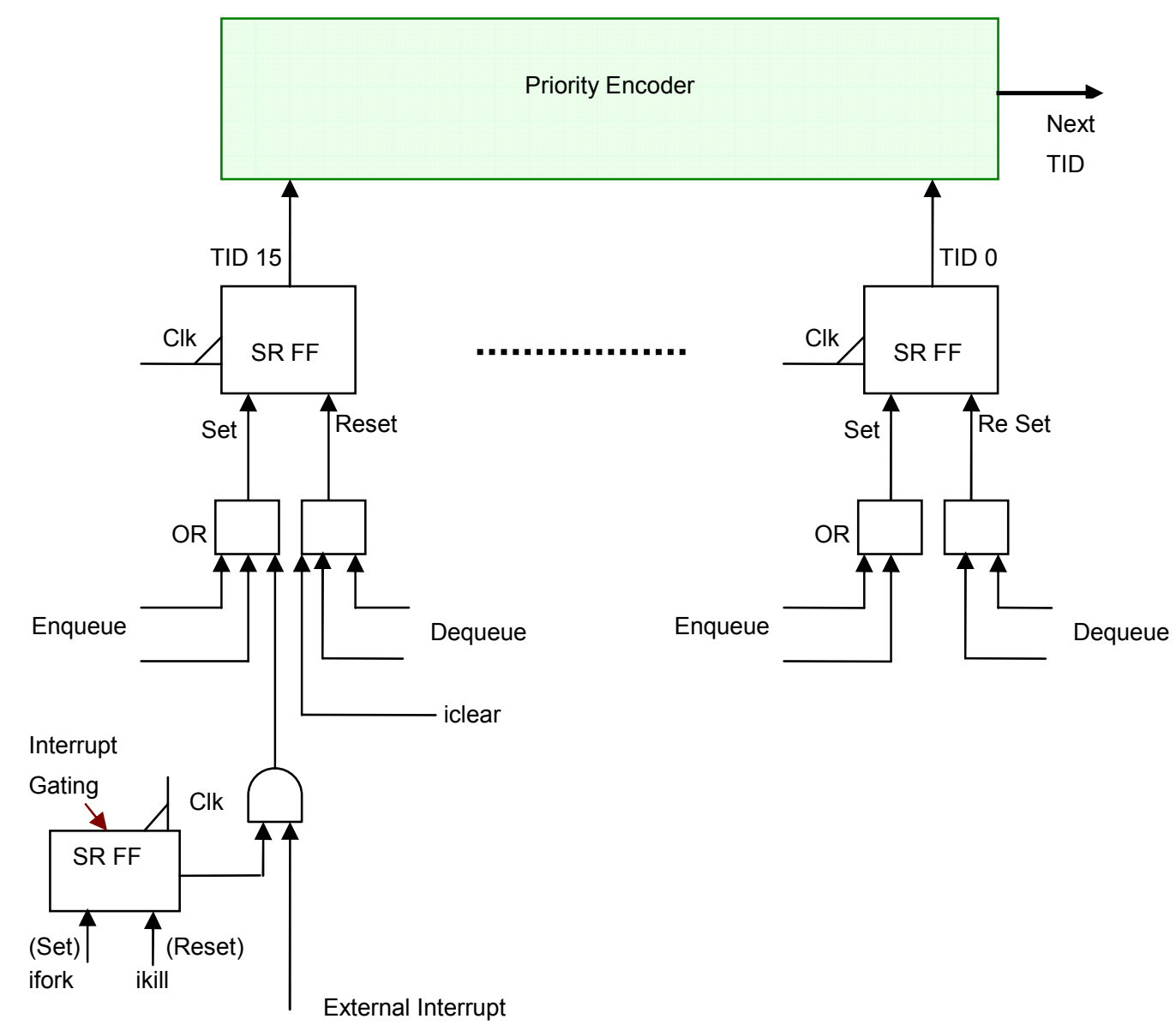

Figure 3.21: Extension of the Scheduler to Support Hardware ISTs.

The "IFORK" instruction sets the interrupt gating flip-flop which gates the external interrupt signal to the ready queue. Once serviced, the thread needs to call the "ICLEAR" instruction to clear the ready queue bit and restore the starting address of the 
IST. The "IKILL" instruction disables the interrupt by clearing the interrupt gating flip flop. This is equivalent to the interrupt disable functions of software RTOSs. Upon the reception of an interrupt, the corresponding hardware IST is scheduled during the next cycle if it is the highest priority thread currently in the ready state.

\subsubsection{Hardware Timers}

The RTBlaze support 15 hardware timers. The thread ID zero is not associated with a timer, since it is the idle thread and is supposed to be run whenever no other thread is ready. A thread can be put into the sleep state by executing the "SLEEP" instruction. The timers are implemented in direct mapped fashion with parallel execution. Figure 3.22 shows the microarchitecture of the direct mapped hardware timers. The timers do not consume any CPU cycles and the expiration is cycle accurate. This allows realizing high precision timers with the precision in a nanosecond scale provided that the thread requesting the timing is the highest priority thread currently active. Upon the execution of a "SLEEP" instruction by a thread, its timer is loaded with the number of cycles requested, and the thread is deactivated in the scheduler. The thread ID at the execution stage is used to enable the loading of the corresponding hardware timer through a decoder. Each element of the timer queue has its own down counter, and upon the expiration of the timer queue element the corresponding thread ID is reactivated in the ready queue. 


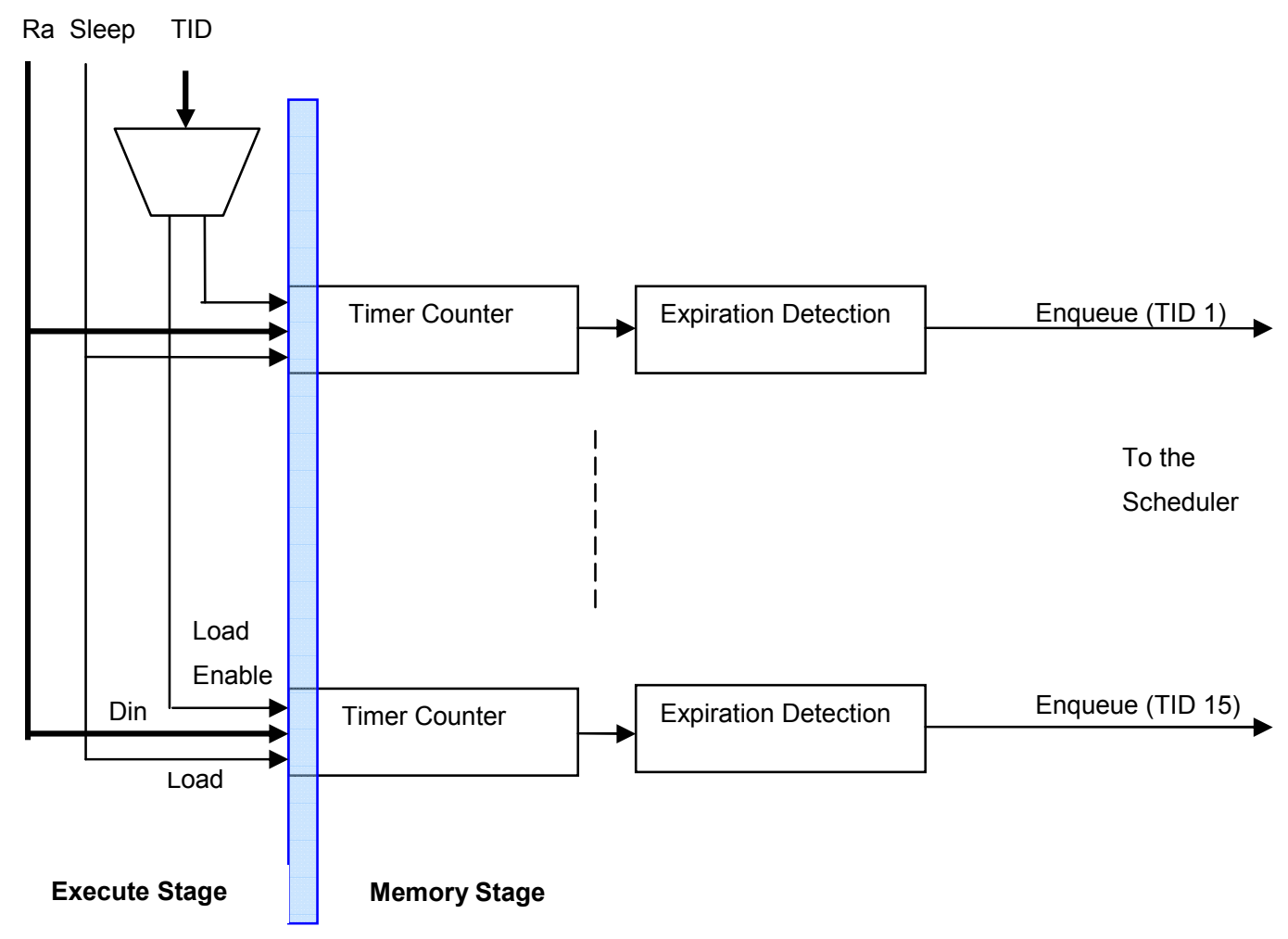

Figure 3.22: Direct Mapped Hardware Timers

\subsubsection{Hardware Semaphores}

When it comes to the multithreaded processors, they have the possibility to implement full hardware support for blocked synchronization schemes. Bradford, J.P. and S. Abraham [59] propose a blocking synchronization mechanism for multithreaded processors. The semaphore counters are stored in the memory and special semaphore handling instructions update those values according to the semaphore operations being performed. This scheme enables globally accessible semaphores in a context of a multiprocessor system. RTBlaze uses full hardware implementation of semaphores in order to provide an efficient mechanism for thread synchronization. The RTBlaze scheme is local to the processor and cannot be used to synchronize the thread in a different processor. However, unlike the scheme of Bradford, J.P. and S. Abraham, the RTBlaze implementation of semaphores results in zero overhead semaphore operations since this scheme does not use external memory for semaphore counters. 
RTBlaze implements four hardware semaphores. Each of these semaphores contains a four-element-deep priority queue in order to store waiting threads on the semaphore. Each semaphore has a unique ID ( 0 to 3 ) and can be used globally to identify the semaphore. The semaphore operations itself are pipelined and aligned with the main processor pipeline. Figure 3.23 shows the internal architecture of a single semaphore module. The decoder decodes the semaphore related instructions and controls the semaphore counters accordingly. For example, up, load and down signals are common to all four semaphores and those signals are generated by the control unit whenever a corresponding semaphore instruction is being executed. The enable signal activates the corresponding semaphore using the decoded value of Ra.

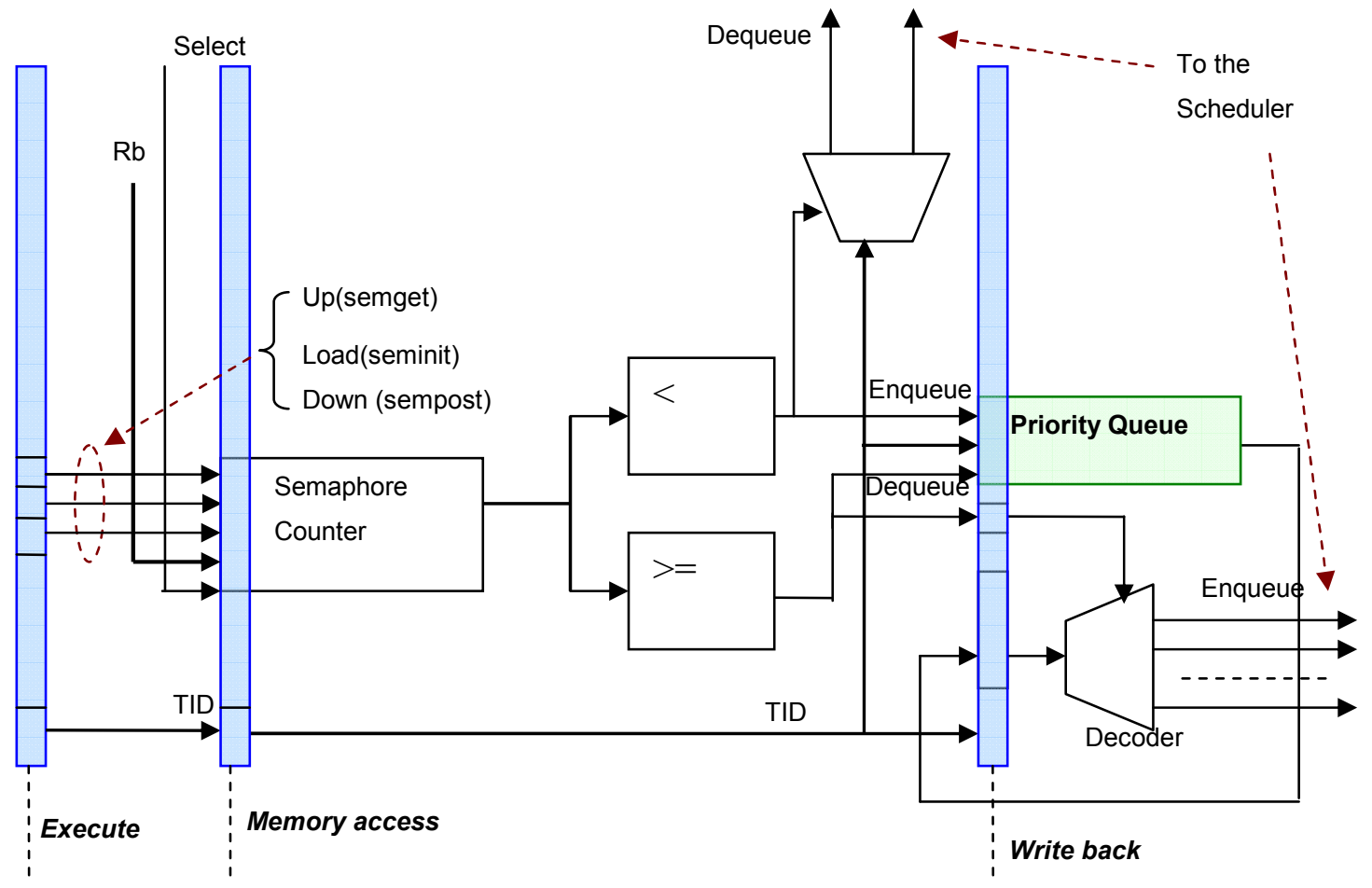

Figure 3.23: Microarchitecture of a Single Hardware Semaphore 
The semaphore operations are conceptually equivalent with the software based realizations. For example, the "SEMINIT" instruction loads the semaphore counter register identified by the value in Ra with the value in Rb. The "SEMWAIT" instruction decrements the semaphore counter value by one during the memory access stage. The comparison of the result is accomplished using dedicated comparators, and if the counter value is less than zero, the current TID is dequeued from the ready queue and enqueued in the priority queue. Both the enqueue and dequeue operations occur in write back stage. The "SEMPOST" operation increments the semaphore counter value, and if the value is zero or greater than zero, the top TID is enqueued from the priority queue and the corresponding thread is reactivated in the scheduler. Once enqueued in the semaphore wait queue, the items need to be removed according to the priority of the waiting threads. The highest priority thread needs to be removed first in order to prevent deadlocks. The priority queue [60] is implemented in hardware using the parallel version of the insertion sort algorithm. When the thread ID is inserted in the queue, it is placed in the corresponding location according to its value, and the queue items are automatically rearranged.

The priority queue is implemented using cascaded active queue elements. The microarchitecture of a single queue element is shown in Figure 3.24. Whenever a new thread ID needs to be inserted, the ID is transmitted to all the queue elements. Only one queue element latches the new entry depending on the comparison results. All other elements keep their values unchanged or shift down their values along the queue depending on the comparison results. This results in the implementation of the parallel version of the insertion sort algorithm. Therefore, an item can be inserted in the queue in a single cycle. The head of the queue always has the highest priority item which can be dequeued in a single cycle. 


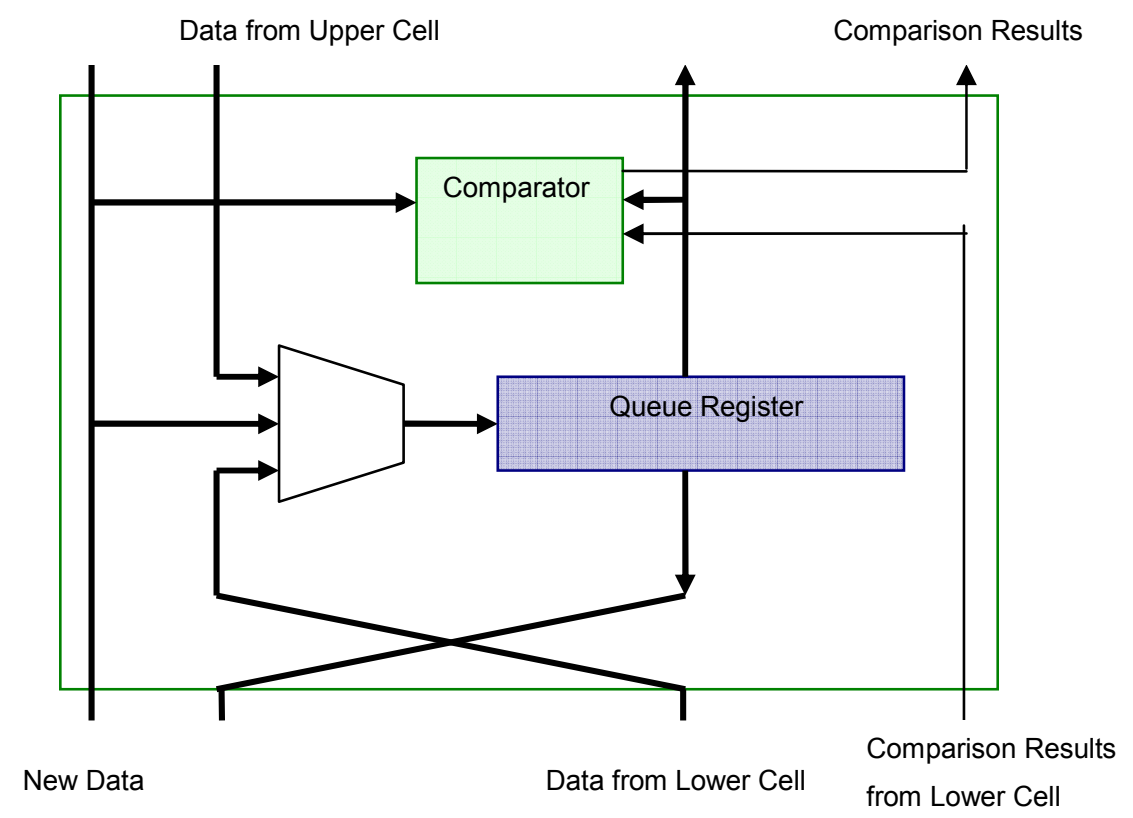

Figure 3.24: Microarchitecture of a Single Priority Queue Element

\subsection{Implementation and Performance of the RTBlaze}

Modern processor design is mostly an automated process where the main focus is on the architecture exploration to fit the processor for the targeted application domain. Architectural Definition Languages (ADL) [61] are used to accelerate the CPU design process. ADLs are used to specify the complete processor design, and these ADL specifications can be used to generate instruction set simulators, cycle accurate simulators and custom compilers. Some ADLs are smart enough to generate Register Transfer Level (RTL) level descriptions for synthesis of the complete processor. However, the resultant RTL descriptions are high level abstractions whereas the synthesis does not give an optimal design in the context of FPGAs. Therefore, VHDL is used to model the complete RTBlaze processor. When it comes to the domain of softcore processors, they need to be aligned to the underlying FPGA architecture to utilize different and appropriate hardware resources. For example, the multiplexers need to be properly mapped to the LUTs of the CLBs with minimum levels of logic. Behavioral 
level of design does not always give the optimum synthesis results even if advanced optimization features of the synthesis engine are enabled. Xilinx XST optimizations and structural level modeling is used throughout the design in order to maximally align the processor core with the Vertex II pro FPGA architecture. This helps to reduce the resource usage as well as the wastage while maximizing the maximum clock frequency. When targeted Vertex II pro, RTBlaze can be clocked up to a maximum of $119 \mathrm{MHz}$. 


\section{Chapter 4}

\section{Results}

The first section of this chapter presents the validation results of the base processor and the RTBlaze. The second part of this chapter compares the RTBlaze performance with some RTOS kernels. This chapter further investigates the chip area increase due to hardware RTOS attachment to the base processor.

\subsection{Simulation Results}

Simulation of the system is done using the Mentor Graphics ModelSim SE simulator. Simple assembly programs are used to simulate the basic functionality of the processor. The base processor is validated using random instructions from different categories, and the RTOS features of RTBlaze are validated using simple assembly programs. 


\subsubsection{Base Processor Validation}

Even though the current prototype of the base processor implements more than 70 instructions, not all of the instructions are validated. A subset of those instructions belongs to different categories is validated using some random instruction sequence. Table 4.1 shows the simple assembly program used to validate some of arithmetic and branch instructions.

Table 4.1: Assembly Program Used to Validate Arithmetic and Branch Instructions

\begin{tabular}{|l|l|l|l|l|}
\hline$\#$ & Memory Address & \multicolumn{1}{|c|}{ Contents } & \multicolumn{1}{c|}{ Instruction } & \multicolumn{1}{c|}{ Description } \\
\hline 1 & $0 x 00000020$ & 0x21800008 & ADDI R12,R0,0x8 & Load R12 with 0x8 \\
\hline 2 & $0 x 00000024$ & 0x 21A00002 & ADDI R13,R0,0x2 & Load R13 with 0x2 \\
\hline 3 & 0x00000028 & 0x058D6000 & RSUB R12,R13,R12 & R12 = R12-R13 \\
\hline 4 & 0x0000002C & 0xBC8CFFFC & BGTI R12,-0x4 & $\begin{array}{l}\text { Branch PC - 0x4 if R12 is greater } \\
\text { than 0 }\end{array}$ \\
\hline 5 & 0x00000030 & 0x21C00001 & ADDI R14,R0,0x1 & Load R14 with 0x1 \\
\hline 6 & 0x00000034 & 0x01EE6800 & ADD R15,R14,R13 & R15 = R14+ r 13 \\
\hline
\end{tabular}

First, two instructions load the corresponding registers with the immediate values provided with the instruction. The third instruction uses both R12 and R13 to execute, but the results are still not available in the register file. Therefore the hazard detection and control unit forward the results of the first and second instructions. The branch target is calculated using the immediate value provided with the instruction. The ALU is used to calculate this address with the special mode where the first operand (the program counter) is regarded as an unsigned value.

Whenever the branch occurs, the $5^{\text {th }}$ and $6^{\text {th }}$ instructions enter to the pipeline since the branch is resolved in the execution stage. Both these instructions are flushed at the decoding stage, so two bubbles (no-operations) are propagated in the pipeline. Figure 4.1, Figure 4.2, Figure 4.3, Figure 4.4, Table 4.3, and Table 4.2 illustrate the simulation results. 


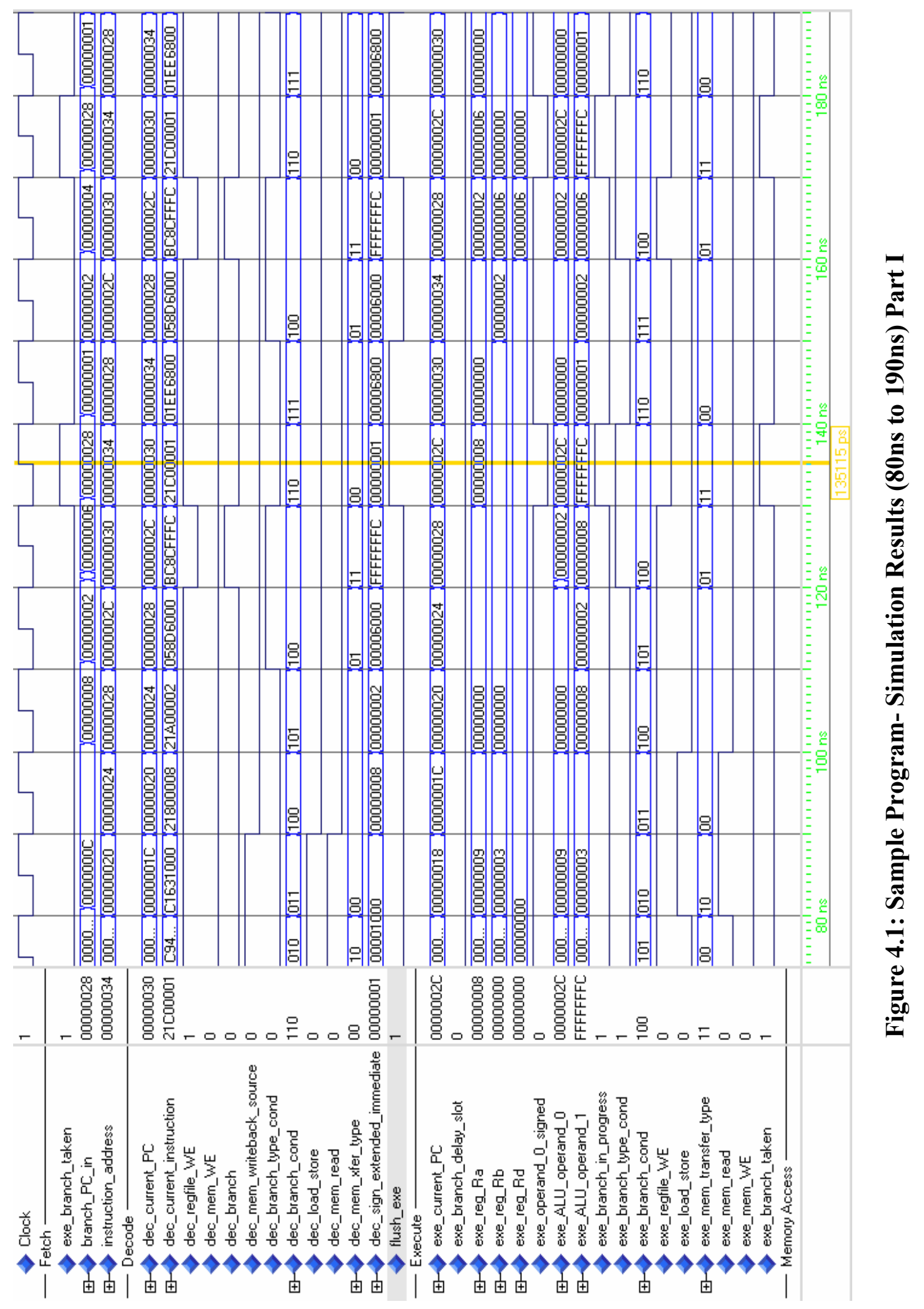




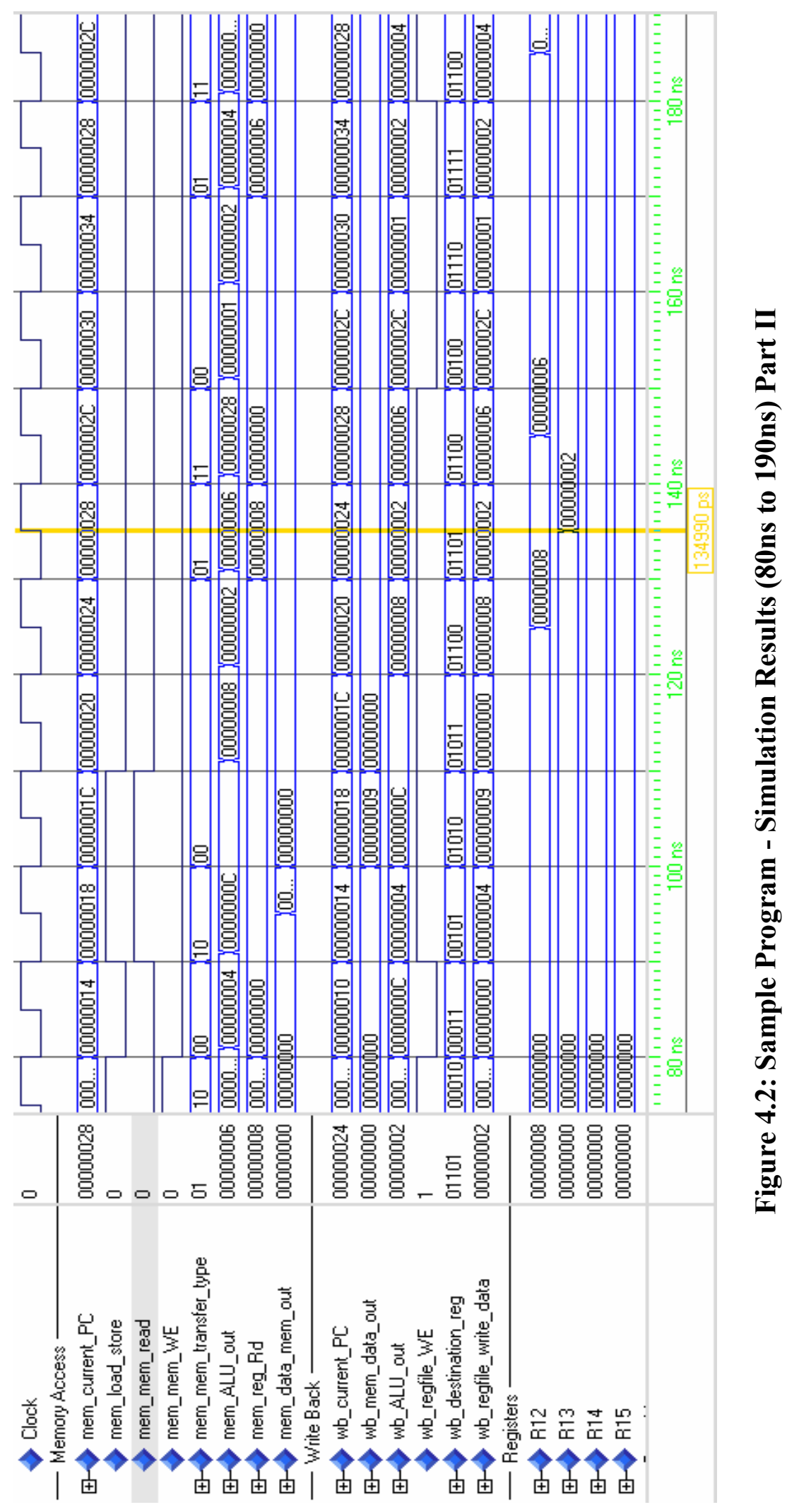




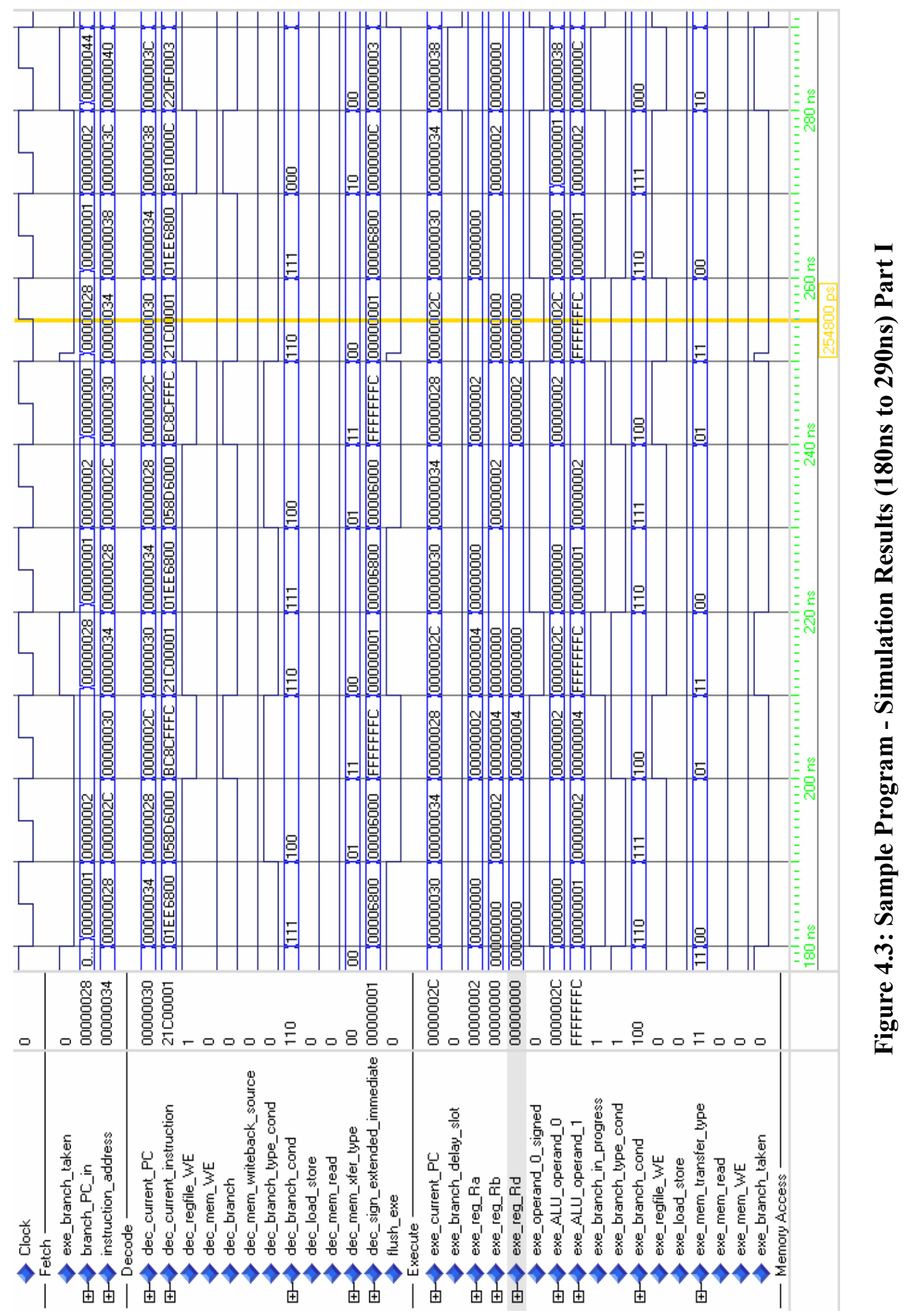




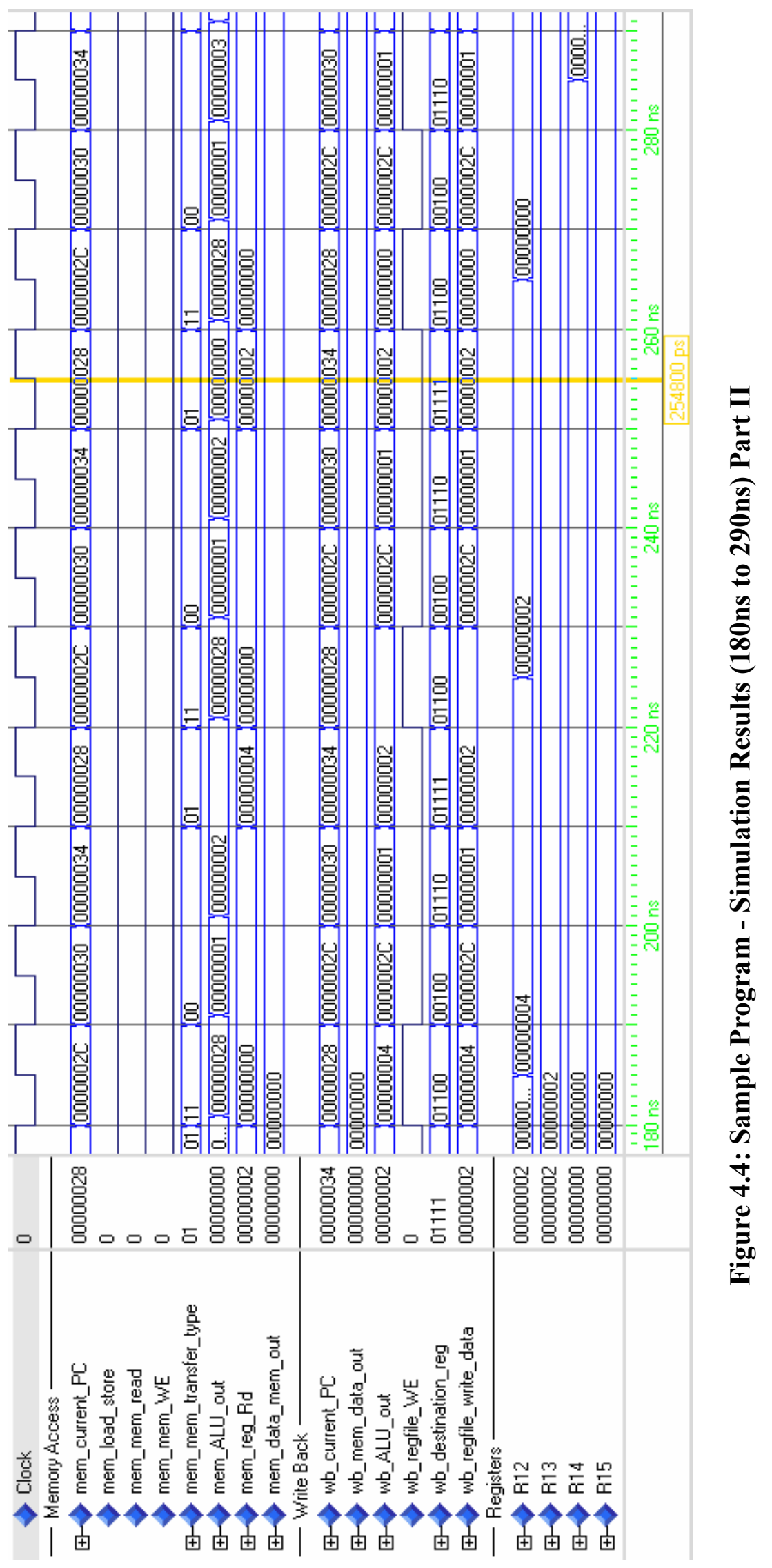




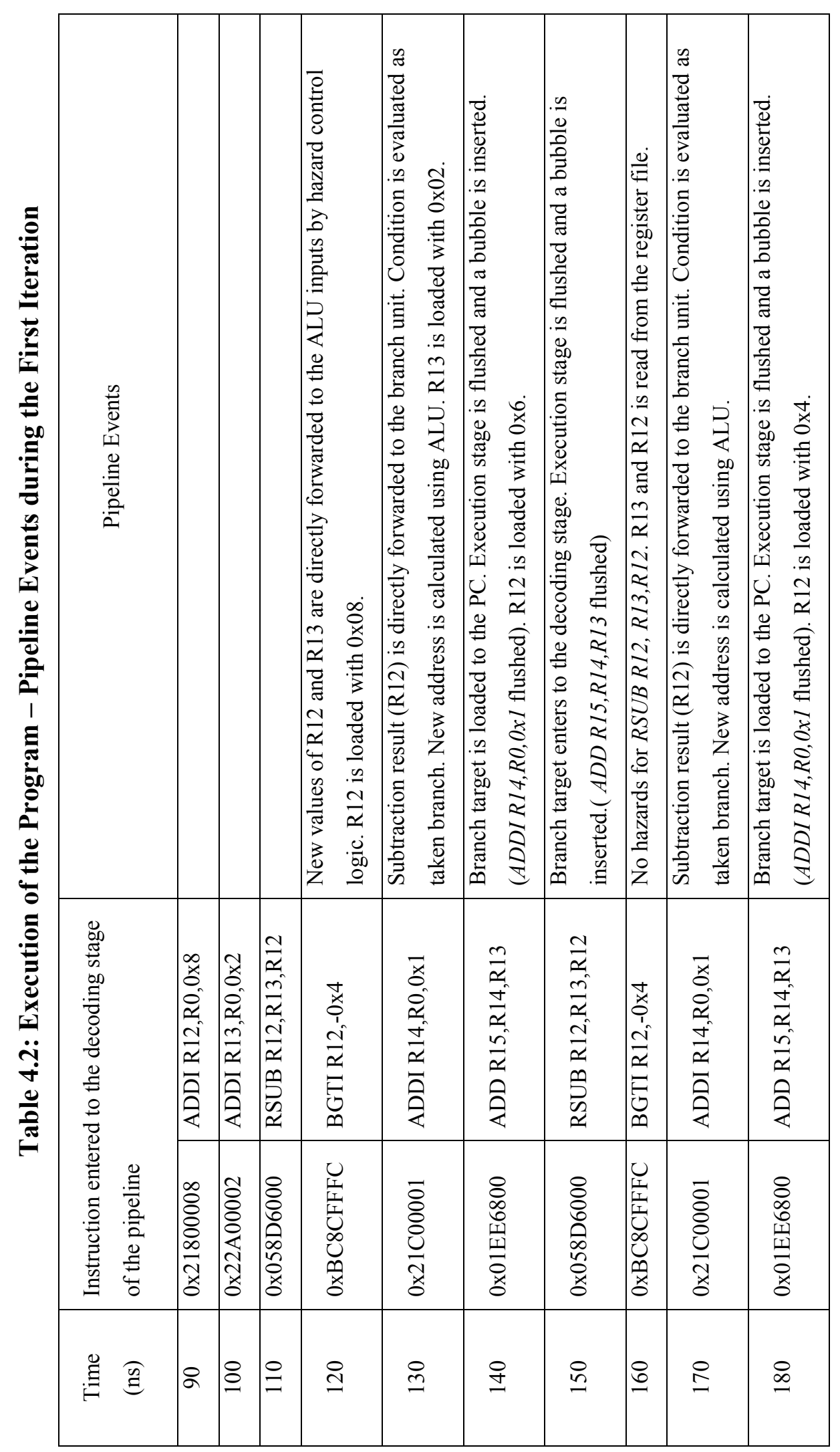




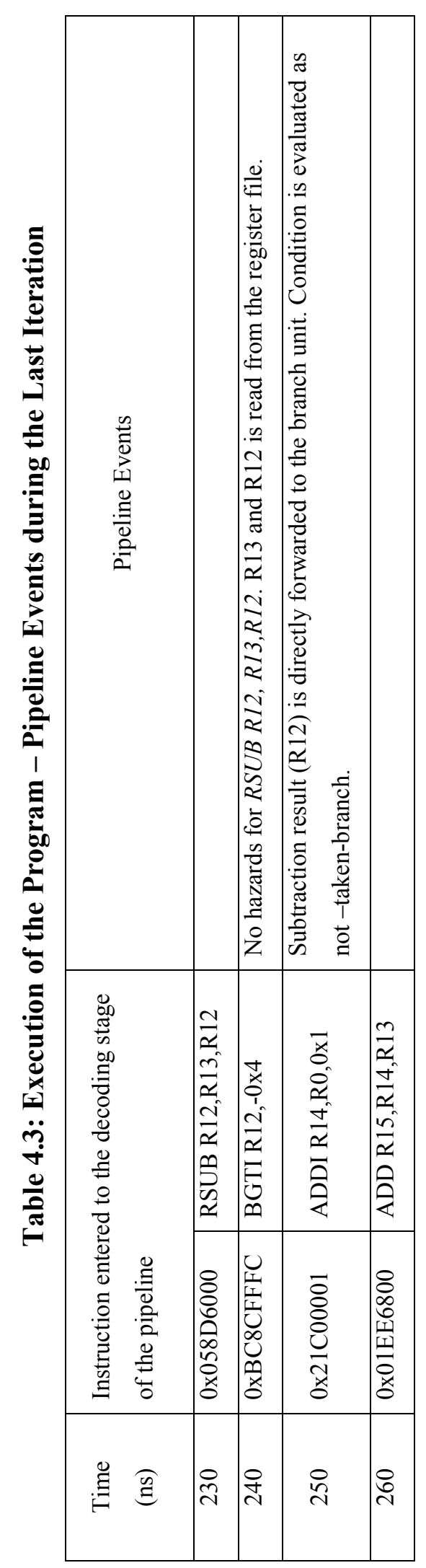


The Load/Store mechanism is validated using a simple assembly program which writes a value in a register to a specific memory location and reads it back. The simple assembly program provided in Table 4.4 is used for the simulation.

Table 4.4: Program to Validate Data Memory Access

\begin{tabular}{|c|l|c|l|l|}
\hline$\#$ & Memory Address & Contents & \multicolumn{1}{|c|}{ Instruction } & \multicolumn{1}{|c|}{ Description } \\
\hline 1 & 0x00000008 & 0x20600009 & ADDI R3,R0,0x9 & Load R3 with 0x9 \\
\hline 2 & 0x0000000C & 0x 20400003 & ADDI R2,R0,0x3 & Load R2 with 0x3 \\
\hline 3 & 0x00000010 & 0xD8631000 & SW R3,R3,R2 & Store R3 at Address (R3 + R2) \\
\hline 4 & 0x00000014 & 0x20A20001 & ADDI R5,R2,0x1 & Load R5 with 0x1 \\
\hline 5 & 0x00000018 & 0xC9431000 & LW R10,R3,R2 & Load R14 with Address (R3+R2) \\
\hline
\end{tabular}

The $3^{\text {rd }}$ instruction stores the contents of R3 at memory address R3 plus R2. The "SW" instruction simultaneously reads three operands and both R2 and R3 are forwarded by the hazard detection and control logic. The results are read back to R10 by the fifth instruction. Figure 4.5, Figure 4.6 and Table 4.5 illustrate the simulation results of the assembly program given in the Table 4.4 . 


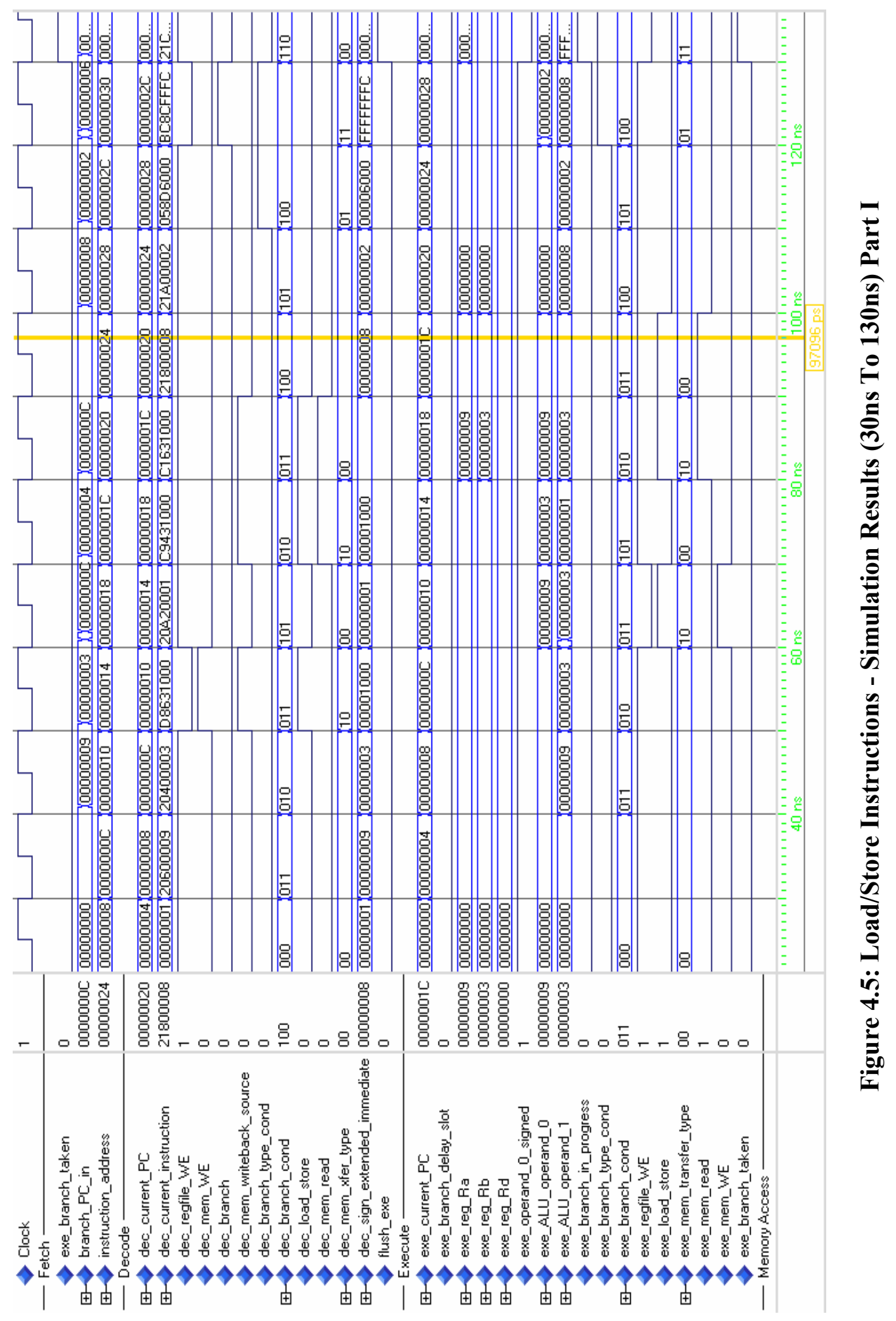




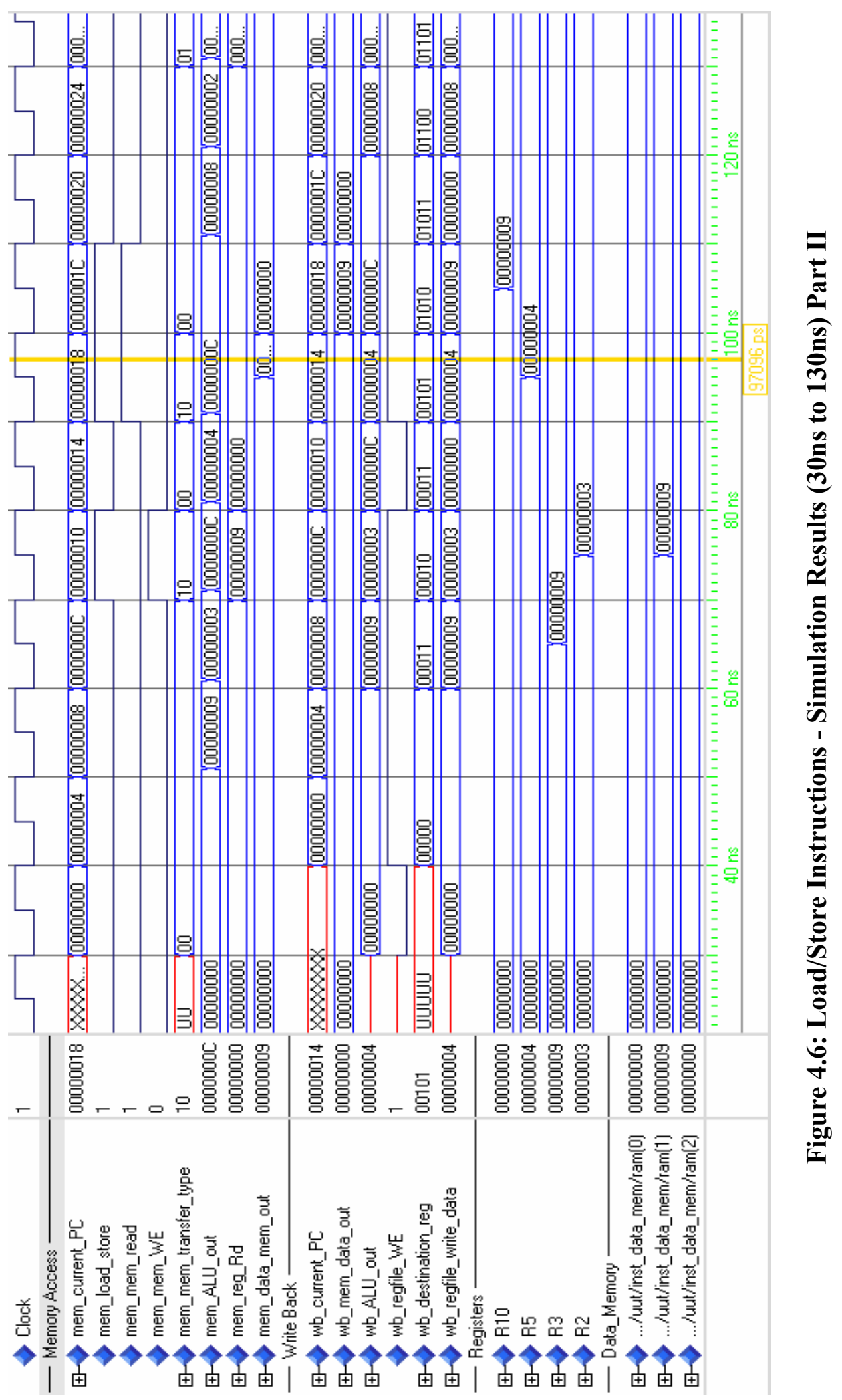




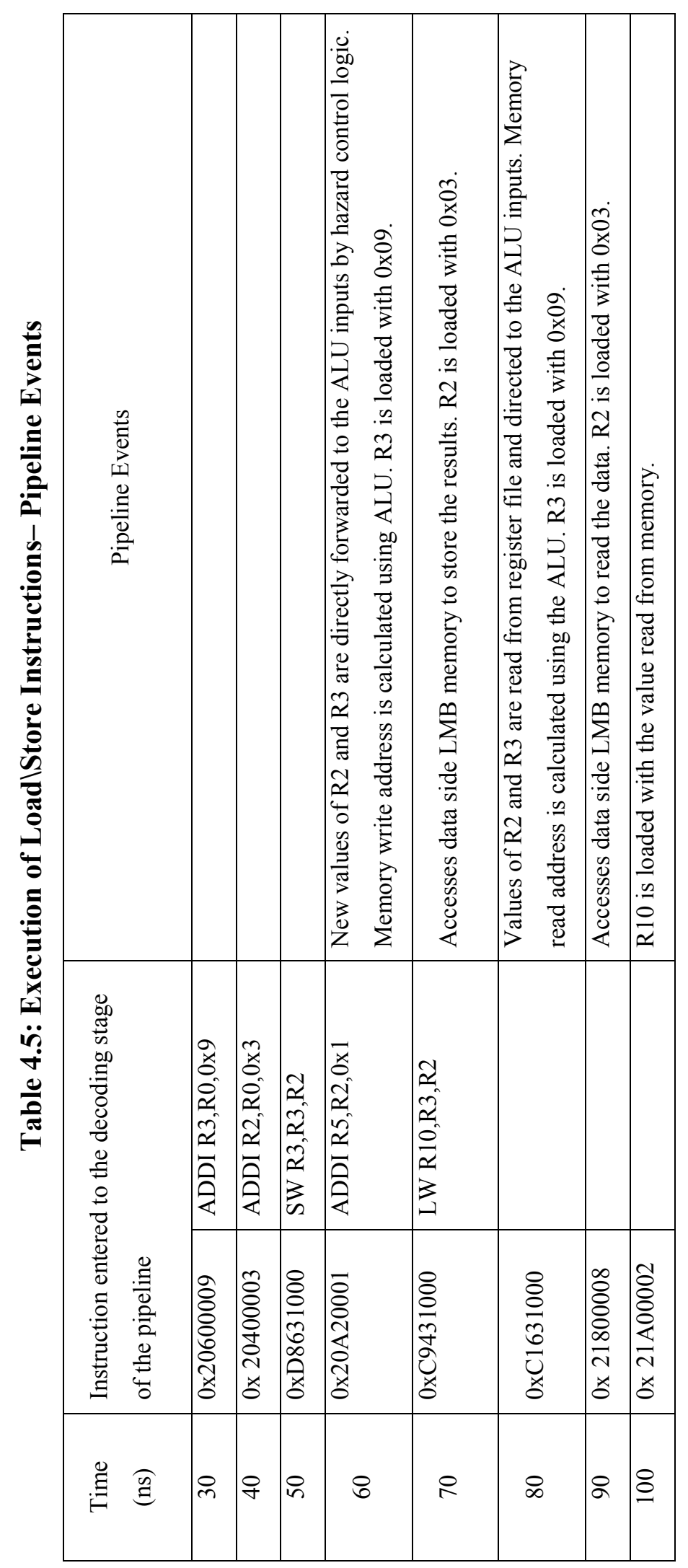




\subsubsection{RTBlaze Validation}

The following setup is used throughout the simulation and validation of the RTOS feature of RTBlaze.

- CPU runs at 100MHz clock

- One idle task is running (Thread ID zero)

Thread creation, timers, interrupt mechanism and semaphore based synchronization is verified in this section using simple assembly programs.

\subsubsection{Timing - Thread Create}

The idle thread executes "FORK" instruction to create a new thread. The priority of the thread is 2 , which causes the idle thread to be preempted and the newly created thread to start executing. The "FORK" instruction has two delay slots which always get executed. If independent instructions cannot be found, no-operations need to be used. The simulation results of thread creation assembly code listed in Table 4.6 is illustrated in Figure 4.7.

Table 4.6: Thread Creation

\begin{tabular}{|c|c|c|c|c|}
\hline \# & Memory Address & Contents & Instruction & Description \\
\hline 1 & 0x00000050 & $0 \times 2280006 \mathrm{C}$ & ADDI R20,R0,0x6c & $\begin{array}{l}\text { Starting address of the new } \\
\text { thread }\end{array}$ \\
\hline 2 & 0x00000054 & $0 \times 22 \mathrm{~A} 00002$ & ADDI R21,R0,0x2 & Priority of the new thread \\
\hline 3 & $0 x 00000058$ & $0 \times 5 F D 5 A 000$ & FORK R30,R21,R20 & Create a new thread \\
\hline 4 & 0x0000005C & $0 \times 22 \mathrm{C} 00004$ & & $\begin{array}{l}\text { FORK delay slot (always } \\
\text { executed) }\end{array}$ \\
\hline 5 & 0x00000060 & 0x22E00003 & & $\begin{array}{l}\text { FORK delay slot (always } \\
\text { executed) }\end{array}$ \\
\hline 6 & 0x0000006C & $0 \times 21800008$ & ADDI R12,R0,0x8 & $\begin{array}{l}\text { First instruction of the new } \\
\text { thread }\end{array}$ \\
\hline 7 & 0x00000070 & 0x 21A00002 & ADDI R13,R0,0x2 & \\
\hline
\end{tabular}




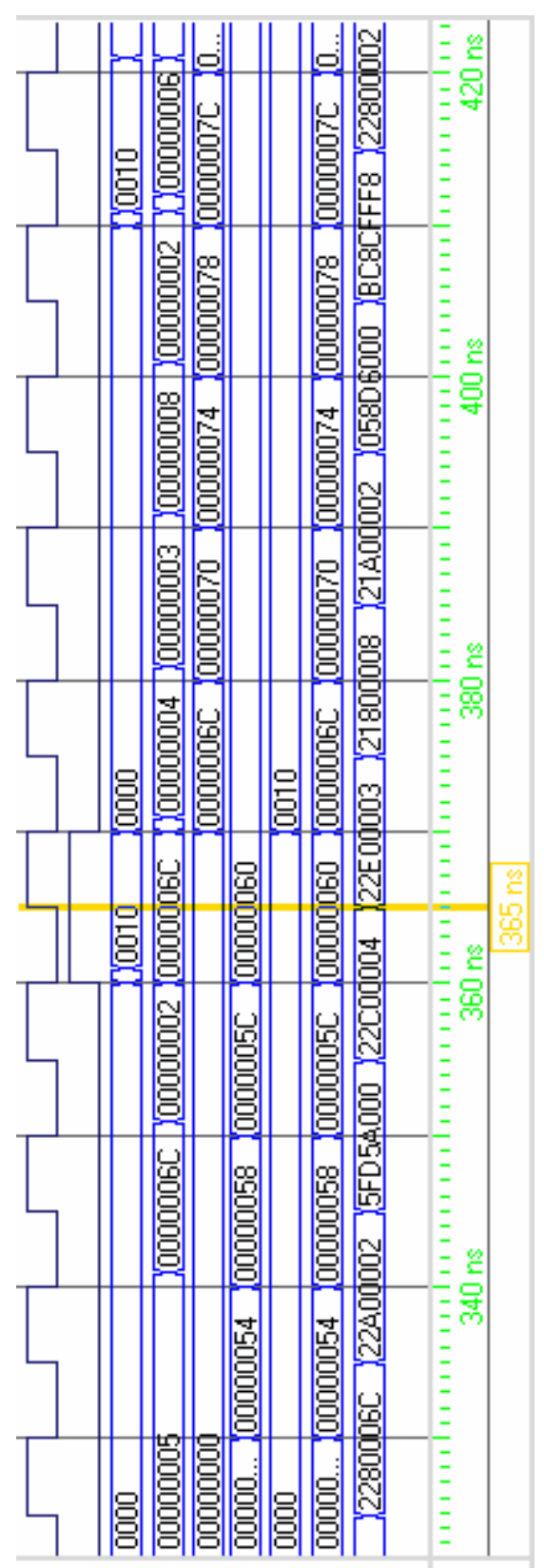

.

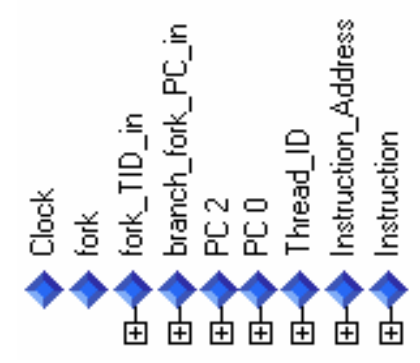

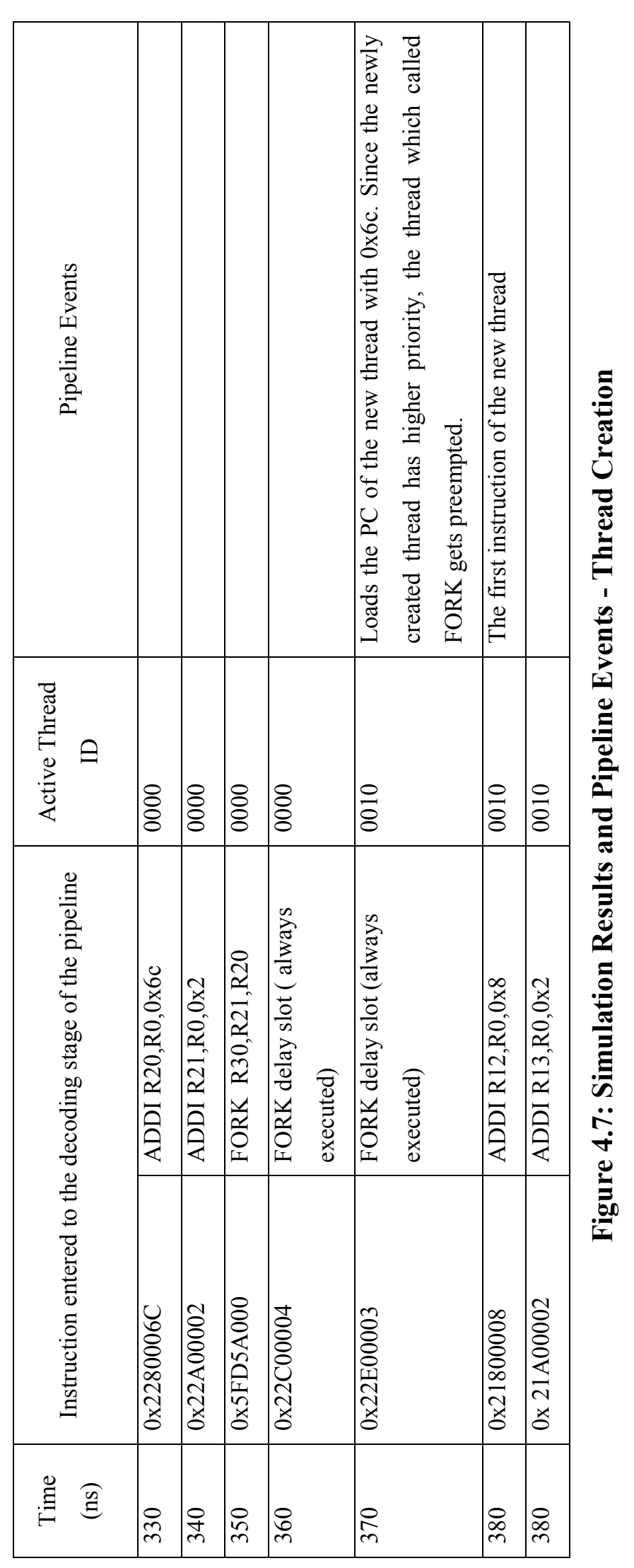

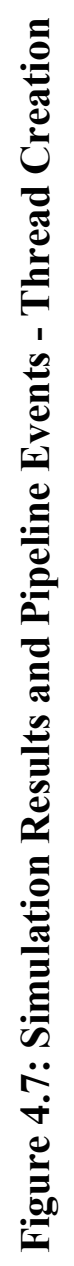

2 


\subsubsection{Timing - Thread Sleep}

The simple assembly code listed in Table 4.7 is used to validate the functionality of the timers. The thread ID 2 executes the "SLEEP" instruction requesting a 10 cycle sleep. Upon the execution of the "SLEEP" instruction, the thread ID 0 in the ready queue resumes the execution for 5 cycles, and is preempted again by the thread ID 2 when the timer expires. Figure 4.8 and Table 4.8 illustrate the simulation results.

Table 4.7: Thread Sleep

\begin{tabular}{|l|l|l|l|l|}
\hline$\#$ & \multicolumn{1}{|c|}{$\begin{array}{c}\text { Memory } \\
\text { Address }\end{array}$} & \multicolumn{1}{|c|}{ Contents } & \multicolumn{1}{|c|}{ Instruction } & \multicolumn{1}{|c|}{ Description } \\
\hline 1 & 0x0000008C & 0x 22A00005 & ADDI R21,R0,0x5 & Load R21 with 0x5 \\
\hline 2 & $0 x 00000090$ & 0x 5FD5A280 & SLEEP R30,R21 & Sleep 5 cycles \\
\hline 3 & $0 x 00000094$ & 0x 21C00001 & ADDI R14,R0,0x1 & Sleep delay slot \\
\hline 4 & 0x00000098 & 0x 22A00002 & ADDI R21,R0,0x2 & Sleep delay slot \\
\hline 5 & 0x0000009C & 0x 5FD5A380 & SEMWAIT R30,R21,R20 & \\
\hline & Idle thread & & & \\
\hline & 0x0000006C & 22E00003 & \\
\hline & 0x0000008C & 23000007 &
\end{tabular}




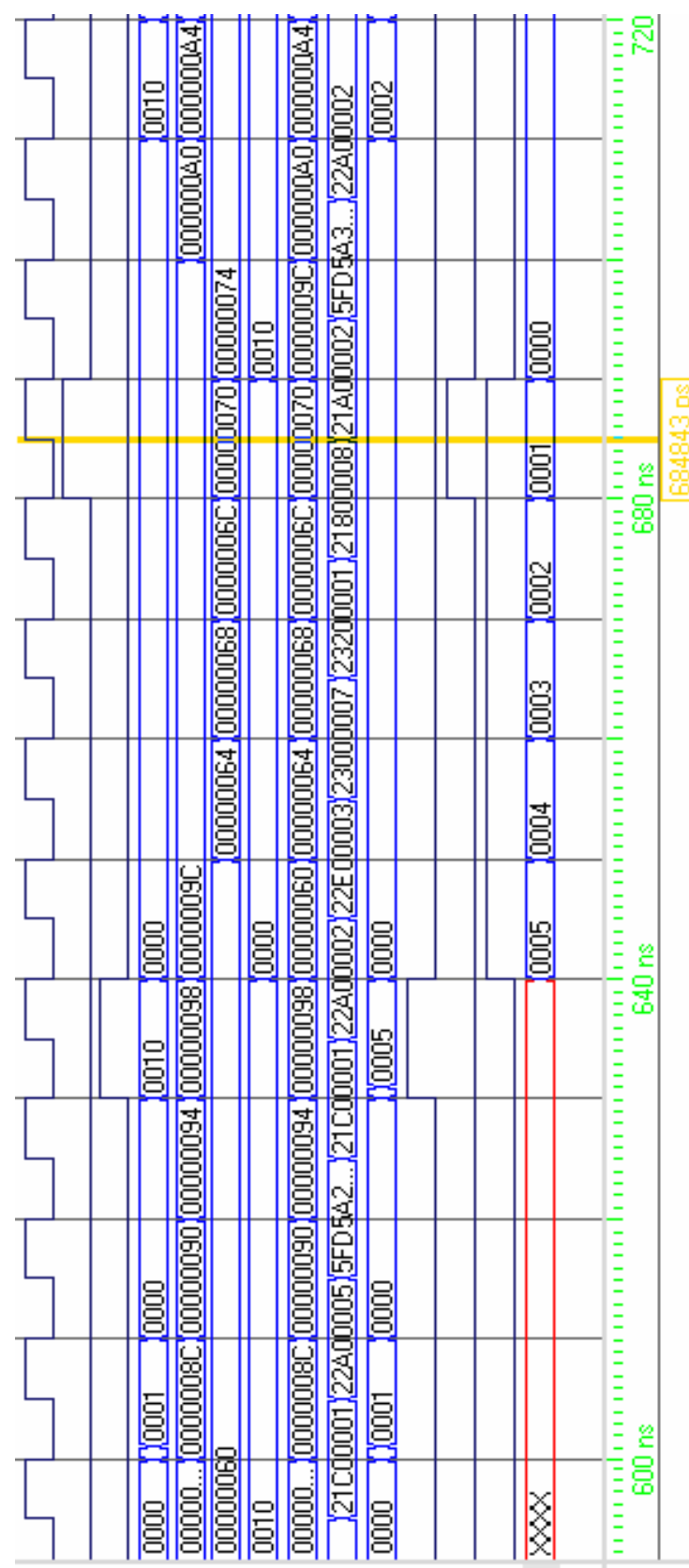

$\infty$

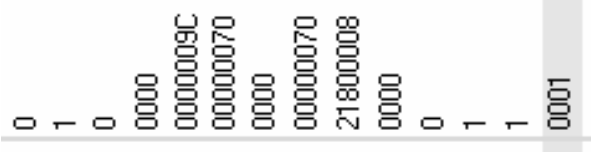

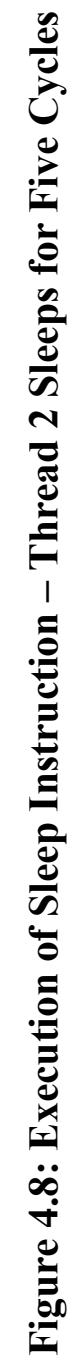

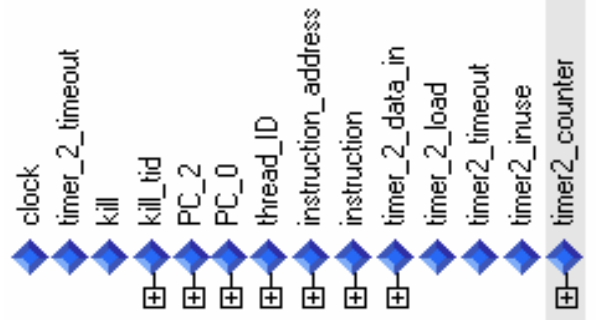




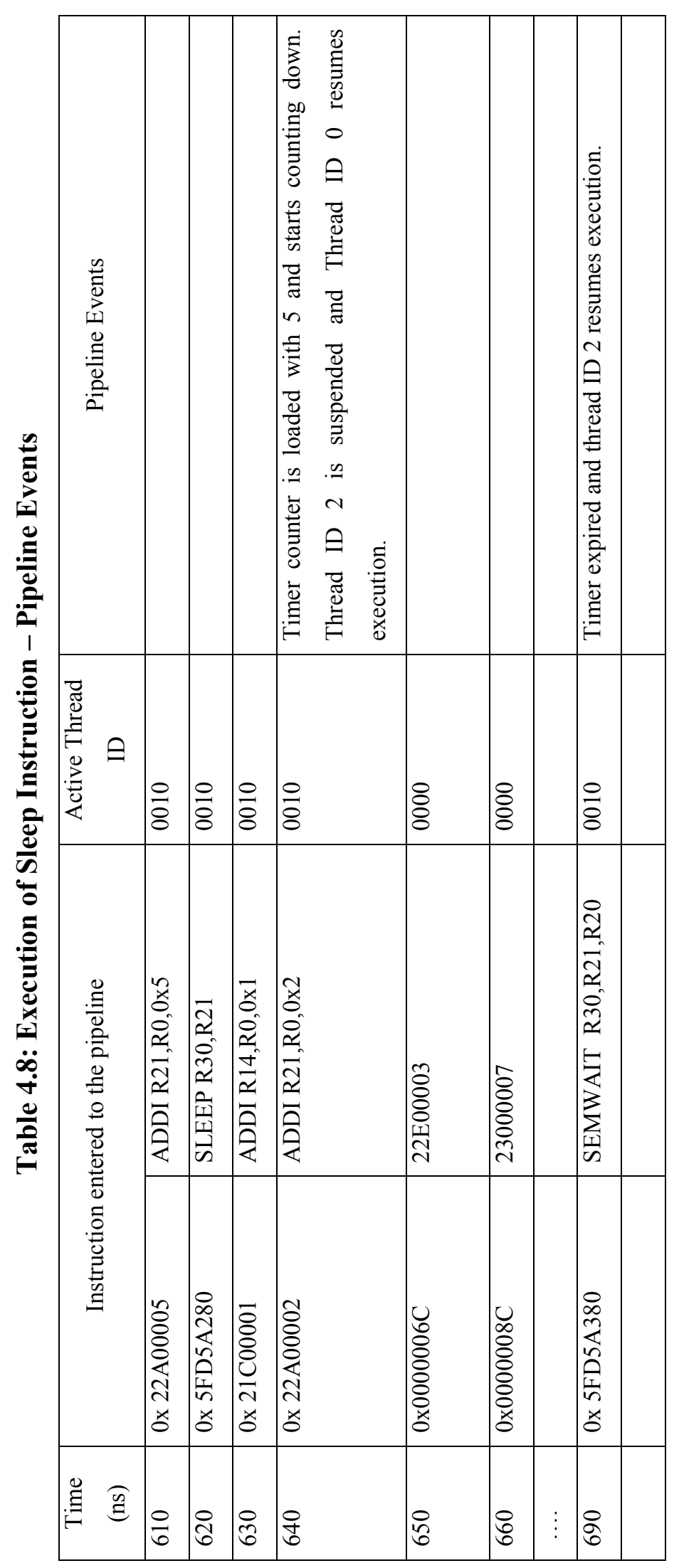

$\infty$ 


\subsubsection{Timing - Interrupt Service Threads}

The "IFORK" instruction creates an interrupt service thread. Two delay slots are used for this instruction. The "ICLEAR" instruction clears the interrupt and prepares the IST to service the next possible interrupt. The interrupt thread needs to execute the "ICLEAR" instruction once it services the interrupt. The "ICLEAR" instruction clears the interrupt bit and reloads the program counter of the IST with the starting address of the IST. Once the IST executes "ICLEAR", the idle thread resumes execution. This instruction has two delay slots.

Table 4.9 lists the assembly code used to create an IST with the priority value of 0xf and to clear the interrupt. Table 4.9 also lists the instructions at the starting address of the IST. Figure 4.9 illustrates the simulation results of IST creation .The simulation results in Figure 4.10 shows the activation of the interrupt service thread upon the occurrence of an external interrupt. As shown in the simulation results (provided that the IST related to the interrupt is the highest priority thread ready to run) the first instruction of the IST is scheduled during the next cycle. Figure 4.11 illustrates the simulation results related to interrupt clear. 
Table 4.9: A Program to Validate the IST Mechanism

\begin{tabular}{|c|c|c|c|c|}
\hline \# & $\begin{array}{l}\text { Memory } \\
\text { Address }\end{array}$ & Contents & Instruction & Description \\
\hline 1 & 0x00000050 & $0 \times 22800074$ & ADDI R20,R0,0x74 & $\begin{array}{l}\text { Starting address of the } \\
\text { new IST }\end{array}$ \\
\hline 2 & 0x00000054 & $0 \times 22 \mathrm{~A} 0000 \mathrm{~F}$ & ADDI R21,R0,0xF & Priority of the new IST \\
\hline 3 & 0x00000058 & 0x5FD5A100 & IFORK R30,R21,R20 & Create a new IST \\
\hline 4 & 0x0000005C & $0 \times 22 \mathrm{C} 00000$ & & Delay slot \\
\hline 5 & 0x00000060 & $0 \times 21800008$ & ADDI R12,R0,0x8 & Delay slot \\
\hline 6 & 0x00000064 & $0 \times 21 \mathrm{~A} 00002$ & ADDI R13,R0,0x2 & \multirow{3}{*}{ Idle loop } \\
\hline 7 & 0x00000068 & 0x058D6000 & RSUB R12,R13,R12 & \\
\hline 8 & 0x0000006C & 0xBC8CFFF8 & BGTI R12,-0x8 & \\
\hline \# & \multicolumn{4}{|c|}{ Interrupt Service Thread } \\
\hline 1 & 0x00000074 & $0 \times 21800008$ & ADDI R12,R0,0x8 & \multirow{5}{*}{ Service the interrupt. } \\
\hline 2 & $0 x 00000078$ & $0 \times 21 \mathrm{~A} 00002$ & ADDI R13,R0,0x2 & \\
\hline 3 & 0x0000007C & 0x058D6000 & RSUB R12,R13,R12 & \\
\hline 4 & $\ldots$ & & & \\
\hline 5 & $\ldots$ & & & \\
\hline \# & \multicolumn{4}{|c|}{ IST - Interrupt Clear } \\
\hline 1 & $0 \mathrm{x} 0000008 \mathrm{C}$ & $0 \times 22800074$ & ADDI R20,R0,0x74 & Starting address of the IST \\
\hline 2 & 0x00000088 & $0 \times 22 \mathrm{~A} 0000 \mathrm{~F}$ & ADDI R21,R0,0xF & Priority of the IST \\
\hline 3 & 0x0000008C & 0x 5FD5A200 & ICLEAR R30,R21,R20 & Clears the interrupt \\
\hline 4 & 0x00000090 & $0 \times 21 \mathrm{C} 00001$ & ADDI R14,R0,0x1 & Sleep delay slot \\
\hline 5 & 0x00000094 & 0x 22A00005 & ADDI R12,R0,0x8 & Sleep delay slot \\
\hline
\end{tabular}




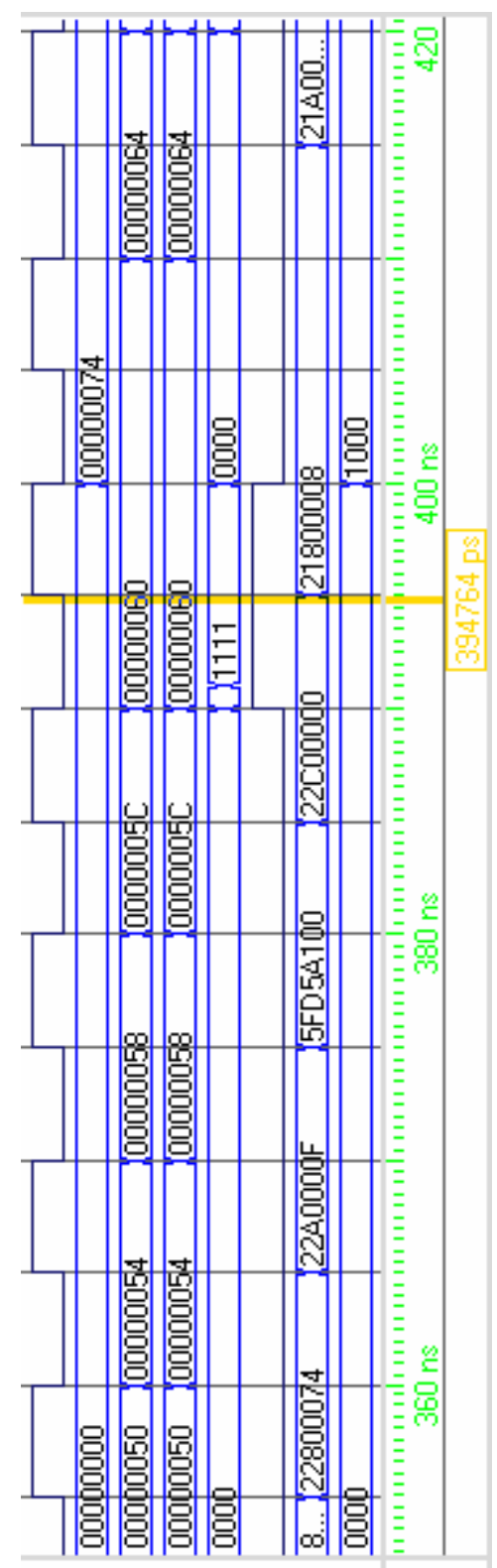

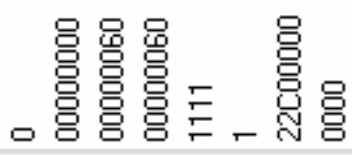

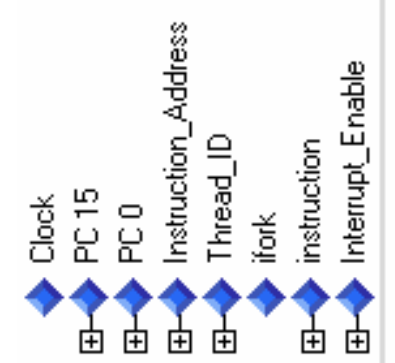

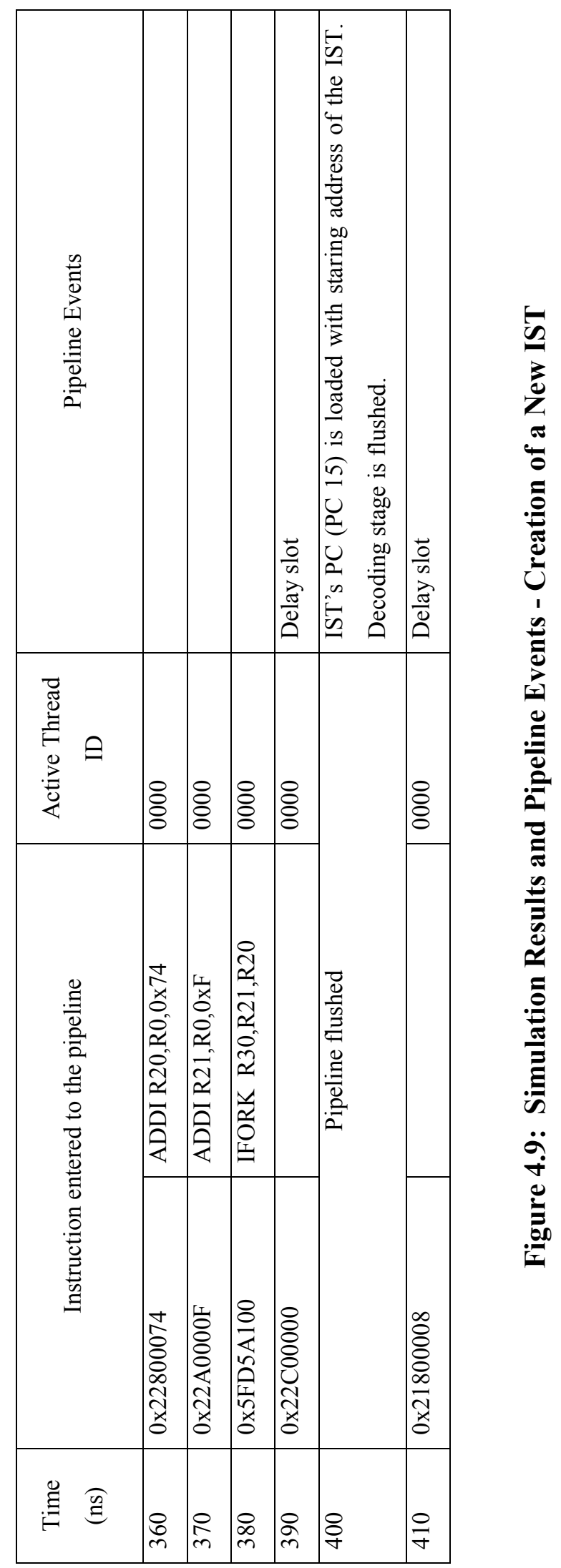



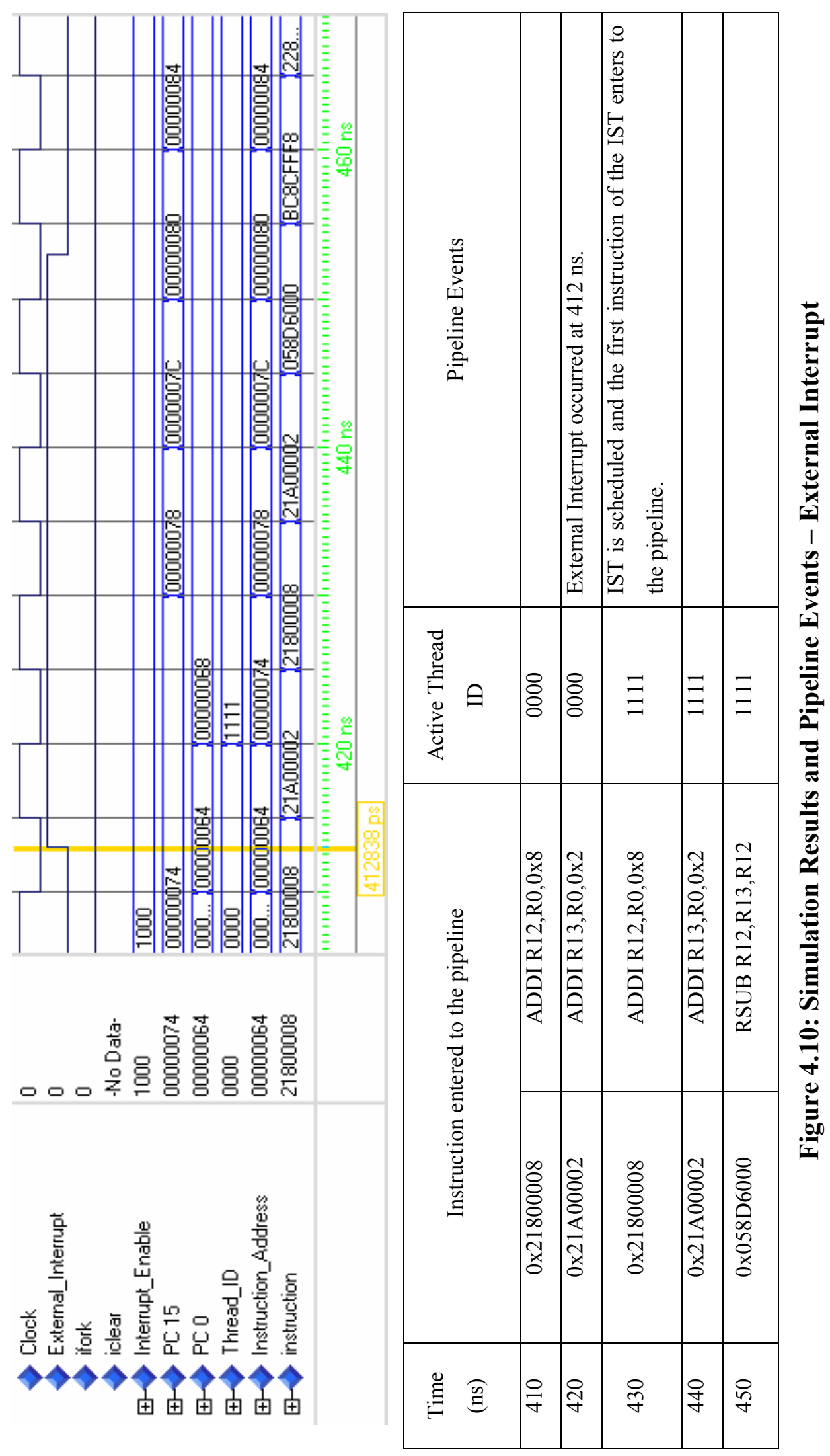

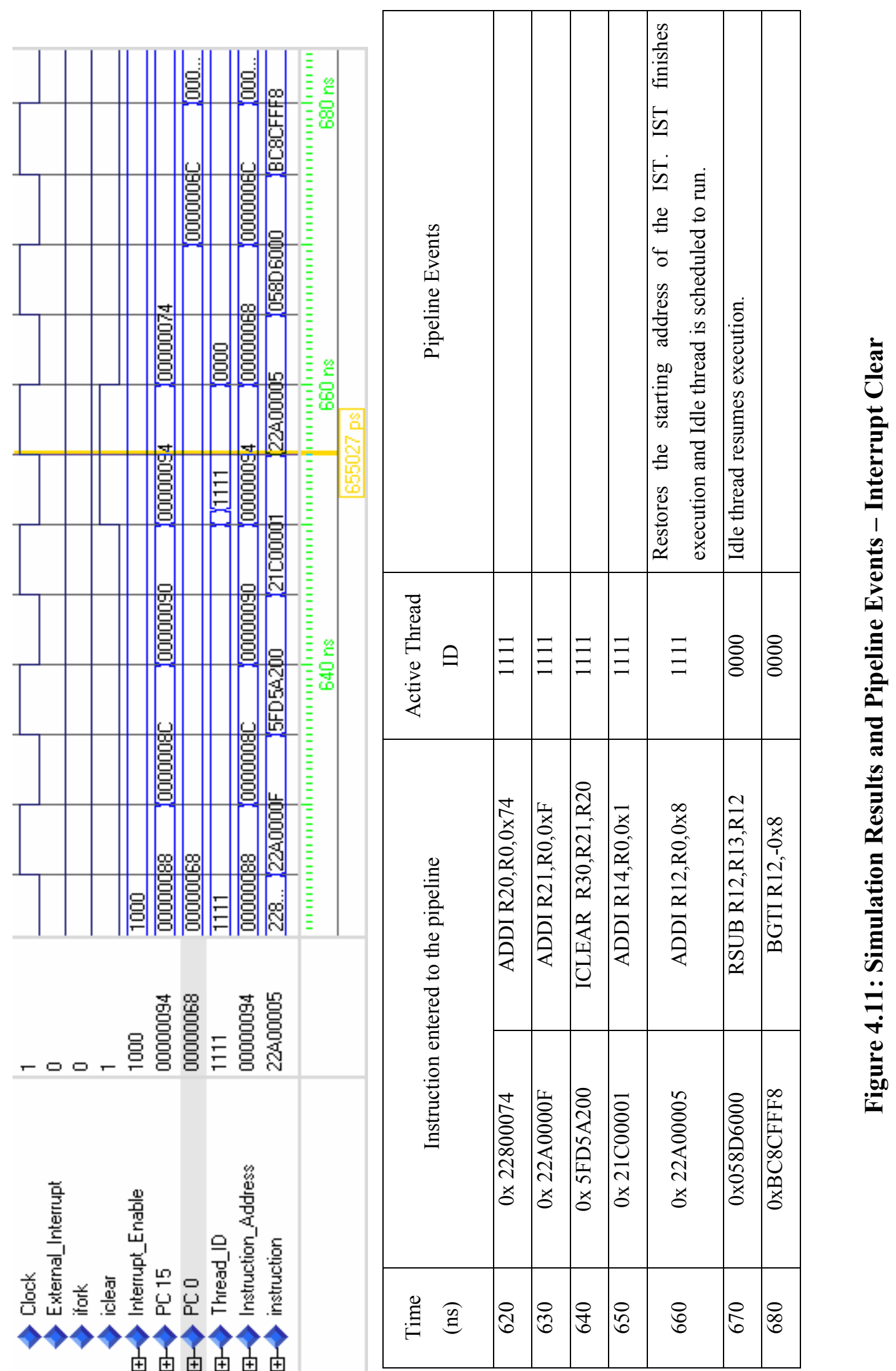


\subsubsection{Timing - Thread Synchronization Using Semaphores}

In order to validate the synchronization operations using the semaphore module, a simple program listed in Table 4.10 is used. The idle thread creates a new thread with priority value of two. The newly created thread initializes a semaphore to one and performs wait operation on the semaphore twice. This causes the thread to be blocked on the semaphore and idle thread to resume execution. Once resumed, idle thread performs a post operation on the semaphore which results in unblocking the TID 0x2. Figure 4.12, Figure 4.13 and Table 4.11 show the simulation results.

Table 4.10: A Program to Validate Semaphore Operations

\begin{tabular}{|l|l|l|l|l|}
\hline$\#$ & \multicolumn{1}{|c|}{$\begin{array}{c}\text { Memory } \\
\text { Address }\end{array}$} & \multicolumn{1}{|c|}{ Contents } & \multicolumn{1}{|c|}{ Instruction } & Description \\
\hline 1 & 0x0000008C & 0x 22800002 & ADDI R20,R0,0x2 & Semaphore ID = 0x02 \\
\hline 2 & 0x00000090 & 0x 22A00001 & ADDI R21,R0,0x1 & Semaphore value = 0x01 \\
\hline 3 & 0x00000094 & 0x 5FD5A300 & SEMINIT R30,R21,R20 & Creates the semaphore \\
\hline 4 & 0x00000098 & 0x 21C00001 & & \\
\hline 5 & 0x0000009C & 0x 22A00002 & ADDI R21,R0,0x2 & \\
\hline 6 & 0x000000A0 & 0x 5FD5A380 & SEMWAIT R30,R21,R20 & TID 0x2 waits on semaphore \\
\hline 7 & 0x000000A4 & 0x 22A00002 & ADDI R21,R0,0x1 & \\
\hline 8 & 0x000000A8 & 0x 22A00002 & ADDI R21,R0,0x1 & \\
\hline 9 & 0x000000AC & 0x 22800002 & ADDI R20,R0,0x2 & \\
\hline 10 & 0x000000B0 & 0x 22A00002 & ADDI R21,R0,0x2 & \\
\hline 11 & 0x000000B4 & 0x 5FD5A380 & SEMWAIT R30,R21,R20 & TID 0x2 waits on semaphore \\
\hline 12 & 0x000000B8 & 0x 22A00002 & ADDI R21,R0,0x2 & \\
\hline 13 & 0x000000BC & 0x 22A00003 & ADDI R21,R0,0x3 & \\
\hline 14 & 0x000000C0 & 0x 22A00004 & ADDI R21,R0,0x4 & \\
\hline 15 & 0x000000C4 & 0x 22A00005 & ADDI R21,R0,0x5 & \\
\hline Thread ID 0x00 & & \multicolumn{2}{|l|}{} \\
\hline 16 & 0x00000060 & 0x 22800002 & ADDI R20,R0,0x2 & \\
\hline 17 & 0x00000064 & 0x 22A00002 & ADDI R21,R0,0x2 & \\
\hline 18 & 0x00000068 & 0x 5FD5A400 & SEMPOST R30,R20 & Signals the semaphore \\
\hline 19 & 0x0000006C & 0x 22A00003 & ADDI R21,R0,0x3 & \\
\hline 20 & 0x00000070 & 0x 22A00005 & ADDI R21,R0,0x5 & \\
\hline 21 & 0x00000074 & 0x 22A00005 & ADDI R21,R0,0x5 & \\
\hline
\end{tabular}




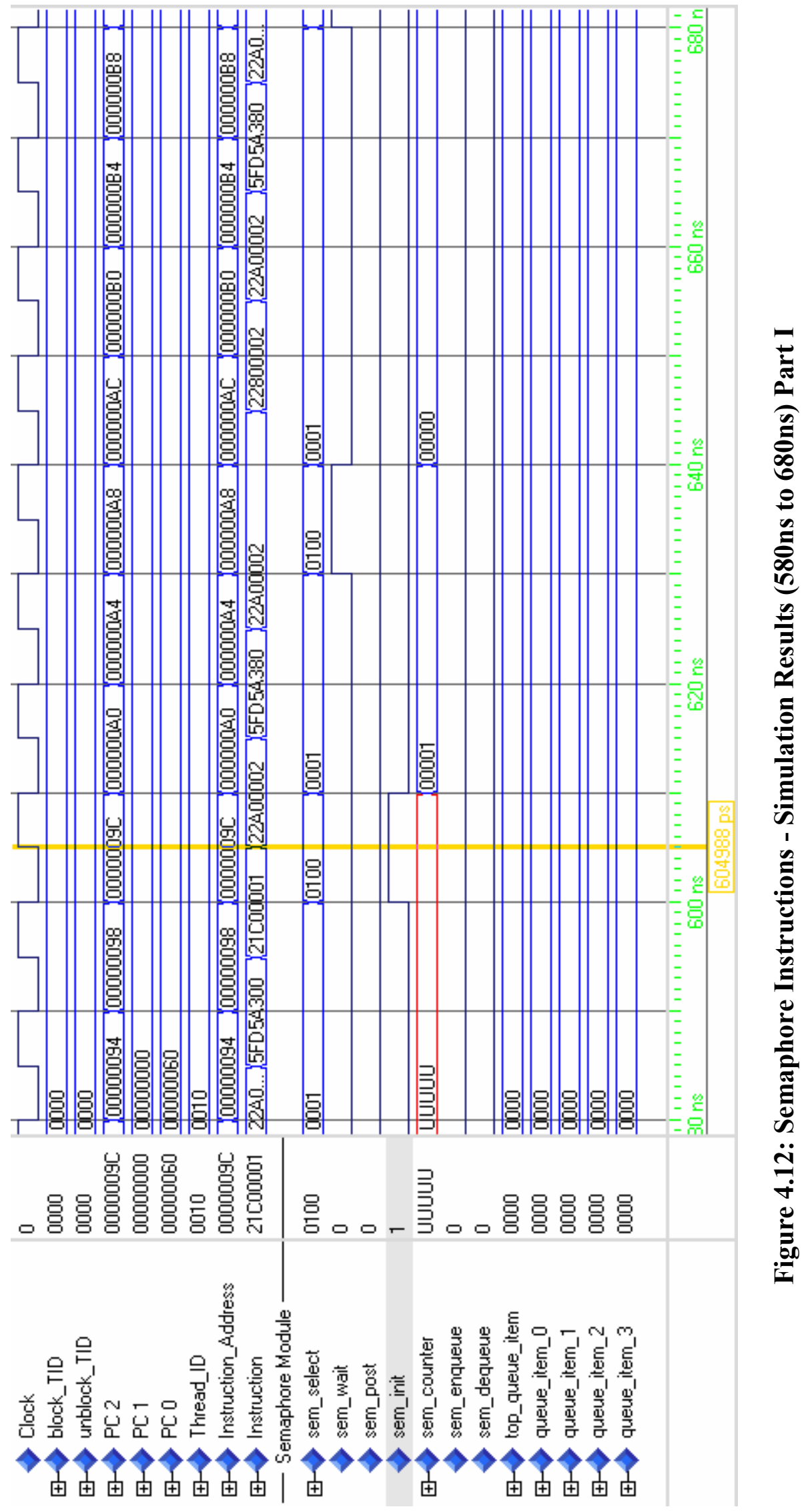




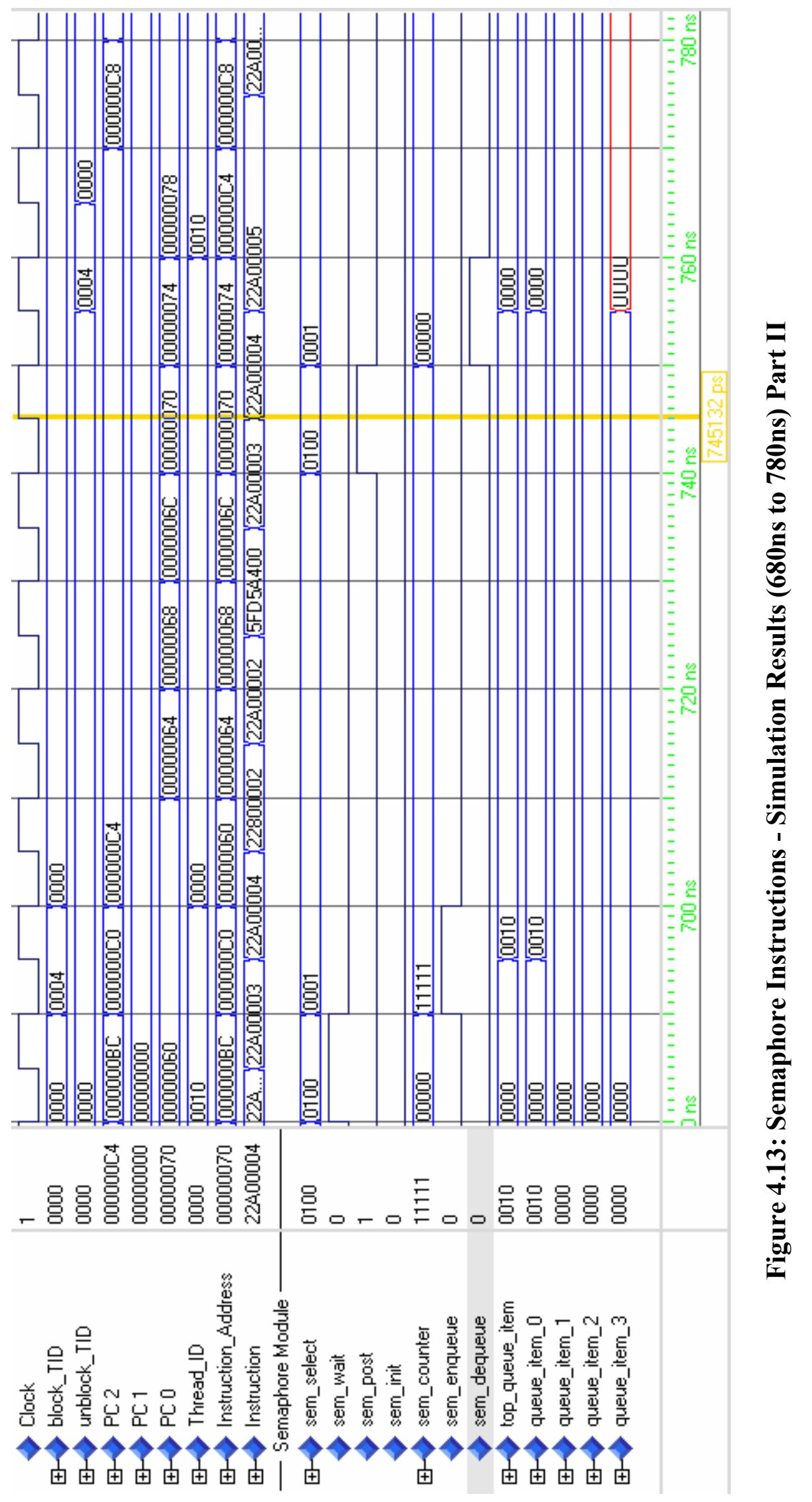




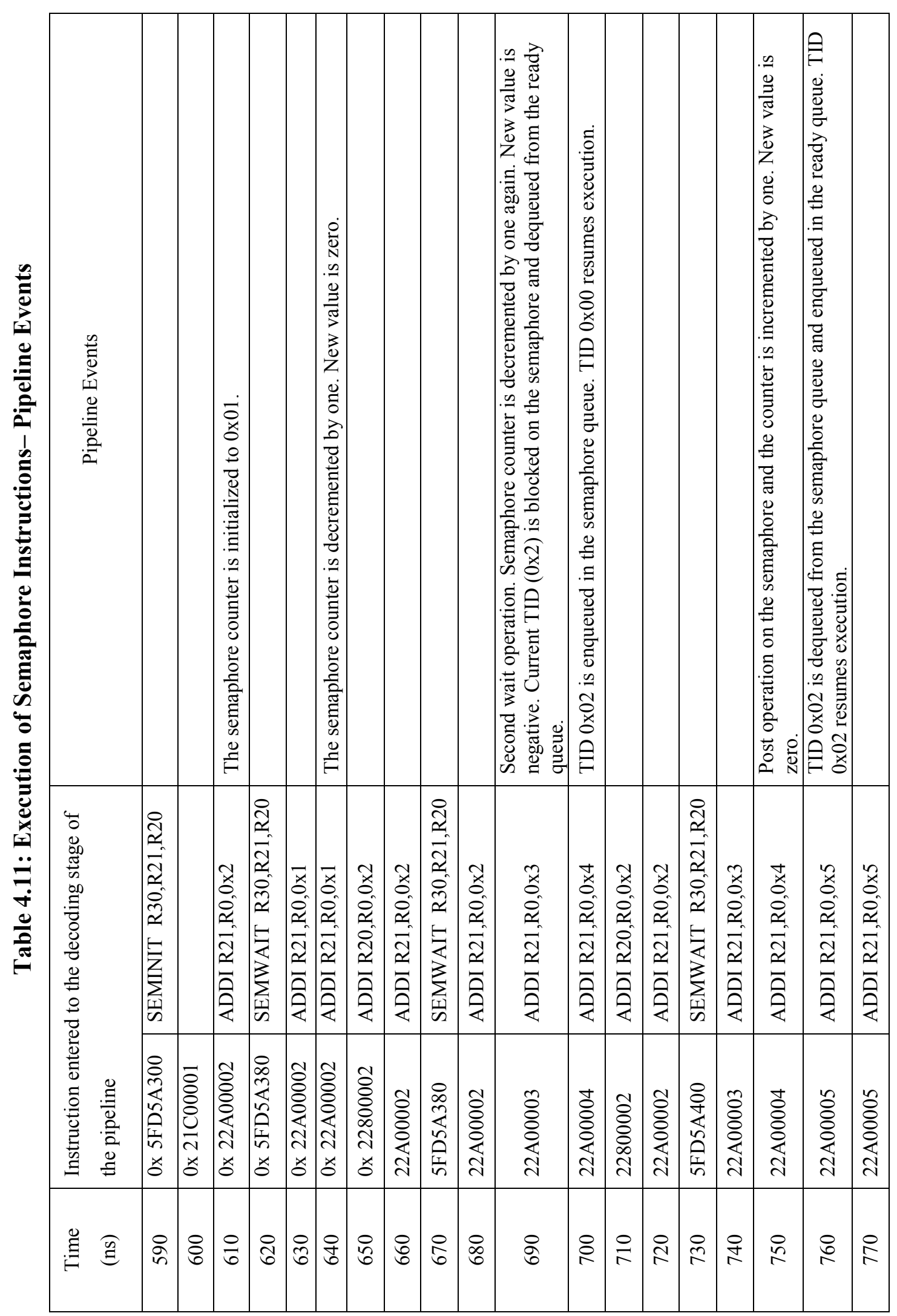




\subsection{Performance Comparison}

In order to evaluate the performance of RTBlaze, a comparison with software RTOSs needs to be carried out. Ronnholm [62] has evaluated three software RTOSs for Xilinx MicroBlaze processor. Xilkernal, Asterix and uClinux are selected for the comparison. The results related to those OSs are investigated in this section and compared with the RTBlaze simulation results.

uClinux [63] is a lightweight version of the standard Linux kernel [64], targeting microcontrollers. The main difference is that uClinux does not support virtual memory. The development is done by the standard GNU tool chain used for Linux. The scheduling model is the same as that of Linux and supports three scheduling policies: round robin, First-In First-Out (FIFO) and slandered time sharing.

Asterix [65] is a research grade open-source RTOS developed by the Malardalen Real-Time Research Center at Malardalen University. A separate configuration tool is used to configure the kernel parameters such as system clock resolution and TCB list. EDK compiler tools can be used for the Asterix based application software development. The kernel supports static priority preemptive scheduling as well as an event triggered mode of operation. The interrupt handling architecture has two levels which are the same as Xilkernal.

Xilkernal [39] is an open source small footprint robust software RTOS that comes with the Xilinx EDK tool chain. Xilkernal provides a POSIX like interface with the ability to handle semaphores, interrupts, mutexes and message queues. Modularity of the kernel allows configurability of these modules. As a result, the kernel can be tailored for the specific needs of the given application. Xilkernal supports both priority-based preemptive scheduling and round robin scheduling. The policy is global and needs to be initialized at configuration time of the kernel.

The SoPC used for the evaluation of the RTOSs are minimalist and only include the IP components required to implement minimalist Microblaze based SoPC running the RTOSs [62]. The SoPC system includes the following.

- $100 \mathrm{MH}$ system clock

- 64 MB DDR ram 
- 16 KB L1 I-cache

- $16 \mathrm{~KB}$ L1 D-cache

- OPB timer [66]

The software setup is also simple and Xilkernal and Asterix have only one task running (the idle task). uClinux have seven system tasks running. The evaluation is based on interrupt latency and task response time. Interrupt latency is the number of clock cycles the kernel takes to start executing the interrupt handling routing once an external interrupt occurs. The benchmarking is done by generating a periodic external interrupt using the OPB timer and reading the timer counter register at the beginning of the ISR to find out the cycles the kernel consumed in order to reach the ISR. The task response time, the time the kernel takes to reschedule the next highest priority task from the occurrence of an external interrupt, is also measured using a similar manner. This measurement is a fair estimation of the scheduler overhead.

\subsubsection{Comparison of the Performance of RTBlaze and Software RTOSs}

The simulation environment used to validate RTBlaze is almost similar to the setup used to evaluate the RTOSs for Microblaze. Ronnholm's [62] evaluation of RTOSs for MicroBlaze uses two configurations: cache enabled and cache disabled for benchmarks. RTBlaze is a cacheless CPU which targets real-time systems based on SoPC based systems. The program memory is supposed to be on chip block RAM which runs at full CPU speed. The memory access of RTBlaze is equivalent to the Microblaze system with cache enabled since all the benchmark code resides in the cache [62]. This makes the comparison between the Microblaze processor with cache enabled benchmark results [62] and RTBlaze simulation results reasonable. Table 4.12 summarizes the comparison results of the interrupt latency and task response time. 
Table 4.12: Latencies

\begin{tabular}{|c|c|c|}
\hline RTOS & $\begin{array}{c}\text { Interrupt Latency } \\
\text { Cycles(Time) }\end{array}$ & $\begin{array}{c}\text { Task Response Time } \\
\text { Cycles(Time) }\end{array}$ \\
\hline uClinux & $5630(56.3 \mathrm{uS})$ & $22441(224.4 \mathrm{uS})$ \\
\hline Xilkernal & $564(5.64 \mathrm{uS})$ & $2590(25.90 \mathrm{uS})$ \\
\hline Asterix & $494(4.94 \mathrm{uS})$ & $2508(25.08 \mathrm{uS})$ \\
\hline RTBlaze & $5(50 \mathrm{nS})$ & $5(50 \mathrm{nS})$ \\
\hline
\end{tabular}

As shown in the simulation results in Section 4.1, RTBlaze completely removes the RTOS overhead because of the hardware migration of the components of the RTOS. Upon an external interrupt, given that the IST is the highest priority ready thread, the IST is scheduled in the next CPU cycle. Obviously, RTBlaze has zero scheduler overhead, where the return from the interrupt service thread causes the next highest priority thread to be scheduled in the next CPU cycle.

\subsection{Size of the Kernel Footprint}

When it comes to RTOSs, the kernel size is a very important factor. RTBlaze does not need any software code for the kernel operation as it implements the primitive OS level functionality directly in hardware. Comparison results of the kernel footprints of the RTOSs are given in Table 4.13.

Table 4.13: Kernel Footprint Comparison

\begin{tabular}{|l|c|}
\hline \multicolumn{1}{|c|}{ RTOS } & Kernel Footprint \\
\hline uClinux & $1.759 \mathrm{MB}$ \\
\hline Asterix & $7.70 \mathrm{kB}$ \\
\hline Xilkernal (minimalist) & $19.97 \mathrm{kB}$ \\
\hline RTBlaze & $0 \mathrm{kB}$ \\
\hline
\end{tabular}




\subsection{Analysis of Area Overhead}

The chip utilization of FPGAs is measured using the number of FPGA logic resources being used. In order to analyze the area overhead, the LUT and flip flop count are usually used. Removal of OS overhead comes at the increased utilization of the chip area, and this overhead is measured using LUTs and flip flops. This section analyzes the chip area utilization of the real-time extensions in comparison with Microblaze processor and the multithreaded base processor.

Currently, the base processor implements a subset of the Microblaze processor instruction set. This includes the instruction set of a minimalist Microblaze processor. The configuration of the RTBlaze used for this comparison has the following features.

- 16 threads

- 4 interrupt service threads

- 15 timers

- 4 semaphores each with 4-element deep wait queue

Table 4.14 shows the comparison results of Microblaze, multithreaded base processor and RTBlaze.

Table 4.14: Resource Utilization Of RTOS Extensions

\begin{tabular}{|l|c|c|c|}
\hline & LUTs & Slice Flip Flops & Block RAMs \\
\hline Microblaze (Minimalist) & 975 & 485 & 0 \\
\hline Base processor & 674 & 309 & 0 \\
\hline RTBlaze & 1652 & 712 & 3 \\
\hline
\end{tabular}

Real-time extensions have resulted in almost doubling the area of the base processor. The resource usage of the base processor is lower than the Xilinx MicroBlaze processor with minimalist configuration. This is due to the fact that the prototype base processor does not implement all the bus interfaces that come with the Microblaze processor. Table 4.15 further analyzes the breakdown of the resource usage due to the RTOS extension. 
Table 4.15: Breakdown of Area Overhead Due to RTOS Extensions

\begin{tabular}{|l|l|l|}
\hline \multicolumn{1}{|c|}{ RTOS Component } & \multicolumn{1}{|c|}{ LUTs } & \multicolumn{1}{c|}{ Slice Flip Flops } \\
\hline $\begin{array}{l}\text { Scheduler and PC } \\
\text { counter bank }\end{array}$ & $15.5 \%$ & $4.9 \%$ \\
\hline Semaphore module & $41.6 \%$ & $27.7 \%$ \\
\hline Timer module & $39.7 \%$ & $67.4 \%$ \\
\hline Other & $3.2 \%$ & $0 \%$ \\
\hline
\end{tabular}

The timer module has the highest overall resource usage. The next highest resources usage is due to the addition of the semaphore module. The resource requirement of the scheduler is under $20 \%$. Other resources include the control unit modification in order to support RTOS instructions and the data path modifications due to the attachment of RTOS components. 


\section{Chapter 5}

\section{Conclusions and Future Work}

\subsection{Conclusions}

Real-time operating systems with conventional processors are widely used in the context of embedded computing. The overhead due to the software kernel contributes to the degradation the overall performance of RTOS based embedded systems. Previous research efforts focusing on migrating the RTOS functionality into hardware provide promising results. Multithreaded processors are a distinct area of research targeted to exploit thread level parallelism by interleaving multiple threads at the hardware level. The concept of multithreading can easily be extended to achieve a zero overhead context switching mechanism and fast interrupt service mechanism. Based on earlier research efforts, this thesis proposes a new multithreaded softcore processor, RTBlaze, with hardware RTOS support in order to eliminate the overhead due to software. 
The attachment of the hardware migrated RTOS components to a multithreaded processor gives various advantages over the IP based separate migration approach. Use of the multithreaded processor as the base processor gives the ability of zero overhead context switching with the ability of implementing zero latency ISTs. The tight coupling of a hardware migrated scheduler completely removes the scheduler overhead. The scheduler works cooperatively with the hardware implemented semaphore module in order to realize a blocking synchronization scheme with zero overhead. Timers are also implemented in hardware and completely remove the overhead and provide timing with very high resolution. Unlike implementations of RTOS as separate chips, tightly coupling the RTOS functionality to the processor pipeline reduces the interfacing glue logic and nondeterminism occurs in system calls due to nondeterministic bus arbitration schemes. Not only the zero overhead OS level functionality, but RTBlaze owns one key feature, the zero size kernel. This leaves enough room to have all the application code in the on-chip memory. Current FPGA technology integrates more and more on chip memory enabling multiple RTBlazes to work in parallel with no external memory and cache.

The Vertex family is targeted for the prototype implementation of RTBlaze. The processor runs at $119 \mathrm{MHz}$ of clock speed with hardware level support for 16 threads, 4 ISTs, 4 semaphores and 15 timers. RTBlaze outperforms conventional software RTOSs by providing interrupts and scheduling latencies as low as $50 \mathrm{~ns}$.

Migration and attachment of RTOS functionality to the processor substantially increase the area required to synthesis the RTBlaze processor. For the current prototype, the RTOS extension has almost doubled the base processor size. When it comes to the RTBlaze based SoPC systems, the RTBlaze processor reduces the area required to implement the system by reducing the number of IPs required. Multiple numbers of external interrupt lines eliminate the need of an external interrupt controller. EDK based SoPC uses timer IP to provide timer interrupt while RTBlaze eliminates this while providing high resolution timing. Apart from that, the area required to implement the cache controllers for both processors, the cache coherency mechanism and off chip memory controller is substantial. 
When it comes to the real application domains, fast interrupt service threads, a zero overhead synchronization mechanism with high resolution timers simplify the external interface design. For example, the sampling subsystem of the digital control systems can be easily and efficiently implemented using these resources. Unlike software based RTOSs, the high resolution timers with zero overhead interrupts make it possible to implement real-time digital controllers with very high sampling rates. Apart from that, the software solution to implement communication interfaces such as RS232, SPI, etc. results in reducing the number of IP cores required in SoPC based system.

\subsection{Future Work}

RTBlaze is a basic prototype level implementation and needs several improvements. Most critical improvements identified are as follows.

- Implementation and verification of the complete instruction set

- Implementation of bus interfaces such as OPB and PLB

- Implementation of exception handling threads

- Extension of the scheduler to support round robin scheduling policy

- Addition of a mechanism to address priority inversion

- Addition of configurability through a front-end configuration tool

- Extension of the GNU GCC backend to support RTOS instructions

The above improvements would allow seamless integration with EDK tool chain and design of complete SoPC systems using EDK library IPs. The delay slots can be filled with independent instructions from the thread by moving it down. This optimization can be automated by retargeting the GCC backend.

Further optimizations to the RTBlaze are also possible,

- Implement an EDF like scheduler

- More efficient mapping of FPGA resources and full structural level design of RTBlaze 
New scheduling algorithms can also be explored by combining conventional process scheduling algorithms with the cycle or block interleaved multithreading facility of the RTBlaze.

Multiprocessor systems with distributed hardware RTOS nodes can be built using an array of RTBlaze processors. EDK already has support for multiprocessor designs, so this can be easily designed using the Xilinx tool chain. To enable multiprocessor support, an inter-processor synchronization mechanism needs to be introduced to synchronize inter-processor level threads. 


\section{References}

[1] L. Lindh, "FASTHARD - A Fast Time Deterministic HARDware Based Realtime Kernel," in Proceedings of the Fourth Euromicro workshop on Real-Time Systems: IEEE, 1992, pp. 21 - 25, ISBN: 0-8186-2815-4.

[2] T. Nakano, A. Utama, M. Itabashi, A. Shiomi, and M. Imai, "Hardware implementation of a real-time operating system," in Proceedings of the 12th TRON Project International Symposium Washington, DC, USA: IEEE Computer Society 1995, pp. 34, ISBN:0-8186-7207-2.

[3] D. Niehaus, K. Ramamritham, J. A. Stankovic, G. Wallace, C. Weems, W. Burleson, and J. Ko, "The Spring scheduling co-processor: Design, use, and performance," in Proceedings of the Real-Time Systems Symposium Raleigh Durham, NC, USA: IEEE, 1993, pp. 106-111, ISBN: 0-8186-4480-X.

[4] P. Kohout, B. Ganesh, and B. Jacob, "Hardware support for real-time operating systems," in International Symposium on Systems Synthesis, Proceedings of the 1st IEEE/ACM/IFIP international conference on Hardware/software codesign and system synthesis Newport Beach, CA, USA: ACM, 2003, pp. 45 - 51, ISBN:1-58113-742-7.

[5] J. Hildebrandt, F. Golatowski, and D. Timmermann, "Scheduling coprocessor for enhanced least-laxity-first schedulingin hard real-time systems," in Proceedings of the 11th Euromicro Conference on Real-Time Systems York, UK: IEEE, 1999, pp. 208-215, ISBN: 0-7695-0240-7.

[6] S. Saez, J. Vila, A. Crespo, and A. Garcia, "A hardware scheduler for complex real-time systems," in Proceedings of the IEEE International Symposium on Industrial Electronics. ISIE Bled, Slovenia: IEEE, 1999, pp. 43-48, ISBN: 07803-5662-4.

[7] J. Hildebrandt and D. Timmermann, "An FPGA based Scheduling Coprocessor for Dynamic Priority Scheduling in Hard Real-Time Systems," in Field- 
Programmable Logic and Applications: The Roadmap to Reconfigurable Computing: SpringerLink, 2000, pp. 777-780, ISBN 978-3-540-67899-1.

[8] P. Kuacharoen, M. A. Shalan, and V. J. M. Iii, "A configurable hardware scheduler for real-time systems," in Proceedings of the International Conference on Engineering of Reconfigurable Systems and Algorithms: CSREA Press, 2003, pp. 96-101.

[9] L. Lindh and F. Stanischewski, "FASTCHART-Idea and Implementation," in Proceedings of the IEEE International Conference on Computer Design on VLSI in Computer \& Processor Washington, DC, USA: IEEE Computer Society, 1991, pp. 401-404, ISBN:0-8186-2270-9.

[10] T. Ungerer, B. Robič, and J. Šilc, "A survey of processors with explicit multithreading," in ACM Computing Surveys New York, NY, USA, 2003, pp. 29-63, ISSN:0360-0300.

[11] U. Brinkschulte, C. Krakowski, C. Kreuzinger, and T. Ungerer, Interrupt service threads - a new approach to handle multiple hard real-time events on a multithreaded microcontroller: RTSS WIP sessions, 1999.

[12] U. Brinkschulte, C. Krakowski, J. Kreuzinger, and T. Ungerer, "A Multithreaded Java Microcontroller for Thread-Oriented Real-Time Event Handling," in Proceedings of the International Conference on Parallel Architectures and Compilation Techniques Washington, DC, USA: IEEE Computer Society, 1999, pp. 34, ISBN:0-7695-0425-6.

[13] J. Kreuzinger, R. Marston, T. Ungerer, U. Brinkschulte, and C. krakowski, "The komodo project: thread-based event handling supported by a multithreaded java microcontroller," in 25th EUROMICRO Conference Milan, Italy, 1999, pp. 122128.

[14] M. Pfeffer, S. Uhrig, T. Ungerer, and U. Brinkschulte, "A Real-Time Java System on a Multithreaded Java Microcontroller," in Proceedings of the Fifth IEEE International Symposium on Object-Oriented Real-Time Distributed Computing: IEEE Computer Society, 2002, pp. 34, ISBN:0-7695-1558-4. 
[15] "MicroBlaze Processor Reference Guide, EDK 9.1i, Xilinx product specification," Xilinx, www.xilinx.com/support/documentation/sw_manuals/mb_ref_guide.pdf.

[16] "Virtex-II Pro and Virtex-II Pro X Platform FPGAs: Complete Data Sheet": Xilinx, www.xilinx.com/support/documentation/user_guides/ug012.pdf.

[17] "EDK Concepts, Tools, and Techniques, EDK 9.1i," Xilinx, EDK 9.1i.

[18] V. Asokan, Designing Multiprocessor Systems in Platform Studio: Xilinx, www.xilinx.com/support/documentation/white papers/wp262.pdf

[19] M. Hübner, K. Paulsson, and J. Becker, "Parallel and Flexible Multiprocessor System-On-Chip for Adaptive Automotive Applications based on Xilinx MicroBlaze Soft-Cores " in 19th IEEE International Parallel and Distributed Processing Symposium, 2005, pp. 149, ISBN:1530-2075.

[20] "Intel® PXA270 Processor : Data Sheet," intel, www.intel.com/design/embeddedpca/applicationsprocessors/302302.htm.

[21] "AMBA ${ }^{\mathrm{TM}}$ Specification, Rev 2.0," Arm, www.arm.com/products/solutions/AMBA_Spec.htm.

[22] "CoreConnect Bus Architecture, 32-, 64-, 128-bit core on-chip bus standard," IBM, http://www-

01.ibm.com/chips/techlib/techlib.nsf/literature/CoreConnect_Bus_Architecture.

[23] "WISHBONE System-On-Chip (SoC) Interconnection Architecture for Portable IP Cores," OpenCores, www.opencores.org/projects.cgi/web/wishbone/.

[24] "Processor Local Bus (PLB) v3.4 (v1.02a)": Xilinx, www.xilinx.com/support/documentation/ip_documentation/plb_v34.pdf, 2006.

[25] "On-Chip peripheral Bus V2.0 with OPB Arbiter": Xilinx, www.xilinx.com/support/documentation/ipembedprocess_coreconnect_opbbusstr uct.htm.

[26] "ISE In-Depth Tutorial": Xilinx, www.download.xilinx.com/direct/ise9 tutorials/ise9tut.pdf, 2007.

[27] Development System Reference Guide, Xilinx ISE 9.1i: Xilinx, www.toolbox.xilinx.com/docsan/xilinx9/books/docs/dev/dev.pdf. 
[28] R. Ernst, "Codesign of embedded systems: status and trends," IEEE Design \& Test of Computers, pp. 45-54, 1998.

[29] "Microprocessor Debug Module (MDM) (v2.00a)": Xilinx, www.china.xilinx.com/support/documentation/ip_documentation/mdm.pdf.

[30] M. Hübner and J. Becker, "Exploiting Dynamic and Partial Reconfiguration for FPGAs - Toolflow, Architecture and System Integration," in Proceedings of the 19th annual symposium on Integrated circuits and systems design: ACM, 2006, pp. 1-4, ISBN:1-59593-479-0.

[31] "Two Flows for Partial Reconfiguration: Module Based or Difference Based," Xilinx, www.xilinx.com/support/documentation/application_notes/xapp290.pdf.

[32] "Local Memory Bus (LMB) V1.0," Xilinx, www.china.xilinx.com/support/documentation/ip documentation/lmb.pdf.

[33] "LMB BRAM Interface Controller v2.00a," Xilinx, www.china.xilinx.com/support/documentation/ip documentation/lmb bram if c ntlr.pdf.

[34] "Block RAM (BRAM) Block," Xilinx, www.xilinx.com/support/documentation/ip documentation/bram block.pdf.

[35] "Fast Simplex Link (FSL) Bus v2.10a," Xilinx, www.china.xilinx.com/support/documentation/ip_documentation/fsl_v20.pdf.

[36] A. Silberschatz, P. B. Galvin, and G. Gagne, Operating System Concepts, 7th Edition ed., 2007.

[37] A. Mohammadi and S. G. Akl, "Scheduling Algorithms for Real-Time Systems," School of Computing Queen's University Kingston, Ontario Canada, www.cs.queensu.ca/home/ak1/techreports/scheduling.pdf2005.

[38] A. Burns, "Scheduling hard real-time systems: a review " Software Engineering, www.cse.ucsc.edu/ sbrandt/courses/Winter00/290S/burns.pdf 1991.

[39] "OS and Libraries Document Collection, EDK 9.1i, 2006," Xilinx, www.xilinx.com/support/documentation/sw_manuals/edk10_oslib_rm.pdf.

[40] J. L. Hennessy and D. A. P. A. C. Arpaci-Dusseau, Computer Architecture: A Quantitative Approach, 4th Edition ed., 2007. 
[41] A. El-Haj-Mahmoud and E. Rotenberg, "Safely exploiting multithreaded processors to tolerate memory latency in real-time systems," in Proceedings of the international conference on Compilers, architecture, and synthesis for embedded systems: IEEE, 2004, pp. 2-13, ISBN:1-58113-890-3.

[42] L. Lindh, J. Starner, and J. Furunas, "From Single to Multiprocessor Real-Time Kernals in Hardware," in Proceedings of the Real-Time Technology and Applications Symposium: IEEE, 1995, pp. 42, ISBN:0-8186-6980-2.

[43] "RealFast Intellectual Property," Vasteras,Sweden, www.realfast.se/sierra.

[44] T. Klevin, "Get RealFast RTOS with Xilinx FPGAs," Xcell Journal, pp. 8-12, 2003.

[45] J. A. Stankovic, D. Niehaus, and K. Ramamritham, " SpringNet: A Scalable Architecture for High Performance, Predictable, and Distributed Real-Time Computing, Technical Report," University of Massachusetts 1991.

[46] V. J.Mooney and D. M.Blough, " A Hardware-Software Real-Time Operating System Framework for SoCs," IEEE Design \& Test, pp. 44-51, ISSN: 07407475, 2002.

[47] D.-S. Sun, D. M. Blough, and V. J. Mooney, "Atalanta: A New Multiprocessor RTOS Kernel for System-on-a-Chip Applications, Technical Report," in Georgia Institute of Technology, 2002.

[48] A. Morton and W. M. Loucks, "A hardware/software kernel for system on chip designs," in Proceedings of the ACM symposium on Applied computing: ACM, 2004, pp. 869 - 875, ISBN:1-58113-812-1.

[49] "Intel ${ }^{\circledR} 64$ and IA-32 Architectures Software Developer's Manual Volume 3A: System Programming Guide, Part 1 ": Intel, www.download.intel.com/design/processor/manuals/253668.pdf.

[50] J.-D. Nicoud and A. M. Tyrrell, "The Transputer : T414 Instruction Set," IEEE Micro, pp. 60-75, ISSN:0272-1732, 1989.

[51] D. May, "Software Defined Silicon," in Bristol University and XMOS, www.XMOS.com.

[52] G. Hoover, F. Brewer, and T. Sherwood, "A case study of multi-threading in the embedded space," in Proceedings of the International Conference on Compilers, 
Architecture and Synthesis for Embedded Systems: ACM, 2006, pp. 357-367, ISBN:1-59593-543-6.

[53] K. Tanaka, "PRESTOR-1: A Processor Extending Multithreaded Architecture," in Innovative Architecture for Future Generation High-Performance Processors and Systems: IEEE, 2005, pp. 8, ISSN: 1527-1366, ISBN: 0-7695-2483-4.

[54] K. Tanaka, "Real-Time Operating System Kernel for Multithreaded Processor," in Proceedings of the International Workshop on Innovative Architecture for Future Generation High Performance Processors and Systems, IEEE: IEEE Computer Society, 2006, pp. 91-100.

[55] M. Schoeberl, "A Java processor architecture for embedded real-time systems," Journal of Systems Architecture: the EUROMICRO Journal, pp. 265-286, ISSN:1383-7621, 2003.

[56] "Xilkernel, EDK 9.1i, Xilinx product specification," Xilinx, EDK 9.1i.

[57] P. Glover, "Using and Creating Interrupt-Based Systems," Application Note: Embedded Hardware Systems, Xilinx, www.xilinx.com/support/documentation/application_notes/xapp778.pdf

[58] "Using Block RAM in Spartan-3 Generation FPGAs," Application Note, Xilinx, www.xilinx.com/support/documentation/application_notes/xapp463.pdf.

[59] J. P. Bradford and S. Abraham, "Efficient Synchronization for Multithreaded Processors," http://citeseerx.ist.psu.edu/viewdoc/summary?doi=10.1.1.56.6592.

[60] B. K. Kim and K. G. Shin, "Scalable hardware earliest-deadline-first scheduler for ATM switching networks," in 18th IEEE Real-Time Systems Symposium (RTSS '97), 1997, pp. 210, ISBN:0-8186-8268-X.

[61] B. M. Al-Hashimi, System-on-Chip: Next Generation Electronics (Circuits, Devices and Systems) 2006.

[62] A. Ronnholm, "Evaluation of Real-Time Operating Systems for Xilinx MicroBlaze CPU, Thesis," in Computer Science and Electronics: Malardalens University, 2006.

[63] "Embedded Linux/Microcontroller Project," http://www.uclinux.org/.

[64] D.P.Bovet and M. Cesati, Understanding the LINUX KERNAL: O'Reilly, 2003. 
[65] H. Thane, A. Pettersson, and D. Sundmark, "The asterix real-time kernel," in 13th EUROMICRO International Conference on Realtime Systems, Industrial Session Technical University of Delft, Delft, The Netherla: IEEE Computer Society, June 2001.

[66] "OPB Timer/Counter (v1.00b)," Product Specification, 2006, Xilinx, www.xilinx.com/support/documentation/ipembedprocess peripheralother opbti mercounter.htm. 